\title{
Marine-Derived Penicillium Species as Producers of Cytotoxic Metabolites
}

\author{
Sen Liu ${ }^{1}$ (D), Mingzhi Su ${ }^{1}$, Shao-Jiang Song ${ }^{2}$ and Jee H. Jung ${ }^{1, *}$ \\ 1 College of Pharmacy, Pusan National University, Busan 609-735, Korea; 1s691392008@sina.com (S.L.); \\ sumingzhi0310@gmail.com (M.S.) \\ 2 Department of Natural Products Chemistry, Shenyang Pharmaceutical University, Shenyang 110016, China; \\ songsj99@163.com \\ * Correspondence: jhjung@pusam.ac.kr; Tel.: +82-51-510-2803; Fax: +82-51-513-6754
}

Received: 10 August 2017; Accepted: 9 October 2017; Published: 24 October 2017

\begin{abstract}
Since the discovery of penicillin, Penicillium has become one of the most attractive fungal genera for the production of bioactive molecules. Marine-derived Penicillium has provided numerous excellent pharmaceutical leads over the past decades. In this review, we focused on the cytotoxic metabolites * ( Cytotoxic potency was referred to five different levels in this review, extraordinary $\left(\mathrm{IC}_{50} / \mathrm{LD}_{50}:<1 \mu \mathrm{M}\right.$ or $\left.0.5 \mu \mathrm{g} / \mathrm{mL}\right)$; significant $\left(\mathrm{IC}_{50} / \mathrm{LD}_{50}: 1 \sim 10 \mu \mathrm{M}\right.$ or $\left.0.5 \sim 5 \mu \mathrm{g} / \mathrm{mL}\right)$; moderate $\left(\mathrm{IC}_{50} / \mathrm{LD}_{50}: 10 \sim 30 \mu \mathrm{M}\right.$ or $5 \sim 15 \mu \mathrm{g} / \mathrm{mL}$ ); mild $\left(\mathrm{IC}_{50} / \mathrm{LD}_{50}: 30 \sim 50 \mu \mathrm{M}\right.$ or $\left.15 \sim 25 \mu \mathrm{g} / \mathrm{mL}\right)$; weak ( $\mathrm{IC}_{50} / \mathrm{LD}_{50}$ : 50 100 $\mu \mathrm{M}$ or $25 \sim 50 \mu \mathrm{g} / \mathrm{mL}$ ). The comparative potencies of positive controls were referred when they were available). produced by marine-derived Penicillium species, and on their cytotoxicity mechanisms, biosyntheses, and chemical syntheses.
\end{abstract}

Keywords: marine-derived Penicillium; natural products; cytotoxic metabolites; biosynthesis

\section{Introduction}

The oceans, which occupy more than $70 \%$ of the earth's surface, undoubtedly support vast habitats and serve as prolific resources of various living organisms. Compared to terrestrial organisms, marine organisms often produce highly potent metabolites with unique structures to enable them to adapt to extremely challenging environments [1]. Developments and improvements made in biotechnology have led to a new era of bioprospecting for new marine products. Revolutionary target screening methods have improved the efficiency of drug discovery. In addition, leading edge genomics of biological symbiosis offer more opportunities to discover drug candidates and precursors. Marine endozoic microorganisms represent a new frontier in the discovery of pharmaceutical agents [2]. In particular, marine-derived fungi are excellent producers of biologically active secondary metabolites. Since the isolation of the broad-spectrum antibiotic, cephalosporin $C$ from the marine-derived fungus Acremonium chrysogenum, thousands of bioactive metabolites have been discovered and evaluated [3].

Cancer is the second leading cause of death. Lung, prostate, colorectal, and digestive tract cancer are commonly encountered in males, whereas breast, lung, and cervical cancer are the major causes of female death. Marine microorganisms produce limited amounts of highly efficient toxic substances to protect their hosts from enemies, and these substances have been investigated as potential anticancer drug precursors. In particular, marine-derived Penicillium species represent a major source of cytotoxic metabolites. In this review, we list all cytotoxic or antitumor secondary metabolites isolated from marine-derived Penicillium species and classify them into distinct chemical groups. In addition, we summarize the cytotoxicity mechanisms and proposed biosyntheses of these metabolites. Overall, more than 200 natural products and their synthetic analogues are included in this review. 


\section{Alkaloids}

Cytochalasan alkaloids, characterized by a highly substituted perhydoisoindol-1-one fused to a macrocyclic ring, have been shown to possess potential cytotoxicity against diverse tumor cell lines [4,5]. Penochalasins, chaetoglobosins, and cytoglobosins are common classes of cytochalasan alkaloids. A series of cytochalasans, penochalasins A-J (1-10), chaetoglobosins A, C, E-G, $\mathrm{O}$ (11-16), and cytoglobosin C (17) (Figure 1) were isolated from the mangrove endophytic fungus P. chrysogenum [6] and from the marine alga Enteromorpha intestinalis [7,8]. Penochalasins A-H (1-8) and chaetoglobosins $\mathrm{A}, \mathrm{F}, \mathrm{O}(\mathbf{1 1}, \mathbf{1 4}, \mathbf{1 6})$ exhibited significant cytotoxic activity $\left(\mathrm{ED}_{50}=0.4,0.3,0.5\right.$, $3.2,2.1,1.8,1.9,2.8,0.6,0.9$, and $2.4 \mu \mathrm{g} / \mathrm{mL}$, respectively) against P388 lymphocytic leukemia cells. Moreover, chaetoglobosin A (11) reportedly induced apoptosis of chronic lymphocytic leukemia (CLL) cells by targeting the cytoskeleton. The underlying mechanisms involve the induction of cell-cycle arrest and the inhibition of membrane ruffling and cell migration; therefore, it was proposed as a novel drug for CLL [9]. Penochalasin I (9) exhibited significant cytotoxic activities against MDA-MB-435 (human breast cancer cell line) and SGC-7901 (human gastric cancer cell line) with $\mathrm{IC}_{50}$ values of $\sim 7 \mu \mathrm{M}$. Cytoglobosin C (17) showed potential cytotoxicity against both SGC-7901 and A549 (human lung adenocarcinoma) with $\mathrm{IC}_{50}$ values of 3-8 $\mu \mathrm{M}$. Other cytochalasans, penochalasin $\mathrm{J}(\mathbf{1 0})$, chaetoglobosins C, E (12, 13), and chaetoglobosin G (15) showed moderate cytotoxicity against MDA-MB-435, SGC-7901, and A549 with $\mathrm{IC}_{50}$ values in the range of $10-40 \mu \mathrm{M}$ (epirubicin was used as a positive control with $\mathrm{IC}_{50}$ values of $\left.0.3 \sim 0.6 \mu \mathrm{M}\right)$. A recent biosynthetic analysis showed that the fungal PKS-NRPS hybrid synthase, CheA, plays an essential role in cytochalasan formation [10].

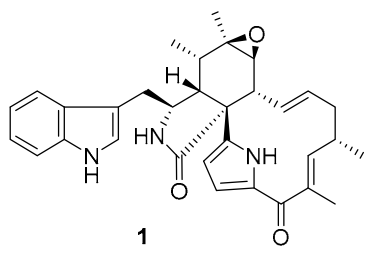

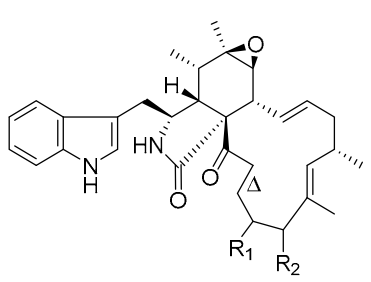

5: $\mathrm{R}_{1}==\mathrm{O}, \mathrm{R}_{2}=\alpha \mathrm{OH}, \Delta$ saturated 6: $\mathrm{R}_{1}==\mathrm{O}, \mathrm{R}_{2}=\mathrm{BOH}, \Delta$ saturated 11: $\mathrm{R}_{1}==\mathrm{O}, \mathrm{R}_{2}=\mathrm{BOH}, \Delta$ unsaturated 12: $R_{1}=R_{2}==0 \Delta$ saturated 14: $\mathrm{R}_{1}=\beta \mathrm{OH}, \mathrm{R}_{2}==\mathrm{O} \Delta$ saturated

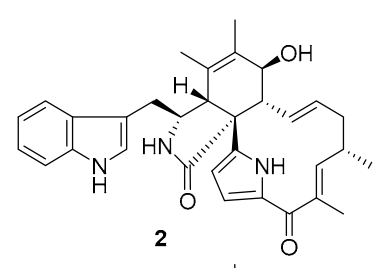

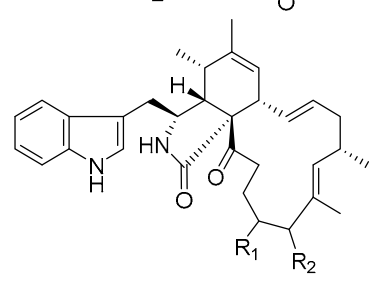

7: $\mathrm{R}_{1}==\mathrm{O}, \mathrm{R}_{2}=\mathrm{BOH}$ $9: R_{1}==O, R_{2}=H$

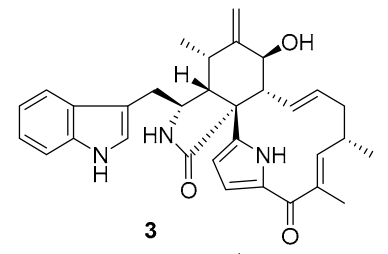

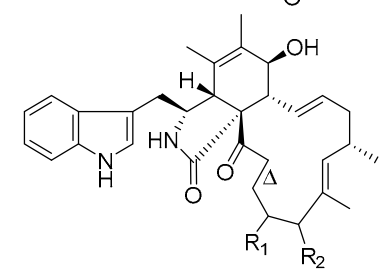

8: $\mathrm{R}_{1}==\mathrm{O}, \mathrm{R}_{2}=\alpha \mathrm{OH} \Delta$ saturated 13: $\mathrm{R}_{1}=\beta \mathrm{OH}, \mathrm{R}_{2}==\mathrm{O} \Delta$ saturated 15: $R_{1}=R_{2}==O \Delta$ saturated 16: $R_{1}==\mathrm{O}, \mathrm{R}_{2}=\beta \mathrm{OH} \Delta$ saturated 17: $R_{1}=\beta O H, R_{2}=\beta O H \Delta$ unsaturated

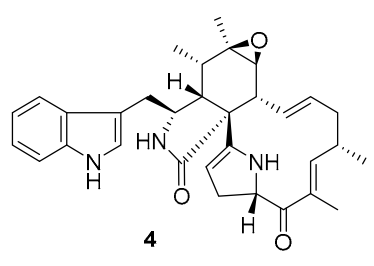

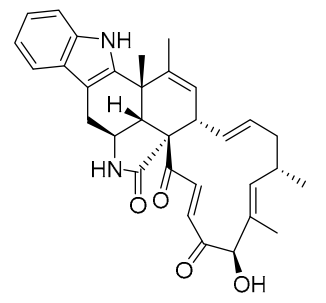

10

Figure 1. Chemical structures of compounds 1-17.

Gliotoxin induces cellular immunosuppression and apoptosis [11], and its analogues are disulfur or polysulfur-containing mycotoxins that belong to a class of naturally occurring epipolythio piperazines (ETP). In 2012, the marine fungus Penicillium sp. JMF034, which was isolated from a deep sea sediment in Japan, was found to produce seven gliotoxin-related compounds, (bis(dethio)-10a-methylthio-3a-deoxy-3,3a-didehydrogliotoxin (18), 6-deoxy-5a,6-dide hydrogliotoxin (19), bis(dethio) bis(methylthio)gliotoxin (20), bis(dethio)bis(methylthio)-5a,6-dide hydrogliotoxin (21), 5a,6-dide hydrogliotoxin (22), gliotoxin (23), and gliotoxin G (24) (Figure 2) [12], which potently killed P388 murine leukemia cells $\left(\mathrm{IC}_{50}=3.4,0.058,0.11,0.11,0.056,0.024\right.$, and $0.020 \mu \mathrm{M}$, respectively). Because of their extraordinary cytotoxicity, gliotoxin analogues are considered as antitumor leads [13]. Dimeric ETPs were reported to inhibit histone methyltransferase (HMT); in addition, compounds (22-24) with disulfide or tetrasulfide bonds showed significant inhibitory 
activities against HMT G9a $\left(\mathrm{IC}_{50}=2.6,6.4\right.$, and $2.1 \mu \mathrm{M}$, respectively) rather than HMT SET7/9 $\left(\mathrm{IC}_{50}>100 \mu \mathrm{M}\right)$. Gliotoxin $\mathrm{G}(24)$, isolated from the mangrove endophytic fungus P. brocae MA-231, was potently active against cisplatin-sensitive and resistant human ovarian cancer cell lines, A2780 and A2780 CisR, with IC $_{50}$ values of 664 and $661 \mathrm{nM}$, respectively (cisplatin was used as a positive control with $\mathrm{IC}_{50}$ values of 1.67 and $12.63 \mu \mathrm{M}$, respectively) [14]. Compound 24 may be used as an anti-ovarian cancer agent, even in patients who are resistant to platinum compounds. Plausible hypotheses for the biosyntheses of ETPs have been previously reviewed [15].

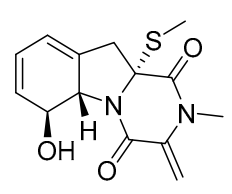

18

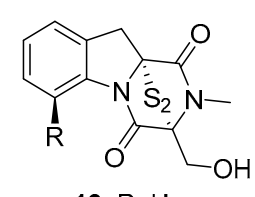

19: $\mathrm{R}=\mathrm{H}$ 20: $\mathrm{R}=\mathrm{OH}$

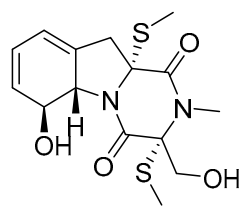

21

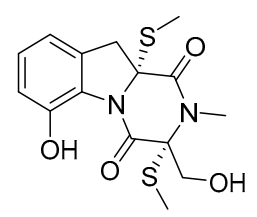

22

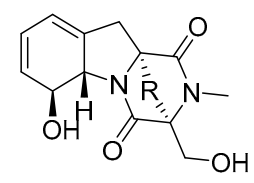

23: $R=S_{2}$

24: $\mathrm{R}=\mathrm{S}_{4}$

Figure 2. Chemical structures of compounds 18-24.

Four new cytotoxic bisthiodiketopiperazines (brocazines A-F) (25-30) (Figure 3), which share molecular similarities with gliotoxin, were isolated from a fungal strain of P. brocae MA-231, collected from the marine mangrove Avicennia marina [16]. Their cytotoxicity was investigated in human prostate cancer (DU145), human cervical carcinoma (Hela), human hepatoma (HepG2), human breast carcinoma (MCF-7), human large-cell lung carcinoma (NCI-H460), SGC-790, human pancreatic cancer (SW1990), human colon carcinoma (SW480), and human glioma (U251) cell lines. Brocazines A, B, E, and F (25, 26, 29, and 30) exhibited significant cytotoxic effects against most of the cell lines tested with $\mathrm{IC}_{50}$ values in the range of $0.89-9 \mu \mathrm{M}$ (paclitaxel, cisplatin, cefitinib, doxorubicin, and gemcitabine were used as positive controls with $\mathrm{IC}_{50}$ values of $\left.1 \sim 11 \mu \mathrm{M}\right)$. In contrast, brocazines $\mathrm{C}$ and $\mathrm{D}$ (27 and 28), which lack the $\alpha, \beta$ unsaturated ketone group, had much lower cytotoxicity $\left(\mathrm{IC}_{50}>20 \mu \mathrm{M}\right)$, which suggests that the conjugated ketone system is crucial to the cytotoxic properties of bisthiodiketopiperazine analogues.

Two bisthiodiketopiperazines, pretrichodermamide C (31) and $N$-methylpretrichodermamide B (32) (also called adametizine B and A, respectively) (Figure 4), were isolated from a marine sponge-derived fungus (P. adametzioides AS-53) [17], a hyper saline lake-derived Penicillium sp. [18], and a marine algicolous fungus (Penicillium sp. KMM4672) [19]. All three studies showed that compound 32, which contains chlorine, exhibited significant cytotoxicity, wherein it reduced the viability of L5178Y mouse lymphoma cells, human prostate cancer 22Rv1 cells, PC-3 cells, LNCaP cells, and brine shrimps ( $\mathrm{IC}_{50}=2,0.51,5.11,1.76$, and $4.8 \mu \mathrm{M}$, respectively; while kahalalide $\mathrm{F}$, docetaxel, and colchicine were employed as positive controls with $\mathrm{IC}_{50}$ values of 4.3, 0.013, 0.015, 0.004 , and $8.1 \mu \mathrm{M}$, respectively). Furthermore, it was found active in hormone-resistant 22Rv1 cells at nanomolar concentrations. In contrast, metabolite $\mathbf{3 1}$ was completely inactive in all bioassays with $\mathrm{IC}_{50}$ values $>100 \mu \mathrm{M}$. This remarkable difference in activity indicates that the halogen atom might improve the activity of the metabolite. 


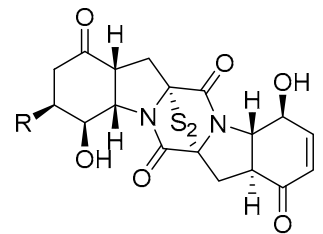

25: $\mathrm{R}=\mathrm{OCH}_{3}$ 26: $\mathrm{R}=\mathrm{H}$

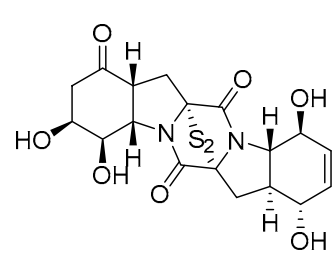

27

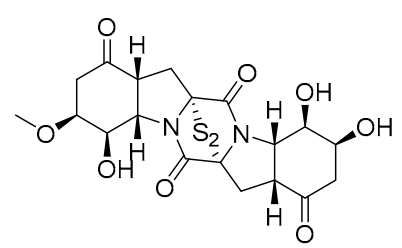

28

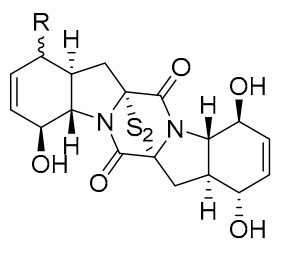

29: $\mathrm{R}=\alpha \mathrm{OH}$ 30: $R==0$

Figure 3. Chemical structures of compounds 25-30.

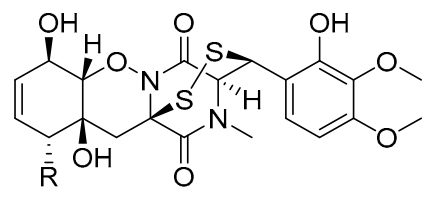

31: $\mathrm{R}=\mathrm{OH}$

32: $\mathrm{R}=\mathrm{Cl}$

Figure 4. Chemical structures of compounds 31-32.

Roquefortine C (33) (Figure 5) is a potential neurotoxin that can activate P-glycoprotein and simultaneously inhibit P450-3A and other hemoproteins [20]. Roquefortine and meleagrin (38) analogues are considered biogenetically interrelated mycotoxins with promising cytotoxicity [21]. Recently, a series of roquefortine derivatives, roquefortines F-I (34-37), and meleagrin analogues, meleagrins B-E (39-42), were isolated from the deep ocean sediment-derived fungus Penicillium sp. [22], and most of these compounds (34, 35, and 39-42) were active against A549, HL-60 (human promyelocytic leukemia), BEL-7402 (human hepatoma), and MOLT-4 (human acute T lymphoblastic leukemia) cancer cell lines. Meleagrin B (39) was the most cytotoxic against these four cell lines with $\mathrm{IC}_{50}$ values in the range of $1.5-7 \mu \mathrm{M}$; the other compounds had $\mathrm{IC}_{50}$ values in the range of $4-50 \mu \mathrm{M}$. Meleagrin (38) was also isolated from a deep sea sediment-derived fungus, P. commune SD-118, and was found to be cytotoxic in HepG2, NCI-H460, Hela, MDA-MB-231 (human breast cancer cells), and DU145 human cancer cell lines $\left(\mathrm{IC}_{50}=12,22,20,11\right.$, and $5 \mu \mathrm{g} / \mathrm{mL}$, respectively; while fluorouracil was employed as a positive control with $\mathrm{IC}_{50}$ values of $14,1,14,8$, and $0.4 \mu \mathrm{g} / \mathrm{mL}$, respectively) [23].

Penicimutanins A,B (43-45) and fructigenine A (46) (Figure 6) are structurally similar to roquefortines, and were first isolated from diethyl sulfate- or gentamicin-induced mutants of the marine-derived fungus P. purpurogenum G59 [24,25]. Mutation-based approaches can activate silent fungal gene clusters and afford more potent metabolites with unique structures. Compounds 44 and $\mathbf{4 5}$ are mutant cytotoxic products that showed potent activities against five human cancer cell lines: K562 (human chronic myelogenous leukemia), HL-60, Hela, BGC-823 (human gastric adenocarcinoma), and MCF-7 ( $\mathrm{IC}_{50}$ values were 5-11 $\mu \mathrm{M}$ for 44 and 8-20 $\mu \mathrm{M}$ for 45 ). Compounds 43 and 46 also inhibited the proliferation of these cell lines (Inhibition Rate (IR) $\%=22.6$ and 20.8 (K562); 17.9 and 55.3 (HeLa); and 26.5 and $65.6 \%$ (MCF-7) at $100 \mu \mathrm{g} / \mathrm{mL}$, respectively; while 5-fluorouracil was employed as a positive control with $\mathrm{IR} \%$ of $48.5,37.4$, and $47.4 \mu \mathrm{g} / \mathrm{mL}$ at $100 \mu \mathrm{g} / \mathrm{mL}$, respectively). 


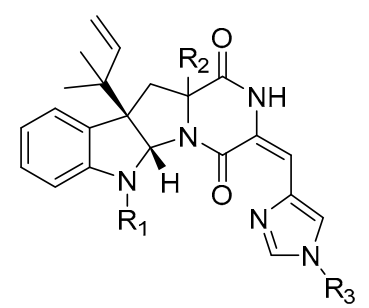

33: $\mathrm{R}_{1}=\mathrm{R}_{2}=\mathrm{R}_{3}=\mathrm{H}$

34: $\mathrm{R}_{1}=\mathrm{OCH}_{3}, \mathrm{R}_{2}=\mathrm{R}_{3}=\mathrm{H}$

35: $\mathrm{R}_{1}=\mathrm{H}, \mathrm{R}_{2}=\mathrm{OCH}_{3}, \mathrm{R}_{3}=$,

36: $\mathrm{R}_{1}=\mathrm{H}, \mathrm{R}_{2}=\mathrm{OCH}_{3}, \mathrm{R}_{3}=$, 年

37: $\mathrm{R}_{1}=\mathrm{H}, \mathrm{R}_{2}=\mathrm{OCH}_{3}, \mathrm{R}_{3}=\mathrm{H}$

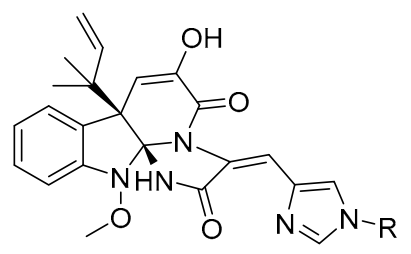

38: $\mathrm{R}=\mathrm{H}$

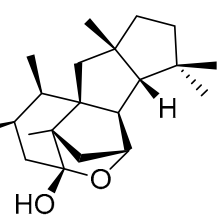<smiles>CCC(=O)CC(C)(C)C=[Ru]</smiles><smiles>[Z14]C=[R]C(C)(C)CC(=O)C=C(C)C</smiles>

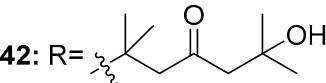

Figure 5. Chemical structures of compounds 33-42.
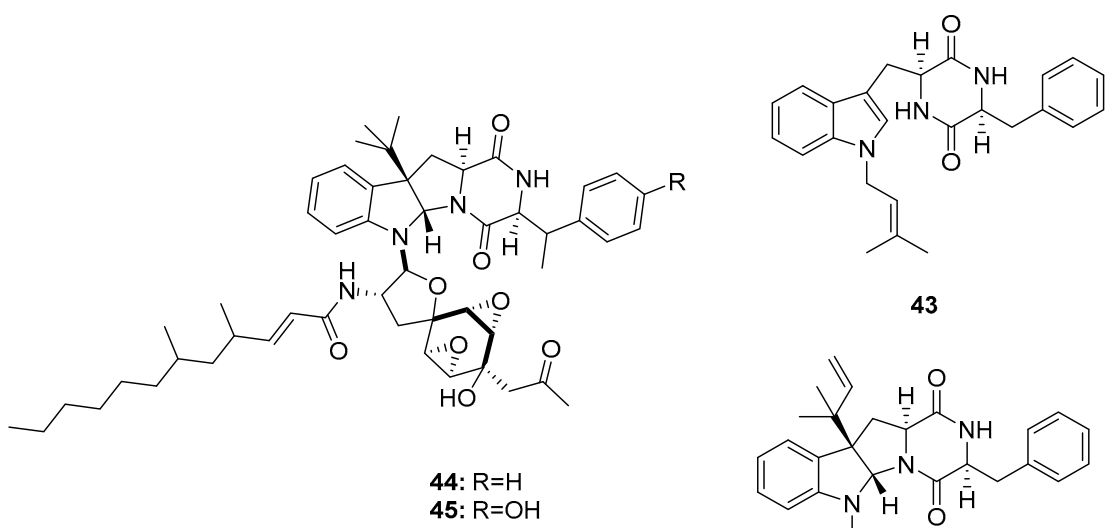

43

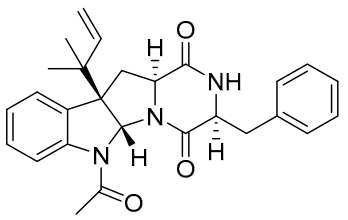

46

Figure 6. Chemical structures of compounds $\mathbf{4 4 - 4 6 .}$

Since the isolation of (+)-chaetocin A (47) and (+)-verticillin A (48) (Figure 7) in 1970, dimeric epidithiodiketopiperazine alkaloids have received much attention owing to their diverse biological activities and complex molecular structures [26,27]. In 1999, two additional dimeric epidithiodiketopiperazine alkaloids, (+)-11,11'-dideoxyverticillin A (49) and (+)-11'-deoxyverticillin A (50), were isolated from a marine alga-derived fungus Penicillium sp. and were found to exhibit extraordinary cytotoxicity against HCT-116 cells (human colon cancer) with $\mathrm{IC}_{50}$ of $30 \mathrm{ng} / \mathrm{mL}$ [28]. Chaetocin A (47) was the first compound reported to inhibit HMT, and to have specific effects on HMT SU(VAR)3-9 in vitro and in vivo [29]. (+)-11,11'-Dideoxyverticillin A (49), an alkaloid, exhibited diverse antitumor activities in vitro and in vivo [30]; in addition, it potently inhibited the phosphorylation of epidermal growth factor receptor in human breast cancer (MDA-MB-468) [31]. Movassaghi et al. used a concise enantioselective method for the total synthesis of (+)-11,11'-dideoxyverticillin A (49) in 2009 [32] based on mimicking the biosynthetic pathway; in addition, they used this approach to synthesize various dimeric epidithiodiketopiperazines [33]. 


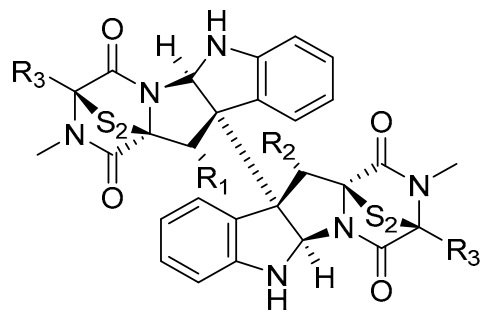

$$
\begin{aligned}
& \text { 47: } \mathrm{R}_{1}=\mathrm{R}_{2}=\mathrm{H}, \mathrm{R}_{3}=\mathrm{CH}_{2} \mathrm{OH} \\
& \text { 48: } \mathrm{R}_{1}=\mathrm{R}_{2}=\mathrm{OH}, \mathrm{R}_{3}=\mathrm{CH}_{3} \\
& \text { 49: } \mathrm{R}_{1}=\mathrm{R}_{2}=\mathrm{H}, \mathrm{R}_{3}=\mathrm{CH}_{3} \\
& \text { 50: } \mathrm{R}_{1}=\mathrm{H}, \mathrm{R}_{2}=\mathrm{OH}, \mathrm{R}_{3}=\mathrm{CH}_{3}
\end{aligned}
$$

Figure 7. Chemical structures of compounds $47-50$.

Seven cytotoxic indole diterpene alkaloids, penitrems A,B (51-52), D-F (53-55), paspaline (58), and emindole SB (59) (Figure 8) were isolated from a marine Penicillium sp. KBr-induced mutation of this fungus produced two bromo-substituted indole alkaloids, 6-bromopenitrems $\mathrm{B}$ and $\mathrm{E}$ (56-57) [34]. Compounds (51-59) showed potent antiproliferative $\left(\mathrm{IC}_{50}=5-20 \mu \mathrm{M}\right.$ for MCF-7; 8-30 $\mu \mathrm{M}$ for MDA-MB-231), anti-migratory ( $\mathrm{IC}_{50}=7-35 \mu \mathrm{M}$ for MDA-MB-231) and anti-invasive properties (IR\% $=10-75 \%$ at $15 \mu \mathrm{M}$ ) against human breast cancer cells. In addition, penitrems $\mathrm{A}$, $\mathrm{B}$, and $\mathrm{E}(\mathbf{5 1}-\mathbf{5 2}, \mathbf{5 4})$ were evaluated in 60 human tumor cell lines as a part of the Development Therapeutics Program of the National Cancer Institute (NCI60). Penitrem B (52) exhibited the strongest mean growth inhibitory effect in the 60 human cancer cells (IR $\%=41.05 \%$ at $10 \mu \mathrm{M})$ and was considered a potential selective inhibitory agent for leukemia cells. The nematode Caenorhabditis elegans was used to assess the brain's Maxi-K (BK) channel inhibitory activity and toxicity in vivo [35,36]. Penitrem A (51) and 6-bromopenitrem E (57) displayed BK channel inhibition, comparable to that of a knockout strain. A pharmacophore study on the effects of the penitrem skeleton on the antiproliferative activity against MCF-7 cells indicated that less structural complexity of the penitrems, paspaline (58), and emindole SB (59) better maintained the molecular alignment and pharmacophoric features. Penitrem A (51) was also considered a neurotoxin that antagonizes BK channels [37].
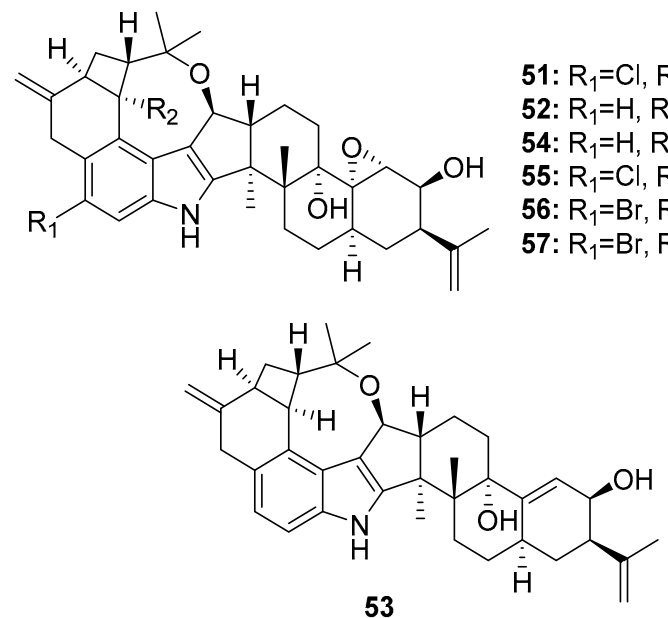

51: $\mathrm{R}_{1}=\mathrm{Cl}, \mathrm{R}_{2}=\mathrm{OH}$

52: $\mathrm{R}_{1}=\mathrm{H}, \mathrm{R}_{2}=\mathrm{H}$

54: $\mathrm{R}_{1}=\mathrm{H}, \mathrm{R}_{2}=\mathrm{OH}$

55: $\mathrm{R}_{1}=\mathrm{Cl}, \mathrm{R}_{2}=\mathrm{H}$

56: $\mathrm{R}_{1}=\mathrm{Br}, \mathrm{R}_{2}=\mathrm{H}$

57: $\mathrm{R}_{1}=\mathrm{Br}, \mathrm{R}_{2}=\mathrm{OH}$

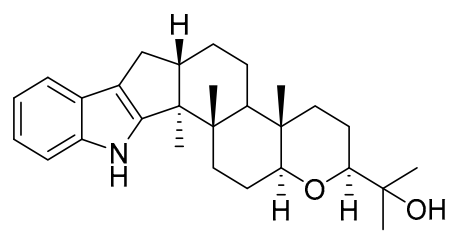

58

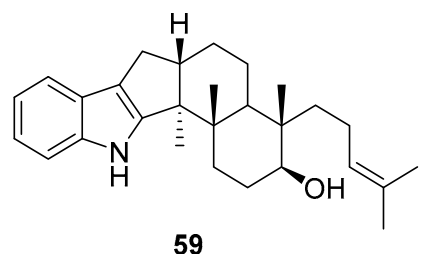

59

Figure 8. Chemical structures of compounds 51-59.

Another large family of indole alkaloid mycotoxins, comprising communesins A-D (60-63) (Figure 9), was isolated from marine-derived Penicillium sp. from a marine alga [38], marine sponge [39], and marine sediment [40]. Communesin B (61) (also called nomofungin) was more cytotoxic to P388 lymphocytic leukemia cells $\left(\mathrm{ED}_{50}=0.45 \mu \mathrm{g} / \mathrm{mL}\right)$ than communesin $\mathrm{A}(\mathbf{6 0})\left(\mathrm{ED}_{50}=3.5 \mu \mathrm{g} / \mathrm{mL}\right)$. 
The antiproliferative activity of communesins B-D (61-63) was further evaluated in six lymphocytic leukemia cell lines (U-937, THP-1, NAMALWA, L-428, MOLT-3, and SUP-B15). They steadily and effectively inhibited the proliferation of five of these cell lines with $\mathrm{ED}_{50}$ values ranging from 7 to $16 \mu \mathrm{g} / \mathrm{mL}$; however, they were inactive in L-428 cells. The total synthesis of communesin B (61) was previously reported [41].

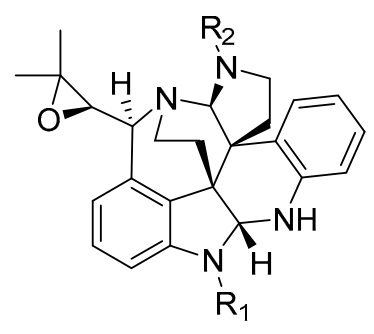

60: $\mathrm{R}_{1}=\mathrm{CH}_{3}, \mathrm{R}_{2}=\mathrm{COCH}_{3}$

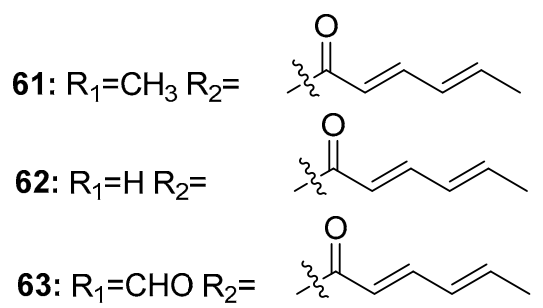

Figure 9. Chemical structures of compounds 60-63.

Four new cytotoxic prenylated indole alkaloid derivatives, penioxamide (64) [42], 13-O-prenyl-26-hydroxyverruculogen (65) [43], and penipalines B and C (66-67) (Figure 10) [44], were isolated from marine mangrove-derived P. oxalicum EN-201, marine sediment-derived P. brefeldianum SD-273, and marine sediment-derived P. paneum SD-44, respectively. Metabolites 64-65 showed significant lethality in brine shrimps with $\mathrm{LD}_{50}$ values of 5.6 and $9.4 \mu \mathrm{M}$, respectively (colchicine was employed as a positive control with an $\mathrm{LD}_{50}$ value of $7.8 \mu \mathrm{M}$ ). Metabolites 66-67 induced moderate cytotoxicity against $\mathrm{A} 549\left(\mathrm{IC}_{50}=20.44\right.$ and $21.54 \mu \mathrm{M}$, respectively) and HCT-116 cell lines $\left(\mathrm{IC}_{50}=14.88\right.$ and $18.54 \mu \mathrm{M}$, respectively).

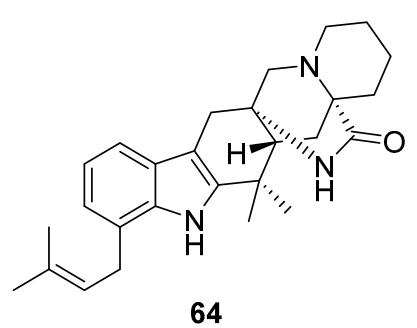

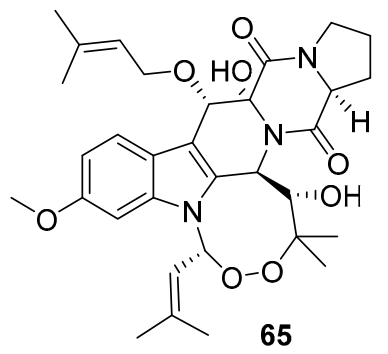<smiles>CC(C)=CCc1cccc2c3c([nH]c12)C(C)(C)NC(C(=O)O)C3</smiles>

66<smiles>C/C(=C/Cc1cccc2c(C=O)c[nH]c12)CO</smiles>

67

Figure 10. Chemical structures of compounds 64-67.

In addition, three 1,4-diazepane derivatives, terretriones A, C, and D (68-70) (Figure 11), obtained from marine sponge-derived P. vinaceum [45] and marine tunicate-derived Penicillium sp. CYE-87 [46], moderately inhibited the migratory activity of MDA-MB-231 cells with $\mathrm{IC}_{50}$ values of 17.7, 17.6, and $16.5 \mu \mathrm{M}$, respectively (Z-4-ethylthio-phenylmethylene hydantoin was used as a positive control with an $\mathrm{IC}_{50}$ value of $43.4 \mu \mathrm{M}$ ). These findings indicate that terretriones might be potential anti-metastatic breast cancer candidates. 
<smiles>[R]C([R])C1C(=O)C(Cc2ccccc2)C(=O)N(C)C1=O</smiles>

68: $\mathrm{R}_{1}=\mathrm{CH}_{3}, \mathrm{R}_{2}=\mathrm{H}, \mathrm{R}_{3}=(\mathrm{CH}) \mathrm{C}_{2} \mathrm{H}_{6}$

69: $\mathrm{R}_{1}=\mathrm{CH}_{3}, \mathrm{R}_{2}=\mathrm{CH}_{2} \mathrm{CH}_{3}, \mathrm{R}_{3}=\mathrm{CH}_{3}$

70: $\mathrm{R}_{1}=\mathrm{H}, \mathrm{R}_{2}=\mathrm{R}_{3}=\mathrm{CH}_{3}$

Figure 11. Chemical structures of compounds 68-70.

Six tetramic acid derivatives, penicillenols A1, A2, B1, B2, D1, and D2 (71-76) (Figure 12), were isolated from a marine sediment-derived fungus $P$. citrinum. Penicillenol B2 (74) exhibited the strongest cytotoxic activity against A-375 human malignant melanoma cell line $\left(\mathrm{IC}_{50}=0.97 \mu \mathrm{g} / \mathrm{mL}\right)$, whereas the $\mathrm{IC}_{50}$ values of compounds $71-73$ were $3.2,13.8$, and $2.8 \mu \mathrm{g} / \mathrm{mL}$, respectively $[47,48]$. Penicillenols D1 and D2 (75-76) showed moderate cytotoxicity against A549 cells with $\mathrm{IC}_{50}$ values of 17.2 and $12.1 \mu \mathrm{g} / \mathrm{mL}$, respectively. However, penicillenols A1 and B1 $(\mathbf{7 1}, 73)$ showed significant cytotoxicity in HL-60 cells ( $\mathrm{IC}_{50}=0.76$ and $3.2 \mu \mathrm{M}$, respectively) [49]. A novel tetramic acid derivative, penicitrinine A (77), which contains a citrinin-like group, was isolated [50]. The combination of two cytotoxic fragments in this metabolite might contribute to its extensive antiproliferative activity in diverse tumor cell lines, particularly A-375 cells. Penicitrinine A (77) not only induced A-375 cell apoptosis by upregulating Bax and downregulating Bcl-2, but also inhibited A-375 cell metastatic activity by suppressing matrix metalloproteinase 9 (MMP-9) and promoting the expression of its specific inhibitor, tissue inhibitor of metalloproteinases-1 (TIMP-1). These findings suggest that penicitrinine $\mathrm{A}(77)$ is a potential lead compound.

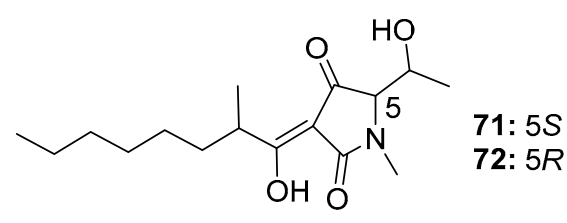

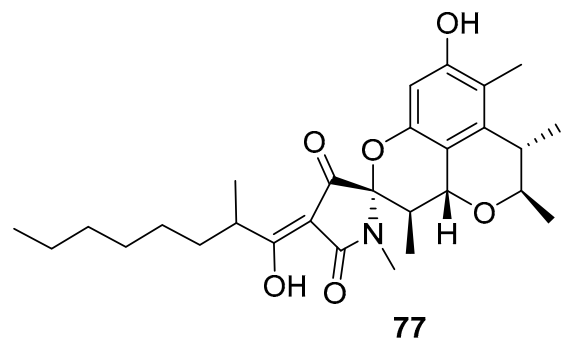<smiles>[R]C([R])=C1C(=O)C(=C(O)C(C)CCCCCC)C(=O)N1C</smiles>

73: $\mathrm{R}_{1}=\mathrm{CH}_{3}, \mathrm{R}_{2}=\mathrm{H} \Delta$ saturated 74: $\mathrm{R}_{1}=\mathrm{H}, \mathrm{R}_{2}=\mathrm{CH}_{3} \Delta$ saturated 75: $\mathrm{R}_{1}=\mathrm{CH}_{3}, \mathrm{R}_{2}=\mathrm{H} \Delta$ unsaturated 76: $\mathrm{R}_{1}=\mathrm{H}, \mathrm{R}_{2}=\mathrm{CH}_{3} \Delta$ unsaturated

Figure 12. Chemical structures of compounds 71-77.

Quinolinone and quinazolinone alkaloids have unique pharmacophores that allow their binding to multiple sites with high affinity; moreover, they possess various biological properties [51]. Some cytotoxic quinolinone (78-82) and quinazolinone alkaloids (83-85) (Figure 13) were isolated from marine-derived members of the Penicillium genus, such as P. janczewskii, Penicillium sp. ghq208, P. oxalicum 0312F1, P. chrysogenum EN-118, and P. commune SD-118 [23]. 2-quinolinone metabolites (78-79) exhibited IR\% values of $50-60 \%$ at $10 \mu \mathrm{g} / \mathrm{mL}$. Interestingly, compound 80 , which has an additional prenyl chain, showed significant cytotoxicity against MDA-MB-231 and HT-29 (human colon carcinoma) cell lines with IR\% values of $92-96 \%$ at $10 \mu \mathrm{g} / \mathrm{mL}$ [52]. In addition, a 4 -quinolinone derivative (82) exhibited significant cytotoxicity against the human lung cancer cell line 95-D 
$\left(\mathrm{IC}_{50}=0.57 \mu \mathrm{g} / \mathrm{mL}\right)$. Both compounds 81 and 82 exhibited similar cytotoxicity $\left(\mathrm{IC}_{50}=11.3\right.$ and 13.2 $\mu \mathrm{M}$, respectively) against HepG2 cells [53,54]. However quinazolinone derivatives (83-85) showed only moderate cytotoxicity (compound 83, $\mathrm{IC}_{50}=20 \mu \mathrm{g} / \mathrm{mL}$ in SW1990 cell line; compound 84, $\mathrm{IC}_{50}=8 \mu \mathrm{g} / \mathrm{mL}$ in DU145, A549, and Hela cell lines; and compound 85, IR\% $=35-40$ at $200 \mu \mathrm{g} / \mathrm{mL}$ in SGC-7901 and BEL-7404 cell lines) [55,56].<smiles>[R]C1([R])C(=O)Nc2ccccc2[C@]1(O)c1ccc(OC)cc1</smiles>

78: $\mathrm{R}_{1}=\mathrm{H}, \mathrm{R}_{2}=\mathrm{OH}$ 79: $\mathrm{R}_{1}=\mathrm{OH}, \mathrm{R}_{2}=\mathrm{H}$<smiles>[R]OC(=O)c1c(-c2ccc[nH]2)[nH]c2ccccc2c1=O</smiles>

81: $\mathrm{R}=\mathrm{CH}_{3}$ 82: $\mathrm{R}=\mathrm{H}$<smiles>COc1ccc([C@]2(O)c3c(ccc(CC=C(C)C)c3O)NC(=O)[C@H]2OC)cc1</smiles>

80

Figure 13. Chemical structures of compounds 78-85.

In an ongoing study that aims to produce new active metabolites from P. paneum SD-44 (a deep sea sediment-derived fungus) using culture variations, three amidine anthranilic acid analogues (86-88) and one triazole anthranilic acid derivative, penipanoid A (89) (Figure 14), were obtained after culture in malt and rice medium, respectively. Compounds 86 and 87 strongly inhibited RKO human colon cancer cell viability ( $\mathrm{IC}_{50}=8.4$ and $9.7 \mu \mathrm{M}$, respectively). In addition, compound 88 was cytotoxic to Hela cells $\left(\mathrm{IC}_{50}=6.6 \mu \mathrm{M}\right)$ [57], whereas compound 89 with a triazole group only weakly inhibited SMMC-7721 cell viability (human hepatocarcinoma) $\left(\mathrm{IC}_{50}=54.2 \mu \mathrm{M}\right)$ while fluorouracil was used as a positive control for three cell lines with $\mathrm{IC}_{50}$ values of 25.0, 14.5, and $13.0 \mu \mathrm{M}$, respectively [58].<smiles>C/C(=N\CC(C)(C)O)Nc1ccccc1C(=O)O</smiles>

86<smiles>O=C(O)c1ccccc1N/C=N/c1ccco1</smiles>

87<smiles>CC(=O)/C(C)=N/c1ccccc1C(=O)O</smiles>

88<smiles>O=C(O)c1ccccc1-n1ncnc1Cc1ccc(O)cc1</smiles>

Figure 14. Chemical structures of compounds 86-89.

An azaphilone analogue, bis-sclerotioramin (90) (Figure 15), obtained from a marine mangrove endophytic fungus, Penicillium 303\#, was found to possess moderate cytotoxicity against MDA-MB-435 
cell line ( $\mathrm{IC}_{50}=7.13 \mu \mathrm{g} / \mathrm{mL}$ ), while epirubicin was used as a positive control with an $\mathrm{IC}_{50}$ value of $0.325 \mu \mathrm{g} / \mathrm{mL}$ [59]. Another novel alkaloid, the sorbicilin-derived sorbicillactone A (91), was first isolated from a Mediterranean sponge-derived fungus, P. chrysogenum. Sorbicillactone A (91) exhibited a selective antileukemic activity in L5178Y cells (murine leukemic lymphoblast) with an $\mathrm{IC}_{50}$ of $2.2 \mu \mathrm{g} / \mathrm{mL}$, as well as in other tumor cell lines ( $\mathrm{IC}_{50}>10 \mu \mathrm{g} / \mathrm{mL}$ ). The biosynthesis of sorbicillactone A (91) was investigated using ${ }^{13} \mathrm{C}$-labeled precursor feeding experiments, which showed that the its skeleton was derived from acetate, alanine, and methionine [60]. Furthermore, a new strategy for the large-scale biotechnological production of sorbicilin-derived alkaloids was developed for preclinical screening and a structure-activity relationship (SAR) study [61]. In addition, a 4-oxoquinoline derivative, brocaeloid B (92), isolated from the mangrove endophytic fungus $P$. brocae, showed mild lethality against brine shrimps with an $\mathrm{LD}_{50}$ of $36.7 \mu \mathrm{M}$, while colchicine was used as a positive control with an $\mathrm{LD}_{50}$ value of $87.6 \mu \mathrm{M}$ [62]. Li et al. cultured the marine mangrove fungus $P$. varibile with the DNA methyltransferase inhibitor 5-azacytidine to identify novel responsive molecules by gene silencing. A highly modified fatty acid amide, varitatin A (93), exhibited significant cytotoxicity against HCT-116 cells $\left(\mathrm{IC}_{50}=2.8 \mu \mathrm{M}\right.$, while doxorubicin was used as a positive control with an $\mathrm{IC}_{50}$ value of $0.2 \mu \mathrm{M}$ ) and potently inhibited protein tyrosine kinases, platelet-derived growth factor receptor-beta (PDGFR- $\beta$ ), and ErbB4 with IR\% values of 50 and $40 \%$, respectively, at a concentration of $1 \mu \mathrm{M}$ [63]. In addition, a new pyridinyl- $\alpha$-pyrone alkaloid, 18-hydroxydecaturin B (94), was isolated from an endophytic fungus, P. oxalicum EN-201, derived from the marine mangrove Rhizophora stylosa. Compound 94 showed significant lethality in brine shrimps $\left(\mathrm{LD}_{50}=2.3 \mu \mathrm{M}\right.$, while colchicine was used as a positive control with an $\mathrm{LD}_{50}$ value of $7.8 \mu \mathrm{M}$ ) [42]. A previous study showed that the metabolites of decaturin, a potent insecticide, were cytotoxic [64]. The isocyanide alkaloid, xantocillin X (95), which is a known antiviral and antibiotic agent [65], was first isolated from P. notatum in 1950 [66]. Recently, compound 95 was isolated from the deep sea sediment-derived fungus P. commune SD-118, and showed moderate cytotoxicity in six cancer cell lines (MCF-7, HepG2, NCI-H460, Hela, DU145, and MDA-MB-231) with $\mathrm{IC}_{50}$ values of $12,7,10,10,8$, and $8 \mu \mathrm{g} / \mathrm{mL}$, respectively, while fluorouracil was used as a positive control with $\mathrm{IC}_{50}$ values of $4,14,1,14,0.4$, and $8 \mu \mathrm{g} / \mathrm{mL}$, respectively [23]. A later pharmacological study on human HepG2 cells showed that compound 95 induced apoptosis and autophagy by inhibiting the MEK/EPK signaling pathway and activating the class III PI3K/Beclin 1 signaling pathway [67].

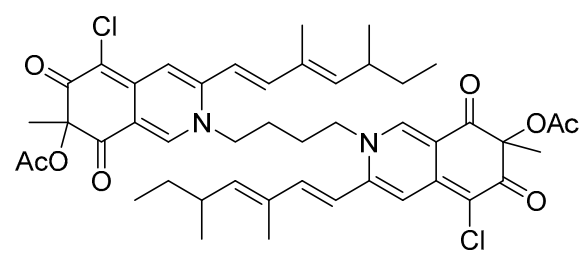<smiles>C/C=C/C=C/C(=O)NC1(C)C(=O)OC2(C)C(O)=C(C)C(=O)C(O)=C12</smiles>

91<smiles>C=CC(C)(C)C(=O)Nc1ccccc1C(=O)CCNC(C)=O</smiles>

92

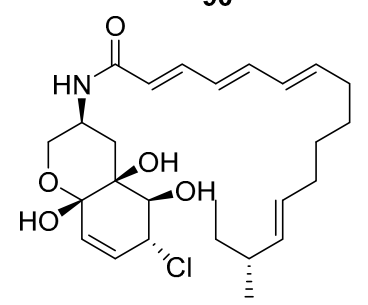

93

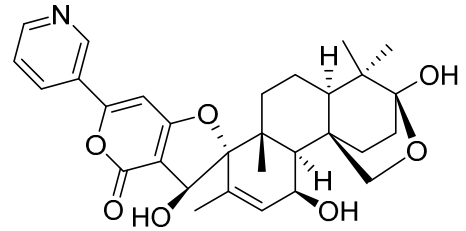

94<smiles>C=[N+]C(=C\c1ccc(O)cc1)/C(=C\c1ccc(O)cc1)[N+](=C)C</smiles>

95

Figure 15. Chemical structures of compounds 90-95. 


\section{Terpenes, Meroterpenes, and Steroids}

The genus Penicillium is a well-known producer of eremophilane-type sesquiterpenes with phytotoxic, mycotoxic, and phytohormonic activities [68,69]. Chemical investigation of an Antarctic deep sea-derived fungus, Penicillium sp. PR19 N-1, yielded three new cytotoxic eremophilane-type sesquiterpenes (96-98) (Figure 16), which were moderately cytotoxic to HL-60 $\left(\mathrm{IC}_{50}=45.8,28.3\right.$, and $11.8 \mu \mathrm{M}$, respectively) and $\mathrm{A} 549\left(\mathrm{IC}_{50}=82.8,5.2\right.$, and $12.2 \mu \mathrm{M}$, respectively) cancer cell lines [70,71]. Three other eremophilane-type sesquiterpenes (99-101) were isolated from a sea mud-derived fungus, Penicillium sp. BL 27-2. Of these, compound 99 was the most cytotoxic to P388, A549, HL-60, and BEL-7402 cell lines $\left(\mathrm{IC}_{50}=0.073,0.096,0.065\right.$, and $4.59 \mu \mathrm{M}$, respectively), whereas compounds $\mathbf{1 0 0}$ and $\mathbf{1 0 1}$ had $\mathrm{IC}_{50}$ values in the range of 3-12 $\mu \mathrm{M}$ [72]. These results suggest that the epoxide ring is essential for the cytotoxicity of eremophilane-type sesquiterpenes and that the presence of an acetyl group enhances the cytotoxicity. A new acorane sesquiterpene, adametacorenol B (102), isolated from a marine sponge-derived fungus, P. adametzioides AS-53, displayed selective cytotoxicity against NCI-H446 cell lines $\left(\mathrm{IC}_{50}=5 \mu \mathrm{M}\right)$, compared to its cytotoxicity against the other 13 tumor cell lines tested (A549, DU145, HeLa, HepG2, Huh-7 (human hepatocarcinoma), L02 (human hepatocarcinoma), LM3 (murine breast cancer), MA (mouse Leydig tumor), MCF-7, SGC-7901, SW1990, SW480, and U251) $\left(\mathrm{IC}_{50}>10 \mu \mathrm{M}\right)[17]$.<smiles>C=C(C)C1CC2(C)C(=CC(O)CC2C)CC1O</smiles>

96<smiles>CC(=O)OC1C=C(Cl)C2=CC(=O)C(O)=CC2(C)C1[18O]</smiles>

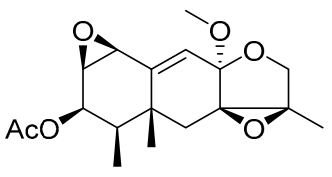

97

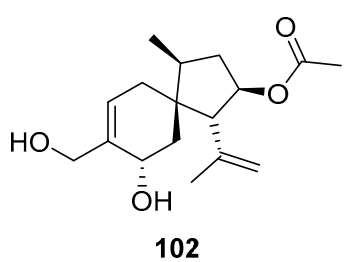<smiles>[R]C1C=C2CCC([R1])C(C)C2(C)C(=O)C1=CC(=C)C</smiles>

99: $R_{1}=O A c, R_{2}==0$ 100: $\mathrm{R}_{1}=\mathrm{OH}, \mathrm{R}_{2}==\mathrm{O}$ 101: $\mathrm{R}_{1}=\mathrm{OH}, \mathrm{R}_{2}=\beta \mathrm{OH}$

Figure 16. Chemical structures of compounds 96-102.

The deep sea sediment-derived fungus Penicillium sp. was reported to be a good source of cytotoxic diterpenes. Six tetracyclic diterpenes, conidiogenones B-G (103-108) (Figure 17), exhibited cytotoxicity against HL-60, A549, BEL-7402, and MOLT-4 cell lines. Conidiogenone C (104) was potently cytotoxic against HL-60 and BEL-7402 cells with $\mathrm{IC}_{50}$ values of 0.038 and $0.97 \mu \mathrm{M}$, respectively; however, it was not cytotoxic against A549 and MOLT-4 cell lines at $50 \mu \mathrm{M}$. Other conidiogenones (103, 105-108) had moderate cytotoxicity with $\mathrm{IC}_{50}$ values ranging from 1 to $50 \mu \mathrm{M}$ [22]. The spiroditerpenes, breviones I and A (109-110) were also obtained from this fungus and showed cytotoxicity comparable to that of cisplatin (the positive control) against MCF-7 cells $\left(\mathrm{IC}_{50}=7.44\right.$ and $28.4 \mu \mathrm{M}$, respectively, versus $8.04 \mu \mathrm{M}$ for cisplatin) [73].

Although several marine-derived steroids have been isolated, few have been found to be bioactive. A cytotoxic polyoxygenated steroid, penicisteroide A (111) (Figure 18), was isolated from a marine alga-derived fungus, $P$. chrysogenum QEN-24S. Penicisteroide A (111) displayed moderate cytotoxicity against Hela, SW1990, and NCI-H460 cell lines with $\mathrm{IC}_{50}$ values of 15,31 , and $40 \mu \mathrm{g} / \mathrm{mL}$, respectively [74]. Three other polyoxygenated steroids (112-114) and two epidioxygenated steroids (115-116) were isolated from the marine moss-derived fungus Penicillium sp. These steroids moderately inhibited HepG2 cell line growth ( $\mathrm{IC}_{50}$ values $=10.4,15.6,20.7,16.8$, and $21.3 \mu \mathrm{g} / \mathrm{mL}$, respectively) [75]. In addition, an epidioxygenated steroid (117), produced by a sea squirt-derived fungus, $P$. stoloniferum QY2-10, was cytotoxic to P388 cells with an $\mathrm{IC}_{50}$ of $4.07 \mu \mathrm{M}$ [76]. Moreover, a marine Penicillium sp. fungus collected from the inner tissues of an unidentified sponge is reportedly the source 
of two epimeric steroids (118-119) and two cytotoxic steroids of a new class, dankasterone A (120) and B (121). Dankasterone A (120) was more effective than the positive control, adriamycin $\left(\mathrm{IC}_{50}=0.98 \mu \mathrm{M}\right)$ against HL-60, Hela, and $\mathrm{K} 562$ cancer cell lines with $\mathrm{IC}_{50}$ values of $0.78,4.11$, and $7.57 \mu \mathrm{M}$, respectively. Compounds 118-119 and $\mathbf{1 2 1}$ also significantly inhibited K562 cell growth $\left(\mathrm{IC}_{50}=4.38,5.54\right.$, and $7.89 \mu \mathrm{M}$, respectively) [77].

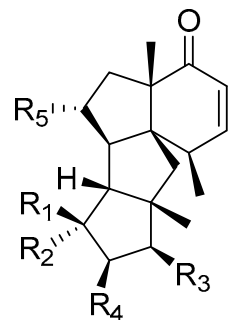

103: $\mathrm{R}_{1}=\mathrm{R}_{2}=\mathrm{CH}_{3}, \mathrm{R}_{3}=\mathrm{R}_{4}=\mathrm{R}_{5}=\mathrm{H}$

104: $\mathrm{R}_{1}=\mathrm{CH}_{2} \mathrm{OH}, \mathrm{R}_{2}=\mathrm{CH}_{3}, \mathrm{R}_{3}=\mathrm{R}_{4}=\mathrm{R}_{5}=\mathrm{H}$

105: $\mathrm{R}_{1}=\mathrm{CH}_{3}, \mathrm{R}_{2}=\mathrm{CH}_{2} \mathrm{OH}, \mathrm{R}_{3}=\mathrm{R}_{4}=\mathrm{R}_{5}=\mathrm{H}$

106: $\mathrm{R}_{1}=\mathrm{R}_{2}=\mathrm{CH}_{3}, \mathrm{R}_{3}=\mathrm{OH}, \mathrm{R}_{4}=\mathrm{R}_{5}=\mathrm{H}$

107: $\mathrm{R}_{1}=\mathrm{R}_{2}=\mathrm{CH}_{3}, \mathrm{R}_{3}=\mathrm{H}, \mathrm{R}_{4}=\mathrm{OH}, \mathrm{R}_{5}=\mathrm{H}$

108: $\mathrm{R}_{1}=\mathrm{R}_{2}=\mathrm{CH}_{3}, \mathrm{R}_{3}=\mathrm{H}, \mathrm{R}_{4}=\mathrm{H}, \mathrm{R}_{5}=\mathrm{OH}$

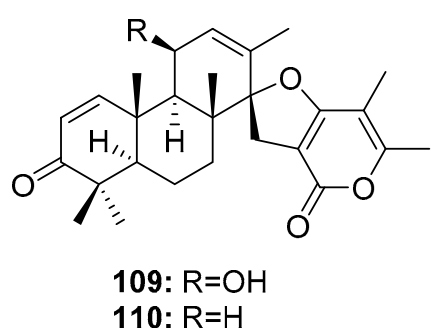

110: $\mathrm{R}=\mathrm{H}$

Figure 17. Chemical structures of compounds 103-110.

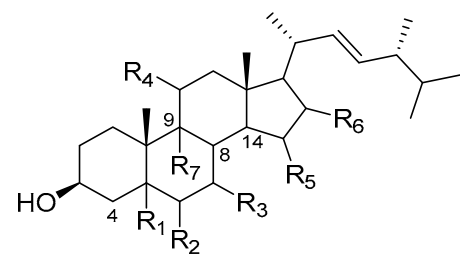

111: $\mathrm{R}_{1}=\mathrm{R}_{5}=\mathrm{R}_{7}=\mathrm{H}, \mathrm{R}_{2}=\mathrm{R}_{3}=\mathrm{R}_{4}=\beta \mathrm{OH}, \mathrm{R}_{6}=\beta \mathrm{OAc}$

112: $R_{1}=R_{3}=\alpha O H, R_{2}=\beta O H, R_{4}=R_{5}=R_{6}=R_{7}=H, \Delta_{8-14}$ unsaturated

113: $R_{1}=R_{3}=\alpha O H, R_{2}=\beta O H, R_{4}=R_{5}=R_{6}=R_{7}=H, \Delta_{8-9}$ unsaturated

114: $\mathrm{R}_{1}=\mathrm{R}_{7}=\alpha \mathrm{OH}, \mathrm{R}_{2}==\mathrm{O}, \mathrm{R}_{3}=\mathrm{R}_{4}=\mathrm{R}_{5}=\mathrm{R}_{6}=\mathrm{H}, \Delta_{7-8}$ unsaturated

118: $R_{2}=R_{4}=R_{6}=R_{7}=H, R_{3}==O, R_{5}=\beta O H, \Delta_{4-5} \Delta_{8-14}$ unsaturated

119: $R_{2}=R_{4}=R_{6}=R_{7}=H, R_{3}==O, R_{5}=\alpha O H, \Delta_{4-5} \Delta_{8-14}$ unsaturated
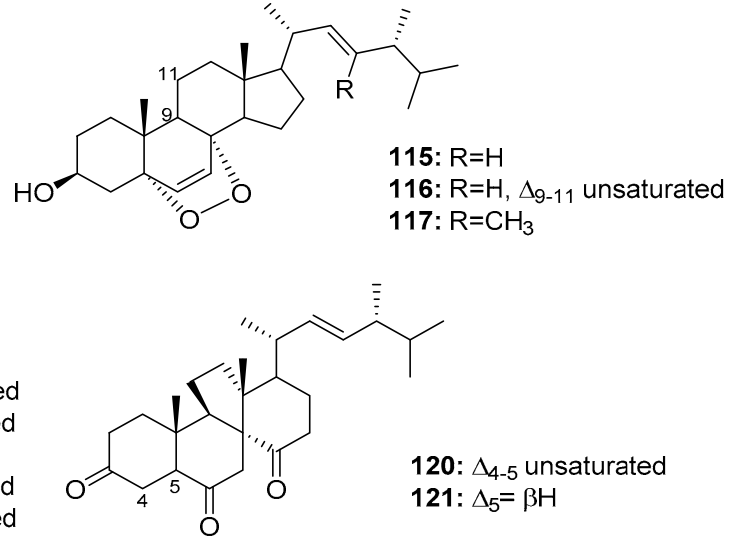

Figure 18. Chemical structures of compounds 111-121.

Meroterpenes are widely distributed in the marine environment, particularly in brown algae and microorganisms. Terpene-quinone and -hydroquinone are the major bioactive members because they produce reactive oxygen species (ROS) [78]. Three quinone- and hydroquinone-type meroterpenes (122-124) (Figure 19) were isolated from a marine-derived Penicillium sp. Compounds 122 and 123 exhibited extensive cytotoxicity against five cancer cell lines (A549, SKOV-3 (human ovary adenocarcinoma), SKMEL-2 (human skin cancer), XF498 (human CNS cancer), and HCT15 (human colon cancer)) with $\mathrm{IC}_{50}$ values in the range of $3-10 \mu \mathrm{g} / \mathrm{mL}$, whereas compound $\mathbf{1 2 4}$ had $\mathrm{IC}_{50}$ values ranging from 20 to $40 \mu \mathrm{g} / \mathrm{mL}$ (doxorubicin was used as a positive control with $\mathrm{IC}_{50}$ values of $0.02 \sim 0.8 \mu \mathrm{g} / \mathrm{mL}$ ). These results suggest that the quinone form tends to be less cytotoxic [79]. Penicillone A (125), isolated from marine-derived Penicillium sp. F11., contains a carboxylic acid group instead of the isoprenyl tail, which resulted in mild cytotoxicity against fibrosarcoma (HT1080) and human nasopharyngeal carcinoma (Cne2) cell lines ( $\mathrm{IC}_{50}=45.8$ and $46.2 \mu \mathrm{M}$, respectively) [80].

Two sesquiterpene $\alpha$-pyrones, phenylpyropenes $E$ and $F$ (126-127) (Figure 20), were isolated from the marine-derived fungus $P$. concentricum ZLQ-69 and displayed moderate and selective cytotoxicity against MGC-803 cells (human gastric cancer) with $\mathrm{IC}_{50}$ values of 19.1 and $13.6 \mu \mathrm{M}$, respectively (doxorubicin was used as a positive control with an $\mathrm{IC}_{50}$ value of $0.37 \mu \mathrm{M}$ ) [81]. Furthermore, the marine sediment-derived fungus Penicillium sp. F446 yielded two new sesquiterpene $\gamma$-pyrone-type meroterpenes, penicillipyrone A and B (128-129), which were moderately cytotoxic against A549 cells $\left(\mathrm{IC}_{50}=15\right.$ and $17 \mu \mathrm{M}$, respectively, while doxorubicin was used as a positive control with 
an $\mathrm{IC}_{50}$ value of $\left.1.2 \mu \mathrm{M}\right)$ [82]. Two polycyclic $\alpha$-pyrone-type meroterpenes (130-131), isolated from the marine mangrove endophytic fungus Penicillium 303\#, exhibited $\mathrm{IC}_{50}$ values of $20-30 \mu \mathrm{g} / \mathrm{mL}$ in four cancer cell lines (MDA-MB-435, HepG2, HCT-116, and A549), while epirubicin was used as a positive control with $\mathrm{IC}_{50}$ values of $0.2 \sim 0.6 \mu \mathrm{g} / \mathrm{mL}$ [59]. Fumagillin was first isolated from Aspergillus fumigatus in 1949, and has been used as an antimicrobial [83]. Recently, ligerin (132), a natural chlorinated merosesquiterpene related to fumagillin, was obtained from a marine-derived Penicillium sp., and showed selective in vitro antiproliferative activity against osteosarcoma cell lines $\left(\mathrm{IC}_{50}=117 \mathrm{nM}\right.$ against POS1 cells, which is 20 times greater than the $\mathrm{IC}_{50}$ in other cancer cell lines), while doxorubicin was used as a positive control with $\mathrm{IC}_{50}$ values of $0.04 \sim 2 \mu \mathrm{M}$ [84]. Ligerin analogues were semi-synthesized in an SAR study, which showed that chlorohydrin and C6 substituents were crucial for cytotoxic activities. Furthermore, ligerin (132) exhibited stronger cytotoxicity against human osteosarcoma SaOS2 and MG63 cancer cell lines. However, its cytotoxicity was less than that of TNP470 (a positive control and fumagillin analogue) [85].<smiles>CC(C)=CCC/C(C)=C/CC/C(C)=C/CC12OC1C(O)C(C)=CC2=O</smiles>

122<smiles>CC(C)=CCC/C(C)=C/CC/C(C)=C/CC1=CC(=O)C(C)=CC1=O</smiles><smiles>CC(C)=CCC/C(C)=C/CC/C(C)=C/Cc1cc(O)c(C)cc1O</smiles><smiles>CC1=CC(=O)C(O)(C/C=C(\C)CC/C=C(\C)CCC(=O)O)[C@H](O)[C@@H]1O</smiles>

Figure 19. Chemical structures of compounds 122-125.

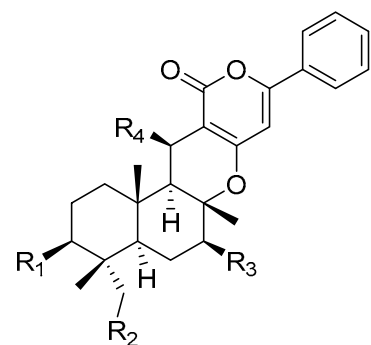

126: $\mathrm{R}_{1}=\mathrm{R}_{4}=\mathrm{OH}, \mathrm{R}_{2}=\mathrm{R}_{3}=\mathrm{OCOCH}_{3}$ 127: $\mathrm{R}_{1}=\mathrm{OCOCH}_{3}, \mathrm{R}_{2}=\mathrm{R}_{3}=\mathrm{R}_{4}=\mathrm{H}$

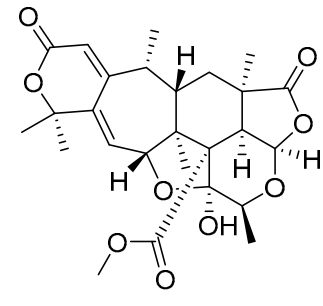

130

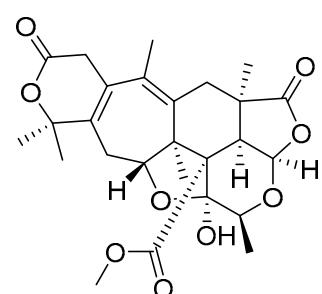

131<smiles>[R]C1CC[C@@]2(C)[C@H](CC[C@H]1C)[C@@H]1Cc3c(oc(C)cc3=O)C[C@H]12</smiles>

128: $\mathrm{R}=\beta \mathrm{OH}$ 129: $R==O$

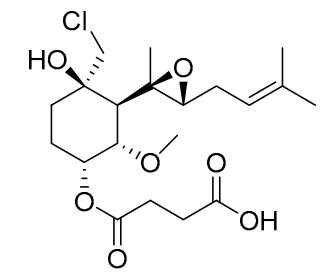

132

Figure 20. Chemical structures of compounds 126-132. 


\section{Polyketides}

Chromone derivatives are abundantly present in nature and are considered potential immunomodulatory, anticancer, and anti-inflammatory agents. Chromone scaffolds were reported to possess outstanding pharmacological properties [86]. A Chinese research group recently isolated four dihydrothiophene-condensed chromones, oxalicumones D, E (133-134) and A, B (135-136) (Figure 21) from a marine gorgonian-derived fungus, P. oxalicum SCSGAF 0023. Similar to synthetic dihydrothiophene-condensed chromones (137-144), these four natural chromones (133-136) displayed significant cytotoxicity against eight carcinoma cell lines (human lung adenocarcinoma (H1975), human lymphoma (U937), K562, BGC823, MOLT-4. MCF-7, HL-60, and Huh-7) ( $\left.\mathrm{IC}_{50}<10 \mu \mathrm{M}\right)$. Of these, oxalicumone A (135) was the most cytotoxic against MOLT-4 cell line $\left(\mathrm{IC}_{50}=0.30 \mu \mathrm{M}\right)$. An SAR study showed that the 2,3-dihydrothiophene unit was crucial for activity and that the presence of 1-OH and absolute configuration at C-6 contributed to cytotoxicity $[87,88]$. Subsequent pharmacological studies showed that oxalicumone A (135) inhibited leukemia cell growth and induced apoptosis, in part, via the induction of the endoplasmic reticulum stress pathway by upregulating calnexin and Bax and activating unfolded protein response [89]. Another study found that oxalicumone A (135) could induce oxidative stress injury in the mitochondria, and thus promote human renal epithelial cell death [90]. Chromosulfine (145), a novel cyclopentachromone sulfide which is structurally similar to dihydrothiophene-condensed chromones, was isolated from a neomycin-resistant mutant of the marine-derived fungus $P$. purpurogenum G59, and showed selective cytotoxicity against HL-60 cancer cell line $\left(\mathrm{IC}_{50}=16.7 \mu \mathrm{M}\right)$ [91]. Secalonic acid $\mathrm{F}(146)$, a chiral dimeric tetrahydroxanthone, was first isolated from Aspergillus sp. before discovering that the deep sea sediment-derived fungus Penicillium sp. F11 is a good source of this compound. Compound 146 induced HL-60 cell apoptosis by modulating the Rho GDP dissociation inhibitor 2 pathway [92]. Recent studies showed that secalonic acid $\mathrm{F}$ (146) could induce apoptosis by activating caspase 3 and 9 through the mitochondrial pathway in hepatocellular carcinoma, wherein it was found to be more effective than 5-fluorouracil [93]. Furthermore, a flavone, namely penimethavone A (147), obtained from a gorgonian-derived fungus, $P$. chrysogenum, exhibited selective cytotoxicity against Hela and rhabdomyosarcoma cell lines $\left(\mathrm{IC}_{50}=8.41\right.$ and $8.18 \mu \mathrm{M}$, respectively) while adriamycin was used as a positive control with $\mathrm{IC}_{50}$ values of 0.43 and $0.09 \mu \mathrm{M}$, respectively [94].

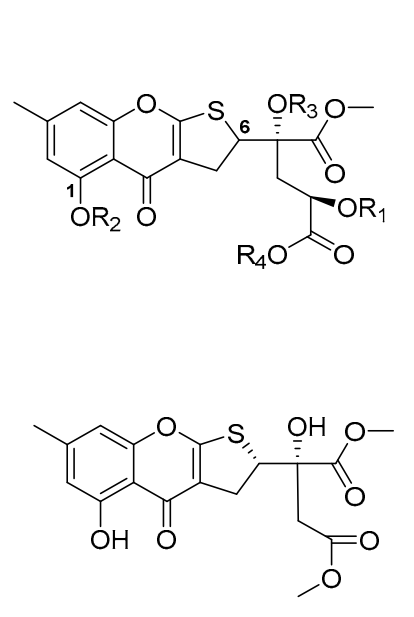

134
133: $6 S, \mathrm{R}_{1}=\mathrm{R}_{2}=\mathrm{R}_{3}=\mathrm{H}, \mathrm{R}_{4}=\mathrm{H}$ 135: $6 S, R_{1}=R_{2}=R_{3}=H, R_{4}=C_{3}$ 136: $6 R, \mathrm{R}_{1}=\mathrm{R}_{2}=\mathrm{R}_{3}=\mathrm{H}, \mathrm{R}_{4}=\mathrm{CH}_{3}$ 137: $6 S, \mathrm{R}_{1}=(R)-M T P A, \mathrm{R}_{2}=\mathrm{R}_{3}=\mathrm{H}, \mathrm{R}_{4}=\mathrm{CH}_{3}$ 138: $6 S, \mathrm{R}_{1}=(S)-M T P A, \mathrm{R}_{2}=\mathrm{R}_{3}=\mathrm{H}, \mathrm{R}_{4}=\mathrm{CH}_{3}$ 139: $6 S, \mathrm{R}_{1}=\mathrm{NC}, \mathrm{R}_{2}=\mathrm{R}_{3}=\mathrm{H}, \mathrm{R}_{4}=\mathrm{CH}_{3}$ 140: $6 S, R_{1}=R_{2}=N C, R_{3}=H, R_{4}=C_{3}$ 141: $6 S, \mathrm{R}_{1}=\mathrm{BTBC}, \mathrm{R}_{2}=\mathrm{R}_{3}=\mathrm{H}, \mathrm{R}_{4}=\mathrm{CH}_{3}$ 142: $6 S, \mathrm{R}_{1}=\mathrm{R}_{2}=\mathrm{R}_{3}=B T B C, \mathrm{R}_{4}=\mathrm{CH}_{3}$ 143: $6 S, \mathrm{R}_{1}=\mathrm{R}_{2}=\mathrm{BC}, \mathrm{R}_{3}=\mathrm{H}, \mathrm{R}_{4}=\mathrm{CH}_{3}$ 144: $6 R, \mathrm{R}_{1}=(S)-M T P A, \mathrm{R}_{2}=\mathrm{R}_{3}=\mathrm{H}, \mathrm{R}_{4}=\mathrm{CH}_{3}$<smiles>Cc1cc(O)cc(O)c1C1CC(=O)c2c(O)cc(O)cc2O1</smiles><smiles>COC(=O)[C@H](O)CS[C@H]1Cc2c(oc3cc(C)cc(O)c3c2=O)[C@]1(O)C(=O)OC</smiles>

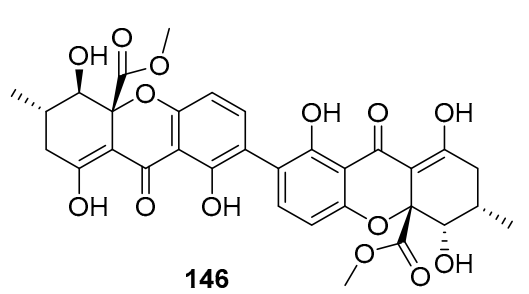

Figure 21. Chemical structures of compounds 133-147.

Coumarin derivatives of the chromone isomers (148-150) (Figure 22) were also isolated from the deep sea sediment-derived fungus (P. chrysogenum SCSIO 41001), a marine sponge-derived fungus Penicillium sp., and a mangrove endophytic fungus (Penicillium sp. ZH16), respectively. The dimeric 
isocoumarin, bipenicillisorin (148), displayed significant cytotoxicity against K562, A549, and Huh-7 cell lines $\left(\mathrm{IC}_{50}=6.78,6.94\right.$, and $2.59 \mu \mathrm{M}$, respectively), while taxol was used as a positive control with $\mathrm{IC}_{50}$ values of 3.44, 2.61, and $14.70 \mathrm{nM}$, respectively [95]. The dihydroisocoumarin monocerin (149) exhibited significant cytotoxicity against L5178Y cells (a murine lymphoma cell line) with an $\mathrm{IC}_{50}$ value of $8.4 \mu \mathrm{M}$ (kahalalide F was used as a positive control with an $\mathrm{IC}_{50}$ value of $4.3 \mu \mathrm{M}$ ) [96]. Moreover, furanocoumarin (150) showed moderate cytotoxicity against human nasopharyngeal carcinoma (KB and $\mathrm{KBv} 200$ ) cell lines ( $\mathrm{IC}_{50}=5$ and $10 \mu \mathrm{g} / \mathrm{mL}$, respectively) [97].<smiles>COC(=O)C1Cc2cc(OC)c(-c3c(OC)cc4c(c3O)C(=O)OC(C(=O)OC)C4)c(O)c2C(=O)O1</smiles><smiles>CCCC1CC2OC(=O)c3c(cc(OC)c(OC)c3O)C2O1</smiles><smiles>CC(C)=CCc1c2occc2c(C)c2ccc(=O)oc12</smiles>

Figure 22. Chemical structures of compounds 148-150.

Citrinin (151) (Figure 23), a typical azaphilone polyketide mycotoxin, was first found in P. citrinum in 1931 [98]. Compound $\mathbf{1 5 1}$ is strongly nephrotoxic because of its inhibition of respiration complex III [99]. The biosynthesis pathway of compound 151 was further investigated [100]. Interestingly, the marine sponge-derived fungus Penicillium sp. FF001 was found to be a good source of unique and potent citrinin derivatives [101]. Two new citrinin derivatives, penicitrinols $\mathrm{L}$ and M (152-153), isolated from the marine sediment-derived fungus P. citrinum, showed moderate cytotoxicity against a human Caucasian colon adenocarcinoma cell line (SW-620) $\left(\mathrm{IC}_{50}=25.6\right.$ and $20.9 \mu \mathrm{M}$, respectively) [48]. One penicitrinol analogue, berkelic acid (154), with a novel spiroketal structure, isolated from an acid mine lake fungal extremophile Penicillium sp., showed selective and extraordinary cytotoxicity against a human ovarian carcinoma cell line (OVCAR-3) at nanomolar concentrations $\left(\mathrm{GI}_{50}=91 \mathrm{~nm}\right)$ [102]. The total synthesis of $(-)$-berkelic acid (154) was previously described [103]. An alga-derived fungus, $P$. thomii, yielded a new citrinin analogue, sargassopenilline $C$ (155), which possessed a unique 6,6-spiroketal skeleton and inhibited the transcription of oncogenic nuclear factor, AP-1 $\left(\mathrm{IC}_{50}=15 \mu \mathrm{M}\right)$ [104]. Two phenalenone-skeleton citrinin analogues, sculezonones A and B (156-157), isolated from a marine sponge-derived fungus Penicillium sp., inhibited both DNA polymerases ( $\alpha$ and $\beta$ ) [105]. Dicitrinone B (158), a marine sediment-derived fungal metabolite (P. citrinum) containing a rare carbon-bridge citrinin dimer, induced A-375 cell apoptosis by generating ROS via a caspase-related pathway [106]. In another study, two novel skeletal metabolites (159-160) possibly biogenetically derived from citrinin were found. Perinadine A (159), a scalusamide A-type pyrrolidine isolated from a fish gastrointestinal fungus, P. citrinum, exhibited mild cytotoxicity against a murine leukemia L1210 cell line $\left(\mathrm{IC}_{50}=20 \mu \mathrm{g} / \mathrm{mL}\right)$ [107]. However, herqueiazole (160), obtained from a marine sediment-derived fungus, Penicillium sp. F011, possessed a novel pyrrole-containing phenalenone moiety and demonstrated weak cytotoxicity against $\mathrm{A} 549$ cells $\left(\mathrm{IC}_{50}=67.3 \mu \mathrm{M}\right)$, while doxorubicin was used as a positive control with an $\mathrm{IC}_{50}$ value of $3.3 \mu \mathrm{M}$ [108]. 
<smiles>CC1=C2C(=CO[C@@H](C)[C@@H]2C)C(O)=C(C(=O)O)C1=O</smiles>

151<smiles>[R]c1c(O)c2c3c(c(O)c(OC)c(O)c3c1C)C(=O)C(O)(C(C)(C)C(C)=O)C2=O</smiles>

156: $\mathrm{R}=\mathrm{H}$

157: $\mathrm{R}=\mathrm{OH}$<smiles>[R]O[C@]1([2H])CCCC[C@]12O[C@H](C)[C@@H](C)c1c(C)c(C)c(O)c(C(=O)O)c12</smiles>

152: $\mathrm{R}=\mathrm{CH}_{3}$ 153: $\mathrm{R}=\mathrm{H}$

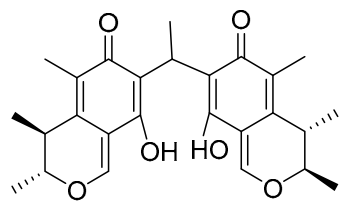

158<smiles>CCCCC[C@H]1Cc2cc(O)c(C(=O)O)c(O)c2C[C@H]1CC1OCC(CC(=O)C(C)(C)CC)[C@@H]1C</smiles>

154

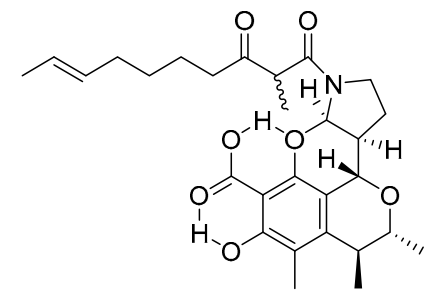

159

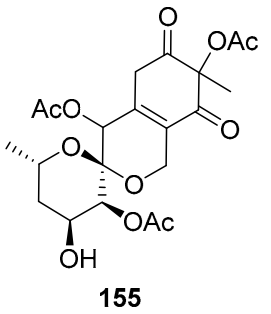<smiles>CC1=C2C(=O)C3(O)C(=C2c2c(oc4cc(C)[nH]c4c2=O)C(O)=C1)O[C@H](C)C3(C)C</smiles>

160

Figure 23. Chemical structures of compounds 151-160.

Other fungal azaphilone polyketides include comazaphilones D-F (161-163) (Figure 24), pinophilins A, B, and Sch 725680 (164-166), which were isolated from a marine sediment-derived fungus, P. commune QSD-17 (comazaphilones D-F), and a marine seaweed-derived P. pinophilum Hedgcok (pinophilins A-B and Sch 725680). Comazaphilones D-F (161-163) showed selective but weak cytotoxicity against SW1990 cell line ( $\mathrm{IC}_{50}=51,26$, and $53 \mu \mathrm{M}$, respectively), while fluoruoracil was used as a positive control with an $\mathrm{IC}_{50}$ value of $\left.120 \mu \mathrm{M}\right)$ [109]. Azaphilone derivatives (164-166) were suggested to suppress cancer cell proliferation by inhibiting DNA replication via the inhibition of mammalian DNA polymerases A, B, and Y [110].

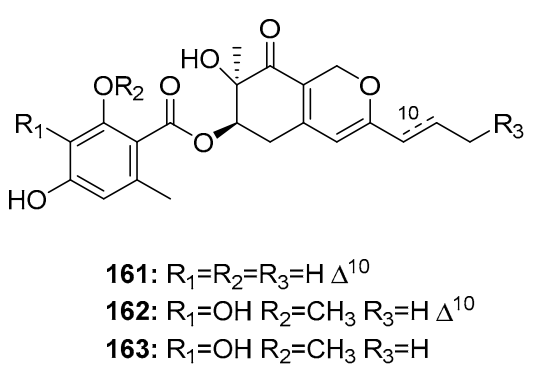

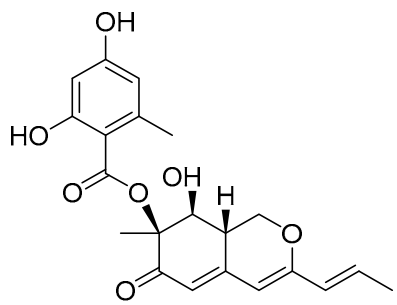

164

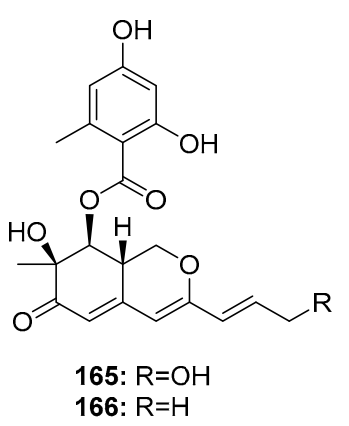

Figure 24. Chemical structures of compounds 161-166.

Penicillium sp. strain OUPS-79, which is derived from the marine alga Enteromorpha intestinalis, yielded various cytotoxic polyketides, including penostatins A-C, E-I (167-169, 171-175) (Figure 25) [111,112]. They were found to be significantly cytotoxic to P388 lymphocytic leukemia cells $\left(\mathrm{ED}_{50}=0.8,1.2,1.0,0.9,1.4,0.5,0.8\right.$, and $1.2 \mu \mathrm{g} / \mathrm{mL}$, respectively). However, penostatin $\mathrm{D}(\mathbf{1 7 0})$ exhibited moderate cytotoxicity $\left(\mathrm{ED}_{50}=11.0 \mu \mathrm{g} / \mathrm{mL}\right)$, which may be attributed to the absence of the cyclic conjugated enone system. Moreover, penostatin C (169) exhibited significant cytotoxicity in seven of the 36 cell lines tested with $\mathrm{ED}_{50}$ values ranging from 1 to $2 \mu \mathrm{g} / \mathrm{mL}$. Recent studies have shown that penostatins A-C (167-169) may be tyrosine phosphatase 1B (PTP1B) inhibitors, which can be used to treat type II diabetes and other associated metabolic diseases $\left(\mathrm{IC}_{50}=15.87,33.65\right.$, and $0.37 \mu \mathrm{M}$, respectively), while sodium orthovanadate was used as a positive control with an $\mathrm{IC}_{50}$ 
value of $0.65 \mu \mathrm{M}$ [113]. The total synthesis of penostatins $A, B$, and $F(167,168,172)$ was previously reported $[114,115]$.

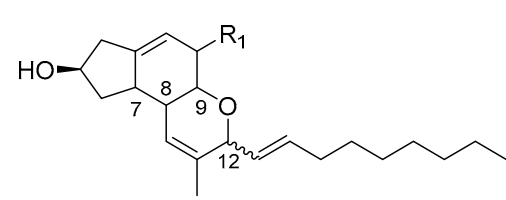

167: $\mathrm{R}_{1}==\mathrm{O}, 7 \beta \mathrm{H}, 8 \alpha \mathrm{H}, 9 \beta \mathrm{H}, 12 \beta$ 168: $R_{1}==0,7 \alpha H, 8 \beta H, 9 \alpha H, 12 \alpha$ 170: $\mathrm{R}_{1}=\alpha \mathrm{OH}, 7 \alpha \mathrm{H}, 8 \beta \mathrm{H}, 9 \alpha \mathrm{H}, 12 \alpha$

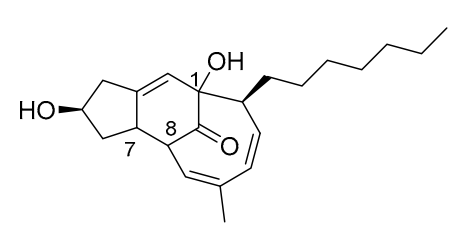

172: $1 \beta \mathrm{OH}, 7 \alpha \mathrm{H}, 8 \beta \mathrm{H}$ 175: $1 \alpha \mathrm{OH}, 7 \beta \mathrm{H}, 8 \alpha \mathrm{H}$

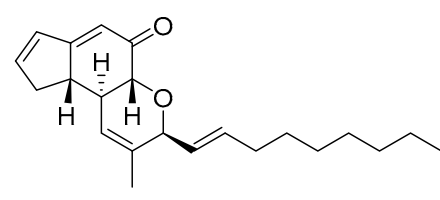

169

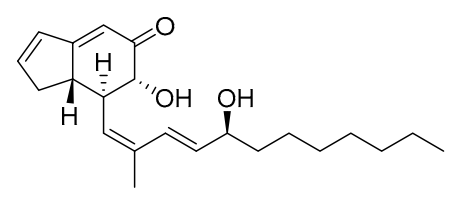

171

Figure 25. Chemical structures of compounds 167-174.

Fungal phenolic polyketides have diverse biological activities and unique structures [116]. A weak DNA topoisomerase I inhibitor, compound (176) (Figure 26), was obtained from the marine sediment-derived P. oxalicum HSY05 [117], whereas a racemic mixture (177-178) was obtained from the co-cultivation of marine mangrove-derived Penicillium sp. WC-29-5 and Streptomyces fradiae 007. Compounds 177-178 displayed significant cytotoxicity against H1975 cell lines $\left(\mathrm{IC}_{50}=3.97\right.$ and $5.73 \mu \mathrm{M}$, respectively). Moreover, compound 178 exhibited cytotoxicity against HL-60 cells $\left(\mathrm{IC}_{50}=3.73 \mu \mathrm{M}\right)$ [118]. Using a bioinformatics tool, Marine Halogenated Compound Analysis (MeHaloCoA), three halogenated bioactive metabolites, (+)-5-chlorogriseofulvin (179) as well as griseophenones I and G (180-181), were isolated from a marine-derived P. canescens. They inhibited the growth of $\mathrm{KB}$ cells at a concentration of $0.6 \mu \mathrm{M}(\mathrm{IR} \%=49,58$, and $47 \%$, respectively) [119]. Furthermore, one benzophenone, iso-monodictyphenone (182), and two diphenyl ether derivatives, penikellides A and B (183-184), were isolated from a mangrove endogenous fungus, Penicillium sp. MA-37. These three metabolites exhibited moderate brine shrimp lethality $\left(\operatorname{LD}_{50}=25.3\right.$, 14.2 , and $39.2 \mu \mathrm{M}$, respectively), while colchicine was used as a positive control with an $\mathrm{LD}_{50}$ value of $1.22 \mu \mathrm{M}$ [120]. Penicillide (185), a multifunctional metabolite produced by a marine sediment-derived Penicillium sp. strain, was shown to be an acyl-CoA cholesterol acyltransferase (ACAT) [121], nonpeptide calpain inhibitor [122], and oxytocin antagonist [123]. Furthermore, compound 185 was found to exhibit cytotoxic, antibiotic, and plant growth inhibitory properties. Recently, two marine fungi, P. pinophilum (derived from a gorgonian) and Penicillium sp. ZLN29 (derived from a sediment), were found to produce penicillide (185) and penicillide derivatives (186-187) that exhibited potent cytotoxicity against HepG2 cell line $\left(\mathrm{IC}_{50}=9.7\right.$ and $9.9 \mu \mathrm{M}$ for 185-186, respectively); moreover, compound 187 showed additional cytotoxicity against Hela cell line $\left(\mathrm{IC}_{50}=6.1 \mu \mathrm{M}\right)[124,125]$. Two anthraquinone derivatives, nidurufin (188) and averantin (189), isolated from a marine sediment-derived fungus, P. flavidorsum SHK1-27, were cytotoxic against K562 cell line $\left(\mathrm{IC}_{50}=12.6\right.$ and $27.7 \mu \mathrm{M}$, respectively), while adriamycin was used as a positive control with an $\mathrm{IC}_{50}$ value of $1.5 \mu \mathrm{M}$. Nidurufin (188) was suggested to induce cell cycle arrest at the G2/M transition in a time-dependent manner [126]. The total synthesis of $( \pm)$-nidurufin (188), an aflatoxin precursor, was previously described [127]. 


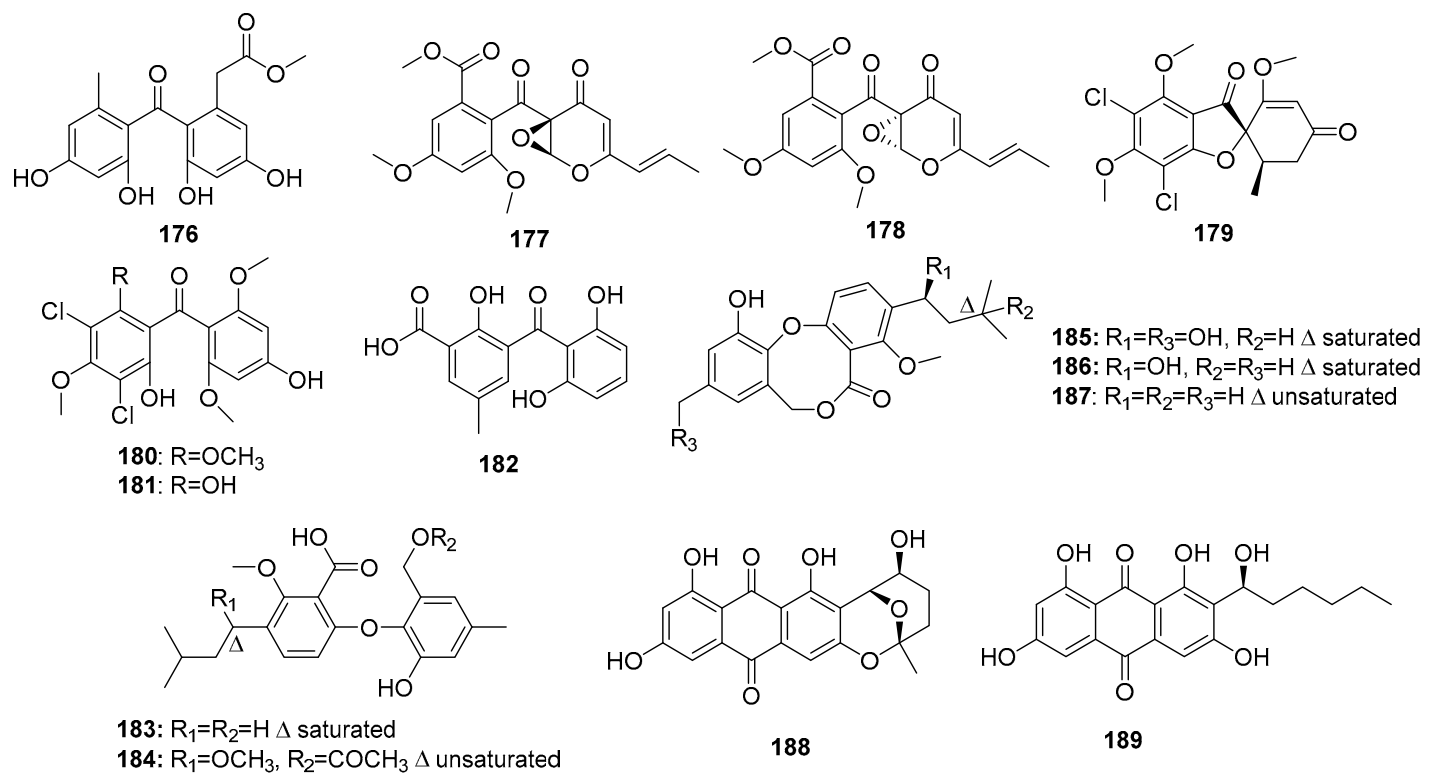

Figure 26. Chemical structures of compounds 176-189.

Members of the sorbicillinoid family are hexaketide metabolites isolated from various fungi. In 2005, Zhu et al. found two sorbicillin analogues (benzoquinone (190-191)), two bisvertinolones (192-193), and three bridged bicyclic bisorbicillinoids (194-196) (Figure 27) in a marine sediment-derived fungus, $P$. terrestre. Dihydrobisvertinolone (192) and trichodimerol (196) demonstrated the strongest cytotoxic effects $\left(\mathrm{IC}_{50}=0.52 \mu \mathrm{M}\right.$ in $\mathrm{A} 549, \mathrm{IC}_{50}=0.33 \mu \mathrm{M}$ in P388, respectively), while etoposide was used as a positive control with $\mathrm{IC}_{50}$ values of 1.4 and $0.064 \mu \mathrm{M}$, respectively $[128,129]$. The preliminary SAR showed that an intact sorbyl side chain played a decisive role [130]. Further investigation of this strain yielded two additional chlorinated sorbicillinoids (197-198). Interestingly, the configuration at C-19 was found to largely determine the cytotoxicity, wherein chloctanspirone A (197) (R configuration) was 4-fold more active than chloctanspirone B (198) (S configuration) in HL-60 and A549 cancer cell lines [131].<smiles>CC=CCCC(=O)C1=C(C)C(=O)C(O)=C(C)C1=O</smiles><smiles>CC(=O)CC1=C(C)C(=O)C(O)=C(C)C1=O</smiles>

191

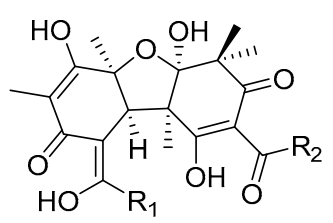

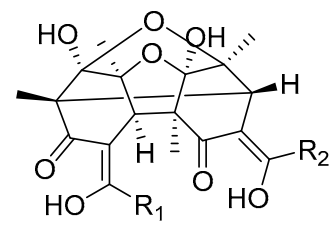

194: $\mathrm{R}_{1}=\mathrm{C}_{5} \mathrm{H}_{7}, \mathrm{R}_{2}=\mathrm{C}_{5} \mathrm{H}_{9}$ 192: $\mathrm{R}_{1}=\mathrm{C}_{5} \mathrm{H}_{7}, \mathrm{R}_{2}=\mathrm{C}_{5} \mathrm{H}_{9}$ 193: $\mathrm{R}_{1}=\mathrm{C}_{5} \mathrm{H}_{9}, \mathrm{R}_{2}=\mathrm{C}_{5} \mathrm{H}_{9}$ 195: $\mathrm{R}_{1}=\mathrm{C}_{5} \mathrm{H}_{9}, \mathrm{R}_{2}=\mathrm{C}_{5} \mathrm{H}_{9}$ 196: $\mathrm{R}_{1}=\mathrm{C}_{5} \mathrm{H}_{7}, \mathrm{R}_{2}=\mathrm{C}_{5} \mathrm{H}_{7}$<smiles>C/C=C/C=C/C(O)=C1/C(=O)C2(C)C(=O)C=C[C@]1(C(=O)O)[C@@H](O)[C@H](C)C=C2Cl</smiles>

197: $19 R$ 198: $19 S$

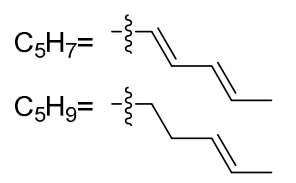

Figure 27. Chemical structures of compounds 190-198.

Macrolides represent a well-known class of antibiotics, and curvularin (200) (Figure 28) is a heat shock protein (HSP90) inhibitor [132]. (10E, 15S)-10,11-Dehydrocurvularin (199) was isolated from 
marine sponge-derived Penicillium sp. DRF2 and Curvularia sp. It exhibited significant cytotoxicity with mean $\mathrm{IC}_{50}$ values ranging from 0.28 to $6 \mu \mathrm{M}$ in 14 different solid tumors (36 tumor cell lines) $[133,134]$. Penicillium fungi are also a good source of tanzawaic acid polyketides, which exhibit antibiotic resistance [135], as well as anti-inflammatory [136] and cytotoxic activities. Tanzawaic acid P (201), isolated from a marine-derived fungus, Penicillium sp. CF07370, was selectively toxic to U937 cancer cells via the activation of the mitochondrial apoptotic pathway [137]. Computational ligand-protein-DNA binding analysis revealed that tanzawaic acid D (202), isolated from P. steckii, effectively and selectively bound to the transcription factor, forkhead box O1 (FOXO1), which can regulate epidermal growth factor receptor (EFGR) signaling, suppress cell cycle progression, and stabilize the conformation of FOXO1-DNA [138].<smiles>CCC(=O)OC(C)CCCCCC(=O)c1c(O)cc(O)cc1CC</smiles>

199: $\Delta$ unsaturated 200: $\Delta$ saturated

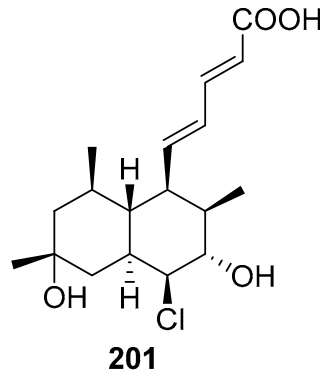

201<smiles>Cc1cc(O)c2c(c1/C=C/C=C/C(=O)O)C(C)CC(C)C2OC(=O)O</smiles>

Figure 28. Chemical structures of compounds 199-202.

\section{Lipopeptides}

Fellutamides A and B (210-211) (Figure 29) were the first cytotoxic lipopeptides isolated from fish-derived P. fellutanum [139]. Compounds $\mathbf{2 1 0}$ and 211 exhibited significant cytotoxicity against murine leukemia P388 $\left(\mathrm{IC}_{50}=0.2\right.$ and $0.1 \mu \mathrm{g} / \mathrm{mL}$, respectively), $\mathrm{L} 1210\left(\mathrm{IC}_{50}=0.8\right.$ and $0.7 \mu \mathrm{g} / \mathrm{mL}$, respectively), and $\mathrm{KB}$ cells $\left(\mathrm{IC}_{50}=0.5\right.$ and $0.7 \mu \mathrm{g} / \mathrm{mL}$, respectively). Recently, seven new similar lipopeptides, penicimutalides $A-G$ (203-209) and fellutamides B and C (211-212) were isolated from a diethyl sulfate-induced mutant of the marine fungus, P. purpurogenum G59 [140]. They were cytotoxic against five human cancer cell lines (K562, HL-60, Hela, BGC-823, and MCF-7). Compounds 203-209 and 212 exhibited weak cytotoxicity ( $\mathrm{IR} \%=10-50 \%$ at $100 \mu \mathrm{g} / \mathrm{mL}$, while 5 -fluoruoracil as a positive control with the IR\% of 37 50\% at $100 \mu \mathrm{g} / \mathrm{mL}$ ). However, fellutamide B (211) with a C-terminal aldehyde group was more potent with $\mathrm{IC}_{50}$ values that ranged from 20 to $80 \mu \mathrm{g} / \mathrm{mL}$, which indicated that the C-terminal aldehyde group improves the cytotoxicity.<smiles>[R]C(CC(C)C)NC(=O)C(CC(N)=O)NC(=O)C(CC(N)=O)NC(=O)CC(O)CCCCCCCCC</smiles>

203,204 $\mathrm{R}_{1}=\mathrm{H}, \mathrm{R}_{2}=\mathrm{OCH}_{2} \mathrm{CH}_{3}$, C-2 epimer

205,206 $\mathrm{R}_{1}=\mathrm{H}, \mathrm{R}_{2}=\mathrm{OCH}_{3}, \mathrm{C}-2$ epimer

$207 \mathrm{R}_{1}=\mathrm{H}, \mathrm{R}_{2}=\mathrm{CONH}_{2}, 2 \mathrm{~S}$

$208 \mathrm{R}_{1}=\mathrm{H}, \mathrm{R}_{2}=\mathrm{CONH}_{2}, 2 \mathrm{R}$

$210 \mathrm{R}_{1}=\mathrm{OH}, \mathrm{R}_{2}=\mathrm{CHO}, 2 \mathrm{~S}$

$211 \mathrm{R}_{1}=\mathrm{H}, \mathrm{R}_{2}=\mathrm{CHO}, 2 \mathrm{~S}$

$212 \mathrm{R}_{1}=\mathrm{H}, \mathrm{R}_{2}=\mathrm{CH}_{2} \mathrm{OH}, 2 \mathrm{~S}$<smiles>CCCCCCCCCC(O)CC(=O)NC(CC(N)O)C(=O)N[C@@H](CCC(N)=O)C(N)=O</smiles>

209

Figure 29. Chemical structures of compounds 203-212. 


\section{Miscellaneous Compounds}

Polyphenol derivatives are the most abundant fungal secondary metabolites. Unsurprisingly, marine Penicillium sp. is a good source of polyphenol derivatives. Two trimeric peniphenylanes A, B (213-214) and three dimeric peniphenylanes D, F, G (215-217) (Figure 30) were isolated from the deep sea sediment-derived fungus, P. fellutanum HDN14-323. Peniphenylane D (215) displayed more potent and extensive cytotoxicity with $\mathrm{IC}_{50}$ values in the range of $9-30 \mu \mathrm{M}$ in three cancer cell lines (Hela, HL-60, and HCT-116), while doxorubicin was used as a positive control with the $\mathrm{IC}_{50}$ values of $0.2,0.6$, and $0.2 \mu \mathrm{M}$, respectively [141]. The marine sediment-derived fungus, $P$. terrestre was found to produce several gentisyl alcohol derivatives, including trimeric terrestrol A (225) and dimeric terrestrols B-H (218-224), which were found to be cytotoxic against HL-60, MOLT-4, BEL-7402, and A549 cancer cell lines with $\mathrm{IC}_{50}$ values in the range of 5-65 $\mu \mathrm{M}$ [142]. Interestingly, the marine mangrove endogenous P. expansum 091006 yielded four novel cytotoxic phenolic bisabolane sesquiterpenoids (expansols A-C; $\mathrm{E}$ (226-229)) with $\mathrm{IC}_{50}$ values of $15.7,5.4,18.2$, and $20.8 \mu \mathrm{M}$, respectively, in HL-60 cells. In addition, expansol B (227) showed significant cytotoxicity against A549 cells $\left(\mathrm{IC}_{50}=1.9 \mu \mathrm{M}\right)$, while etoposide was used as a positive control with $\mathrm{IC}_{50}$ values of 0.042 and $0.63 \mu \mathrm{M}$ for two cell lines, respectively [143,144].<smiles>COCc1c(O)ccc(Cc2c(O)ccc(Cc3c(C)cccc3O)c2C)c1C</smiles>
213<smiles>Cc1cccc(O)c1COCc1c(O)ccc(Cc2c(C)cccc2O)c1C</smiles>

214<smiles>[R]c1cc(OCc2cc(O)ccc2O)cc(CO)c1O</smiles>
219: $\mathrm{R}=\mathrm{H}$

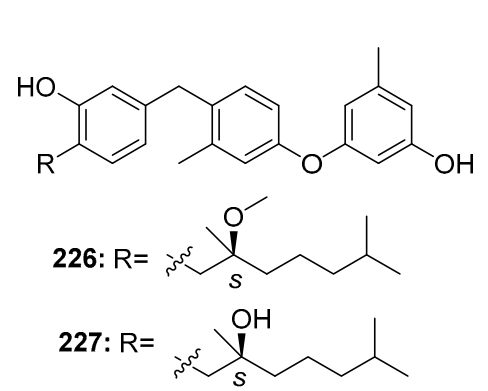
220: $\mathrm{R}=\mathrm{Cl}$ 221: $\mathrm{R}=\mathrm{H}$<smiles>C/C(=C/CCC(C)C)Cc1ccc(Cc2c(C)cc(Oc3cc(C)cc(O)c3)cc2O)cc1O</smiles><smiles>[R]Cc1c([R3])ccc(Cc2c(C)cccc2O)c1[R]</smiles>

215: $\mathrm{R}_{1}=\mathrm{CH}_{3}, \mathrm{R}_{2}=\mathrm{OCH}_{3}, \mathrm{R}_{3}=\mathrm{OH}$ 216: $\mathrm{R}_{1}=\mathrm{R}_{2}=\mathrm{OH}, \mathrm{R}_{3}=\mathrm{CH}_{3}$ 217: $\mathrm{R}_{1}=\mathrm{OH}, \mathrm{R}_{2}=\mathrm{OCH}_{3}, \mathrm{R}_{3}=\mathrm{CH}_{3}$<smiles>[R]c1cc(O)c(Cc2cc(O)ccc2O)c([R])c1O</smiles>
222: $\mathrm{R}_{1}=\mathrm{CH}_{2} \mathrm{OH}, \mathrm{R}_{2}=\mathrm{Cl}$ 223: $\mathrm{R}_{1}=\mathrm{Cl}, \mathrm{R}_{2}=\mathrm{H}$ 224: $\mathrm{R}_{1}=\mathrm{H}, \mathrm{R}_{2}=\mathrm{CH}_{3}$<smiles>COCc1cc(O)c(Cc2cc(O)c(Cc3cc(O)ccc3O)cc2O)cc1O</smiles>
225<smiles>CC(=CCCC(C)[18OH])Cc1ccc(Cc2c(O)cc(C)cc2Oc2cc(C)cc(O)c2)cc1O</smiles>

Figure 30. Chemical structures of compounds 213-229.

Patulin (230) (Figure 31) is a mycotoxin commonly found in rotting fruits, and is used as a potassium-uptake inhibitor or inducer of ion flux across cell membranes. An alga-derived Penicillium sp. was found to produce patulin (230) along with (+)-epiepoxydon (231), both of which exhibited extraordinary cytotoxic effects in P388 cells $\left(\mathrm{IC}_{50}=0.06\right.$ and $0.2 \mu \mathrm{g} / \mathrm{mL}$, respectively). Furthermore, (+)-epiepoxydon (231) had significant cytotoxicity against seven other cancer cell lines with $\mathrm{IC}_{50}$ values in the range of $0.3-1.5 \mu \mathrm{g} / \mathrm{mL}$ [111]. The isobenzofurannone derivative (232) isolated from a mangrove endophytic Penicillium sp. displayed moderate cytotoxicity against KB and KBV200 cells ( $\mathrm{IC}_{50}=6$ and $10 \mu \mathrm{g} / \mathrm{mL}$, respectively) [145], whereas the penicillic acid (233), isolated from marine-derived Penicillium strain, exhibited moderate cytotoxicity against POS1, AT6-1(murine prostatic carcinoma), and $\mathrm{L} 929$ (murine fibroblasts) cell lines ( $\mathrm{IC}_{50}=7.8,29.4$, and $12.9 \mu \mathrm{M}$, respectively) while doxorubicin was used as a positive control with $\mathrm{IC}_{50}$ values of $0.04 \sim 2 \mu \mathrm{M}$ [84]. 
<smiles>O=C1C=C2C(=CCOC2O)O1</smiles>

230<smiles>O=C1C(CO)=C[C@H](O)[C@H]2O[C@H]12</smiles>

231<smiles>COCc1cc(C)c(OC)c2c1CCC2=O</smiles>

232<smiles>C=C(C)C1(O)OC(=O)C=C1OC</smiles>

233

Figure 31. Chemical structures of compounds 230-233.

\section{Conclusions}

The rapid development of marine biotechnology and ever increasing needs of industrial applications resulted in the emergence of marine natural products as alternative drug sources in the early 1990s [146]. Marine-associated microorganisms are sensitive to culture conditions; therefore, strains living in extremely competitive environments tend to provide high potency leads (compound 154 in this review inhibited OVCAR-3 cell line at nanomolar concentrations). Furthermore, the activation of silent gene clusters may activate new biosynthetic pathways that produce compounds with novel structure, which provide equally valid leads (compounds 44 and 45, which have unique skeletons, had cytotoxic effects in the five cancer cell lines with $\mathrm{IC}_{50}$ values of $\sim 10 \mu \mathrm{M}$ ). Interestingly, the halogenation of compound 31, which was completely inactive, produces compound 32, which exhibited a much greater potency (compound 32 had significant cytotoxicity in 22Rv1 cells at nanomolar levels) [147].

The genus Penicillium has been explored for antitumor leads in recent years [148]. However, the marine ecological diversity of this genus offers more opportunities for drug discovery. This review includes more than 200 cytotoxic or antitumor compounds isolated from marine Penicillium fungus and chemically synthesized analogues. Of these, the major metabolites are alkaloids, particularly diketopiperazine alkaloids and indole alkaloids (Appendix A, Table A1). Cytochalasan alkaloids, which are indole alkaloids, constitute a large class of mycotoxins that exhibit significant cytotoxicity against P388 cells $\left(\mathrm{IC}_{50}<1 \mu \mathrm{g} / \mathrm{mL}\right)$. Furthermore, a series of diketopiperazine alkaloids, gliotoxin analogues, and roquefortine analogues with remarkable cytotoxicity at nanomolar levels are potential anticancer leads. Terpenoid metabolites appear to be more effective against cancer cell lines than steroids; in particular, compounds 99, 104, and 132 were effective at nanomolar levels. Furthermore, citrinins (chromone analogues) and their derivatives, which are polyketide mycotoxins, possess excellent cytotoxic activities. Penostatins (cytotoxic polyketides) are cytotoxic to P388 cells with $\mathrm{IC}_{50}$ values of $\sim 1 \mu \mathrm{g} / \mathrm{mL}$. With the exception of 210 and 211, lipopeptides exhibited moderate cytotoxicity. In addition, the Penicillium genus can produce polyphenolic compounds (terrestrols) with pronounced cytotoxicity.

Although our review includes most of the cytotoxic metabolites described in the literature, more compounds are yet to be identified in marine Penicillium sp. Different marine hosts and environments can also affect the biosynthesis of metabolites by endozoic fungi. Notably, over $99 \%$ of the symbiotic microorganisms cannot be cultured. Further investigations may utilize metagenome libraries of the host organisms to identify more metabolites produced by symbiotic microorganisms [149]. Additionally, further studies are needed to explore the functional mechanisms of the bioactive compounds and to optimize their production.

Acknowledgments: This research was a part of a project entitled 'Omics based fishery disease control technology development and industrialization' funded by the Korean Ministry of Oceans and Fisheries.

Author Contributions: Sen Liu and Jee H. Jung conceived and designed the study. Sen Liu collected and assessed the references. Sen Liu, Mingzhi Su, Shao-Jiang Song, and Jee H. Jung reviewed the manuscript. Sen Liu, and Jee H. Jung wrote the manuscript.

Conflicts of Interest: The authors have no conflict of interest to declare. 


\section{Appendix A}

Table A1. Secondary metabolites from Penicillium strain of marine origin. Items are listed according to the metabolite numbers used in this review.

\begin{tabular}{|c|c|c|c|c|c|c|c|}
\hline Metabolites & Producing Stain & $\begin{array}{l}\text { Environment } \\
\text { Source }\end{array}$ & Type & $\begin{array}{l}\text { Cell Lines/Brine } \\
\text { Shrimp }\end{array}$ & $\mathrm{IC}_{50}, \mathrm{LD}_{50}$, or IR (\%) & Target & References \\
\hline Penochalasin A (1) & Penicillium sp. & Marine alga & Indole alkaloid & P388 & $0.4 \mu \mathrm{g} / \mathrm{mL}$ & & [8] \\
\hline Penochalasin B (2) & Penicillium sp. & Marine alga & Indole alkaloid & P388 & $0.3 \mu \mathrm{g} / \mathrm{mL}$ & & [8] \\
\hline Penochalasin D (4) & Penicillium sp. & Marine alga & Indole alkaloid & P388 & $3.2 \mu \mathrm{g} / \mathrm{mL}$ & & [7] \\
\hline Penochalasin E (5) & Penicillium sp. & Marine alga & Indole alkaloid & P388 & $2.1 \mu \mathrm{g} / \mathrm{mL}$ & & [7] \\
\hline Penochalasin F (6) & Penicillium sp. & Marine alga & Indole alkaloid & P388 & $1.8 \mu \mathrm{g} / \mathrm{mL}$ & & [7] \\
\hline Penochalasin H (8) & Penicillium sp. & Marine alga & Indole alkaloid & P388 & $2.8 \mu \mathrm{g} / \mathrm{mL}$ & & [7] \\
\hline Penochalasin I (9) & $\begin{array}{l}\text { P. chrysogenum } \\
\text { V11 }\end{array}$ & Mangrove & Indole alkaloid & $\begin{array}{r}\text { MDA-MB-435, } \\
\text { SGC-7901, A549 }\end{array}$ & $(7.55,7.32,16.13) \mu \mathrm{M}$ & & [6] \\
\hline Penochalasin J (10) & $\begin{array}{l}\text { P. chrysogenum } \\
\text { V11 }\end{array}$ & Mangrove & Indole alkaloid & $\begin{array}{l}\text { MDA-MB-435, } \\
\text { SGC-7901, A549 }\end{array}$ & $(36.68,37.70,35.93) \mu \mathrm{M}$ & & [6] \\
\hline Chaetoglobosin A (11) & $\begin{array}{l}\text { P. chrysogenum } \\
\text { V11 }\end{array}$ & Mangrove & Indole alkaloid & $\begin{array}{l}\text { P388, MDA-MB-435, } \\
\text { SGC-7901, A549 }\end{array}$ & $\begin{array}{c}0.6 \mu \mathrm{g} / \mathrm{mL}(37.56,7.84 \\
6.56) \mu \mathrm{M}\end{array}$ & $\begin{array}{l}\text { Cell-cycle arrest } \\
\text { induction, } \\
\text { membrane ruffling } \\
\text { inhibition, and cell } \\
\text { migration }\end{array}$ & {$[6,8,9]$} \\
\hline Chaetoglobosin C (12) & $\begin{array}{l}\text { P. chrysogenum } \\
\text { V11 }\end{array}$ & Mangrove & Indole alkaloid & $\begin{array}{l}\text { MDA-MB-435, } \\
\text { SGC-7901, A549 }\end{array}$ & $(19.97,15.36,17.82) \mu \mathrm{M}$ & & [6] \\
\hline Chaetoglobosin E (13) & $\begin{array}{l}\text { P. chrysogenum } \\
\text { V11 }\end{array}$ & Mangrove & Indole alkaloid & A549 & $36.63 \mu \mathrm{M}$ & & [6] \\
\hline Chaetoglobosin F (14) & $\begin{array}{l}\text { P. chrysogenum } \\
\text { V11 }\end{array}$ & Mangrove & Indole alkaloid & $\begin{array}{l}\text { P388, MDA-MB-435, } \\
\text { SGC-7901, A549 }\end{array}$ & $\begin{array}{c}0.9 \mu \mathrm{g} / \mathrm{mL},(37.77,26.53 \\
27.72) \mu \mathrm{M}\end{array}$ & & {$[6,8]$} \\
\hline Chaetoglobosin G (15) & $\begin{array}{l}\text { P. chrysogenum } \\
\text { V11 }\end{array}$ & Mangrove & Indole alkaloid & $\begin{array}{l}\text { MDA-MB-435, } \\
\text { SGC-7901, A549 }\end{array}$ & $(38.77,25.86,27.63) \mu \mathrm{M}$ & & [6] \\
\hline Chaetoglobosin O (16) & Penicillium sp. & Marine alga & Indole alkaloid & P388 & $2.4 \mu \mathrm{g} / \mathrm{mL}$ & & [7] \\
\hline Cytoglobosin C (17) & $\begin{array}{l}\text { P. chrysogenum } \\
\text { V11 }\end{array}$ & Mangrove & Indole alkaloid & $\begin{array}{r}\text { MDA-MB-435, } \\
\text { SGC-7901, A549 }\end{array}$ & $(12.58,8.15,3.35) \mu \mathrm{M}$ & & [6] \\
\hline 18 & $\begin{array}{l}\text { Penicillium sp. } \\
\text { JMF034 }\end{array}$ & Deep sea sediment & Diketopiperazine & P388 & $3.4 \mu \mathrm{M}$ & & [12] \\
\hline
\end{tabular}


Table A1. Cont.

\begin{tabular}{|c|c|c|c|c|c|c|c|}
\hline Metabolites & Producing Stain & $\begin{array}{l}\text { Environment } \\
\text { Source }\end{array}$ & Type & $\begin{array}{l}\text { Cell Lines/Brine } \\
\text { Shrimp }\end{array}$ & $\mathrm{IC}_{50}, \mathrm{LD}_{50}$, or IR (\%) & Target & References \\
\hline 19 & $\begin{array}{l}\text { Penicillium sp. } \\
\text { JMF034 }\end{array}$ & Deep sea sediment & Diketopiperazine & P388 & $0.058 \mu \mathrm{M}$ & $\begin{array}{c}\text { HMT G9a } \\
\left(\mathrm{IC}_{50}=55 \mu \mathrm{M}\right)\end{array}$ & [12] \\
\hline 20 & $\begin{array}{l}\text { Penicillium sp. } \\
\text { JMF034 }\end{array}$ & Deep sea sediment & Diketopiperazine & P388 & $0.11 \mu \mathrm{M}$ & & [12] \\
\hline 21 & $\begin{array}{l}\text { Penicillium sp. } \\
\text { JMF034 }\end{array}$ & Deep sea sediment & Diketopiperazine & P388 & $0.11 \mu \mathrm{M}$ & $\begin{array}{c}\text { HMT G9a } \\
\left(\mathrm{IC}_{50}=58 \mu \mathrm{M}\right)\end{array}$ & [12] \\
\hline 22 & $\begin{array}{l}\text { Penicillium sp. } \\
\text { JMF034 }\end{array}$ & Deep sea sediment & Diketopiperazine & P388 & $0.056 \mu \mathrm{M}$ & $\begin{array}{c}\text { HMT G9a } \\
\left(\mathrm{IC}_{50}=2.6 \mu \mathrm{M}\right)\end{array}$ & [12] \\
\hline Gliotoxin (23) & $\begin{array}{l}\text { Penicillium sp. } \\
\text { JMF034 }\end{array}$ & Deep sea sediment & Diketopiperazine & P388 & $0.024 \mu \mathrm{M}$ & $\begin{array}{c}\text { HMT G9a } \\
\left(\mathrm{IC}_{50}=6.4 \mu \mathrm{M}\right) \\
\text { Dual inhibitor of } \\
\text { farnesyltransferase } \\
\text { and } \\
\text { geranylgeranyltransferase I }\end{array}$ & {$[12,13]$} \\
\hline Gliotoxin G (24) & $\begin{array}{c}\text { Penicillium sp. } \\
\text { JMF034 } \\
\text { P. brocae MA-231 }\end{array}$ & $\begin{array}{l}\text { Deep sea sediment } \\
\text { Mangrove }\end{array}$ & Diketopiperazine & $\begin{array}{l}\text { P388 A2780, A2780 } \\
\text { CisR }\end{array}$ & $\begin{array}{c}0.02 \mu \mathrm{M}(0.664 \\
0.661) \mu \mathrm{M}\end{array}$ & $\begin{array}{c}\text { HMT G9a } \\
\left(\mathrm{IC}_{50}=2.1 \mu \mathrm{M}\right)\end{array}$ & $\begin{array}{l}{[12]} \\
{[14]}\end{array}$ \\
\hline Brozazine A (25) & P. brocae MA-231 & Mangrove & Diketopiperazine & $\begin{array}{c}\text { Du145, Hela, HepG2, } \\
\text { MCF-7, NCI-H460, } \\
\text { SGC-7901, SW1990, } \\
\text { SW480, U251 }\end{array}$ & $\begin{array}{c}(4.2,6.8,6.4,5.5,4.9,2.6 \\
6.0,2.0,5.2) \mu \mathrm{M}\end{array}$ & & [16] \\
\hline Brozazine B (26) & P. brocae MA-231 & Mangrove & Diketopiperazine & $\begin{array}{l}\text { Du145, Hela, HepG2, } \\
\text { MCF-7, NCI-H460, } \\
\text { SGC-7901, SW1990, } \\
\text { SW480, U251 }\end{array}$ & $\begin{array}{c}(3.6,5.3,5.5,6.1,4.0,2.4 \\
\quad 6.4,1.2,3.5) \mu \mathrm{M}\end{array}$ & & [16] \\
\hline Brozazine E (29) & P. brocae MA-231 & Mangrove & Diketopiperazine & $\begin{array}{c}\text { Du145, Hela, HepG2, } \\
\text { MCF-7, NCI-H460, } \\
\text { SGC-7901, SW1990, } \\
\text { U251 }\end{array}$ & $\begin{array}{c}(11.2,4.3,5.6,9.0,12.4 \\
3.3,2.1,6.1) \mu \mathrm{M}\end{array}$ & & [16] \\
\hline Brozazine F (30) & P. brocae MA-231 & Mangrove & Diketopiperazine & $\begin{array}{c}\text { Du145, Hela, HepG2, } \\
\text { MCF-7, NCI-H460, } \\
\text { SGC-7901, SW1990, } \\
\text { U251 }\end{array}$ & $\begin{array}{c}(1.7,6.9,2.9,3.0,0.89 \\
\quad 8.0,5.9,5.3) \mu \mathrm{M}\end{array}$ & & [16] \\
\hline \multirow{2}{*}{$\begin{array}{l}N \text {-methylpretrichodermam } \\
\text { ide B/adametizines A (32) }\end{array}$} & \multirow{2}{*}{$\begin{array}{l}\text { P. adametzioides } \\
\text { AS-53 } \\
\text { Penicillium sp. }\end{array}$} & \multirow{2}{*}{$\begin{array}{l}\text { Marine } \\
\text { sponge/sediment/alga }\end{array}$} & \multirow{2}{*}{ Diketopiperazine } & Artemia salina & $4.8 \mu \mathrm{M}$ & & \multirow{2}{*}{ [17-19] } \\
\hline & & & & $\begin{array}{l}\text { L5178Y, 22Rv1, PC-3, } \\
\text { LNCaP }\end{array}$ & $(2,0.51,5.11,1.76) \mu \mathrm{M}$ & & \\
\hline
\end{tabular}


Table A1. Cont.

\begin{tabular}{|c|c|c|c|c|c|c|c|}
\hline Metabolites & Producing Stain & $\begin{array}{l}\text { Environment } \\
\text { Source }\end{array}$ & Type & $\begin{array}{l}\text { Cell Lines/Brine } \\
\text { Shrimp }\end{array}$ & $\mathrm{IC}_{50}, \mathrm{LD}_{50}$, or IR (\%) & Target & References \\
\hline Roquefortine C (33) & Penicillium sp. & Deep sea sediment & Diketopiperazine & & & $\begin{array}{c}\text { Activate } \\
P \text {-glycoprotein and } \\
\text { inhibit P450-3A and } \\
\text { other haemoproteins }\end{array}$ & {$[20,22]$} \\
\hline Roquefortine F (34) & Penicillium sp. & Deep sea sediment & Diketopiperazine & $\begin{array}{c}\text { A549, HL-60, } \\
\text { BEL-7402, MOLT-4 }\end{array}$ & $\begin{array}{c}(14.0,33.6,13.0 \\
21.2) \mu \mathrm{M}\end{array}$ & & [22] \\
\hline Roquefortine G (35) & Penicillium sp. & Deep sea sediment & Diketopiperazine & A549, HL-60 & $(42.5,36.6) \mu \mathrm{M}$ & & [22] \\
\hline Meleagrin (38) & $\begin{array}{l}\text { Penicillium sp. } \\
\text { P. commune } \\
\text { SD-118 }\end{array}$ & Deep sea sediment & Indole alkaloid & $\begin{array}{l}\text { A549, HL-60 HepG2, } \\
\text { NCI-H460, Hela, } \\
\text { DU145, MDA-MB-231, }\end{array}$ & $\begin{array}{c}(19.9,7.4) \mu \mathrm{M}(12.0,22.0 \\
20.0,11.0,5.0) \mu \mathrm{g} / \mathrm{mL}\end{array}$ & $\begin{array}{l}\text { Arrest the cell cycle } \\
\text { through } \mathrm{G}_{2} / \mathrm{M} \\
\text { phase } \\
\text { Inhibitor of tubulin } \\
\text { polymerization }\end{array}$ & [21-23] \\
\hline Meleagrin B (39) & Penicillium sp. & Deep sea sediment & Indole alkaloid & $\begin{array}{c}\text { A549, HL-60, } \\
\text { BEL-7402, MOLT-4 }\end{array}$ & $(2.7,6.7,1.8,2.9) \mu \mathrm{M}$ & & {$[21,22]$} \\
\hline Meleagrin C (40) & Penicillium sp. & Deep sea sediment & Indole alkaloid & $\begin{array}{l}\text { A549, BEL-7402, } \\
\text { MOLT-4 }\end{array}$ & $(9.9,10.0,4.7) \mu \mathrm{M}$ & & [22] \\
\hline Meleagrin D (41) & Penicillium sp. & Deep sea sediment & Indole alkaloid & A549 & $32.2 \mu \mathrm{M}$ & & [21] \\
\hline Penicimutanin (43) & $\begin{array}{l}\text { Mutant } P . \\
\text { purpurogenum } \\
\text { G59 }\end{array}$ & Marine soil & Diketopiperazine & K562, Hela, MCF-7 & $\begin{array}{c}\mathrm{IR} \%(100 \mu \mathrm{g} / \mathrm{mL}): \\
22.6 \%, 17.9 \%, 26.5 \%\end{array}$ & & [24] \\
\hline Penicimutanin A (44) & $\begin{array}{l}\text { Mutant } P . \\
\text { purpurogenum } \\
\text { G59 }\end{array}$ & Marine soil & Diketopiperazine & $\begin{array}{l}\text { K562, HL-60, Hela, } \\
\text { BGC-823, MCF-7 }\end{array}$ & $\begin{array}{c}(11.4,5.4,9.5,8.0,5.4) \\
\mu \mathrm{M}\end{array}$ & & [24] \\
\hline Penicimutanin B (45) & $\begin{array}{l}\text { Mutant } P . \\
\text { purpurogenum } \\
\text { G59 }\end{array}$ & Marine soil & Diketopiperazine & $\begin{array}{l}\text { K562, HL-60, Hela, } \\
\text { BGC-823, MCF-7 }\end{array}$ & $\begin{array}{c}(19.9,12.1,17.7,16.6, \\
8.0) \mu \mathrm{M}\end{array}$ & & [24] \\
\hline Fructigenine A (46) & $\begin{array}{l}\text { Mutant } P . \\
\text { purpurogenum } \\
\text { G59 }\end{array}$ & Marine soil & Diketopiperazine & $\begin{array}{l}\text { K562, Hela, MCF-7, } \\
\text { BGC-823 }\end{array}$ & $\begin{array}{c}\mathrm{IR} \%(100 \mu \mathrm{g} / \mathrm{mL}): 20.8 \% \\
\quad 55.3 \%, 65.6 \%, 34.8 \%\end{array}$ & & {$[24,25]$} \\
\hline $\begin{array}{c}11,11^{\prime} \text {-dideoxyverticillin } \mathrm{A} \\
(49)\end{array}$ & Penicillium sp. & Marine alga & Diketopiperazine & HCT-116 & $30 \mathrm{ng} / \mathrm{mL}$ & $\begin{array}{l}\text { Induce } \mathrm{G}_{2} / \mathrm{M} \text { arrest } \\
\text { through p38 MAPK } \\
\text { pathway; } \\
\text { Epidermal growth } \\
\text { factor receptor } \\
\text { tyrosine kinase } \\
\text { inhibitor }\end{array}$ & {$[28,30,31]$} \\
\hline
\end{tabular}


Table A1. Cont.

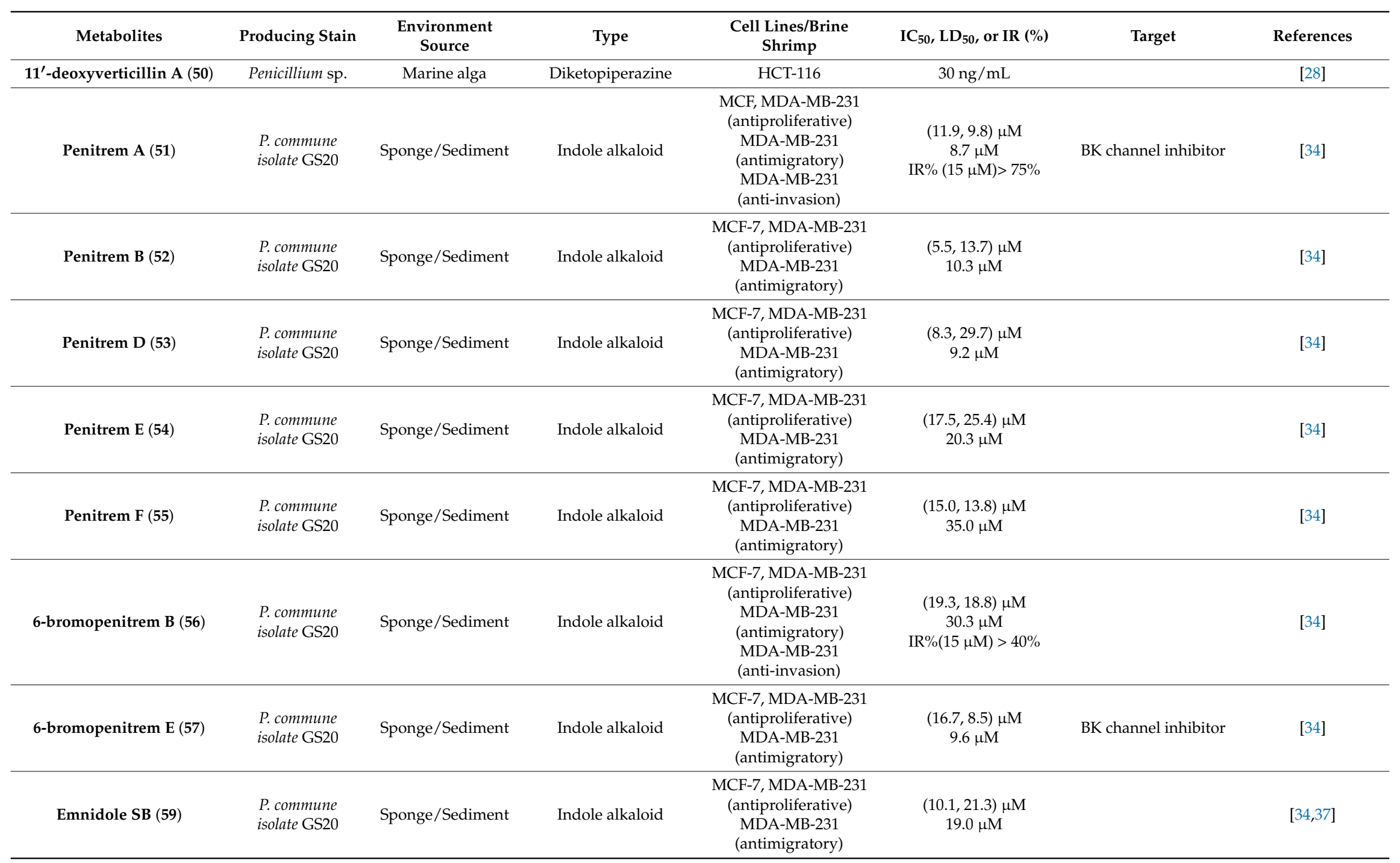


Table A1. Cont.

\begin{tabular}{|c|c|c|c|c|c|c|c|}
\hline Metabolites & Producing Stain & $\begin{array}{l}\text { Environment } \\
\text { Source }\end{array}$ & Type & $\begin{array}{l}\text { Cell Lines/Brine } \\
\text { Shrimp }\end{array}$ & $\mathrm{IC}_{50}, \mathrm{LD}_{50}$, or IR (\%) & Target & References \\
\hline Communesin A (60) & Penicillium sp. & $\begin{array}{c}\text { Marine } \\
\text { alga/Sediment }\end{array}$ & Indole alkaloid & P388 & $3.5 \mu \mathrm{g} / \mathrm{mL}$ & & {$[38,40]$} \\
\hline Communesin B (61) & Penicillium sp. & $\begin{array}{c}\text { Marine } \\
\text { alga/Sponge/Sedimen }\end{array}$ & ent Indole alkaloid & $\begin{array}{l}\text { P388, U-937, THP-1, } \\
\text { NAMALWA, MOLT-3, } \\
\text { SUP-B15 }\end{array}$ & $\begin{array}{c}(0.45,10.4,11.4,9.9,8.1 \\
7.2) \mu \mathrm{g} / \mathrm{mL}\end{array}$ & & {$[38-40]$} \\
\hline Communesin C (62) & Penicillium sp. & Marine sponge & Indole alkaloid & $\begin{array}{c}\text { U-937, THP-1, } \\
\text { NAMALWA, MOLT-3, } \\
\text { SUP-B15 }\end{array}$ & $\begin{array}{c}(11.3,13.1,8.2,8.6 \\
10.8) \mu \mathrm{g} / \mathrm{mL}\end{array}$ & & [39] \\
\hline Communesin D (63) & Penicillium sp. & Marine sponge & Indole alkaloid & $\begin{array}{c}\text { U-937, THP-1, } \\
\text { NAMALWA, MOLT-3, } \\
\text { SUP-B15 }\end{array}$ & $\begin{array}{c}(13.1,16.2,14.6,9.9 \\
9.0) \mu \mathrm{g} / \mathrm{mL}\end{array}$ & & [39] \\
\hline Penioxamide (64) & $\begin{array}{l}\text { P. oxalicum } \\
\text { EN-201 }\end{array}$ & Mangrove & Indole alkaloid & A. salina & $5.6 \mu \mathrm{M}$ & & [42] \\
\hline 65 & $\begin{array}{l}\text { P. brefeldianum } \\
\text { SD-273 }\end{array}$ & Marine sediment & Indole alkaloid & A. salina & $9.4 \mu \mathrm{M}$ & & [43] \\
\hline Penipaline B (66) & P. paneum SD-44 & Marine sediment & Indole alkaloid & A549, HCT-116 & $(20.44,14.88) \mu \mathrm{M}$ & & [44] \\
\hline Penipaline C (67) & P. paneum SD-44 & Marine sediment & Indole alkaloid & A549, HCT-116 & $(21.54,18.54) \mu \mathrm{M}$ & & [44] \\
\hline Terretrione C (69) & $\begin{array}{l}\text { Penicillium } \\
\text { sp.CYE-87 }\end{array}$ & Marine tunicate & 1,4-diazepane alkaloid & MDA-MB-231 & $17.6 \mu \mathrm{M}$ & & [46] \\
\hline Terretrione $\mathbf{D}(70)$ & $\begin{array}{l}\text { Penicillium } \\
\text { sp.CYE-87 }\end{array}$ & Marine tunicate & 1,4-diazepane alkaloid & MDA-MB-231 & $16.5 \mu \mathrm{M}$ & & [46] \\
\hline Penicillenol A1 (71) & $\begin{array}{l}\text { Penicillium sp. } \\
\text { GQ-7/P. citrinum }\end{array}$ & $\begin{array}{c}\text { Mangrove/Marine } \\
\text { sediment }\end{array}$ & Pyrrolidinone alkaloid & $\begin{array}{l}\text { A-375, HL-60, A549, } \\
\text { BEL-7402, P388 }\end{array}$ & $\begin{array}{c}3.2 \mu \mathrm{g} / \mathrm{mL}(0.76,23.8 \\
13.03,8.85) \mu \mathrm{M}\end{array}$ & & {$[47,49]$} \\
\hline Penicillenol A2 (72) & $\begin{array}{l}\text { Penicillium sp. } \\
\text { GQ-7/P. citrinum }\end{array}$ & $\begin{array}{c}\text { Mangrove/Marine } \\
\text { sediment }\end{array}$ & Pyrrolidinone alkaloid & $\begin{array}{l}\text { A-375 } \\
\text { HL-60 }\end{array}$ & $\begin{array}{c}13.8 \mu \mathrm{g} / \mathrm{mL} \\
16.26 \mu \mathrm{M}\end{array}$ & & {$[47,49]$} \\
\hline Penicillenol B1 (73) & $\begin{array}{l}\text { Penicillium sp. } \\
\text { GQ-7/P. citrinum }\end{array}$ & $\begin{array}{c}\text { Mangrove/Marine } \\
\text { sediment }\end{array}$ & Pyrrolidinone alkaloid & $\begin{array}{l}\text { A-375 } \\
\text { HL-60 }\end{array}$ & $\begin{array}{c}2.8 \mu \mathrm{g} / \mathrm{mL} \\
3.2 \mu \mathrm{M}\end{array}$ & & {$[47,49]$} \\
\hline Penicillenol B2 (74) & $\begin{array}{l}\text { Penicillium sp. } \\
\text { GQ-7/P. citrinum }\end{array}$ & $\begin{array}{c}\text { Mangrove/Marine } \\
\text { sediment }\end{array}$ & Pyrrolidinone alkaloid & $\begin{array}{l}\text { A-375 } \\
\text { HL-60 }\end{array}$ & $\begin{array}{c}0.97 \mu \mathrm{g} / \mathrm{mL} \\
7.65 \mu \mathrm{M}\end{array}$ & & {$[47,49]$} \\
\hline Penicillenol D1 (75) & P. citrinum & Marine sediment & Pyrrolidinone alkaloid & A549, HL-60 & $(17.2,18.5) \mu \mathrm{g} / \mathrm{mL}$ & & [48] \\
\hline Penicillenol D2 (76) & P. citrinum & Marine sediment & Pyrrolidinone alkaloid & A549, HL-60 & $(12.1,14.5) \mu \mathrm{g} / \mathrm{mL}$ & & [48] \\
\hline
\end{tabular}


Table A1. Cont.

\begin{tabular}{|c|c|c|c|c|c|c|c|}
\hline Metabolites & Producing Stain & $\begin{array}{l}\text { Environment } \\
\text { Source }\end{array}$ & Type & $\begin{array}{l}\text { Cell Lines/Brine } \\
\text { Shrimp }\end{array}$ & $\mathrm{IC}_{50}, \mathrm{LD}_{50}$, or IR (\%) & Target & References \\
\hline Penitrinine A (77) & P. citrinum & Marine sediment & Pyrrolidinone alkaloid & $\begin{array}{c}\text { A-375, SPC-A1, } \\
\text { HGC-27 }\end{array}$ & $(20.12,28.67,29.49) \mu \mathrm{M}$ & $\begin{array}{c}\text { Upregulate Bax, } \\
\text { downregulate Bcl-2, } \\
\text { suppress MMP-9 } \\
\text { and TIMP-1 }\end{array}$ & [50] \\
\hline 78 & P. janczewskii & Sea water & Quinolinone & $\begin{array}{c}\text { MDA-MB-231, } \\
\text { DU-145, SKOV-3, } \\
\text { HT-29, A549, CAKI-1, } \\
\text { SK-MEL-2, K562 } \\
\end{array}$ & $\begin{array}{c}\text { IR } \%(10 \mu \mathrm{g} / \mathrm{mL})= \\
20 \sim 50 \%\end{array}$ & & [52] \\
\hline 79 & P. janczewskii & Sea water & Quinolinone & $\begin{array}{c}\text { MDA-MB-231, } \\
\text { DU-145, SKOV-3, } \\
\text { HT-29, A549, CAKI-1, } \\
\text { SK-MEL-2, K562 }\end{array}$ & $\begin{array}{c}\text { IR } \%(10 \mu \mathrm{g} / \mathrm{mL})= \\
30 \sim 90 \%\end{array}$ & & [52] \\
\hline 80 & P. janczewskii & Sea water & Quinolinone & $\begin{array}{c}\text { MDA-MB-231, } \\
\text { DU-145, SKOV-3, } \\
\text { HT-29 }\end{array}$ & $\begin{array}{c}\text { IR } \%(10 \mu \mathrm{g} / \mathrm{mL})= \\
91.6 \%, 69.2 \%, 79.8 \%, \\
96.0 \%\end{array}$ & & [52] \\
\hline 81 & $\begin{array}{l}\text { Penicillium sp. } \\
\text { ghq208/Penicillium } \\
\text { sp. }\end{array}$ & $\begin{array}{c}\text { Marine } \\
\text { sediment/Mangrove }\end{array}$ & Quinolinone & 95-D, HepG2 & $(0.57,6.5) \mu \mathrm{g} / \mathrm{mL}$ & & {$[53,54]$} \\
\hline 82 & $\begin{array}{l}\text { Penicillium sp. } \\
\text { ghq208 }\end{array}$ & Marine sediment & Quinolinone & HepG2 & $13.2 \mu \mathrm{M}$ & & [53] \\
\hline 83 & $\begin{array}{l}\text { P. commune } \\
\text { SD-118 }\end{array}$ & Deep sea sediment & Quinazolinone & SW1990 & $20 \mu \mathrm{g} / \mathrm{mL}$ & & [23] \\
\hline 84 & $\begin{array}{l}\text { P. chrysogenum } \\
\text { EN-118 }\end{array}$ & Marine alga & Quinazolinone & DU145, A549, Hela & $8 \mu \mathrm{g} / \mathrm{mL}$ & & [56] \\
\hline 85 & $\begin{array}{l}\text { P. oxalicum } \\
\text { 0312F1 }\end{array}$ & Marine (not clear) & Quinazolinone & SGC-7901, BEL-7404 & $\begin{array}{c}\mathrm{IR} \%(200 \mu \mathrm{g} / \mathrm{mL})= \\
30 \sim 40 \%\end{array}$ & & [55] \\
\hline Penipacid A (86) & P. paneum SD-44 & Deep sea sediment & Amidine alkaloid & RKO & $8.4 \mu \mathrm{M}$ & & [57] \\
\hline Penipacid E (87) & P. paneum SD-44 & Deep sea sediment & Amidine alkaloid & RKO & $9.7 \mu \mathrm{M}$ & & [57] \\
\hline 88 & P. paneum SD-44 & Deep sea sediment & Imine alkaloid & Hela & $6.6 \mu \mathrm{M}$ & & [57] \\
\hline Penipanoid A (89) & P. paneum SD-44 & Deep sea sediment & Triazole alkaloid & SMMC-7721 & $54.2 \mu \mathrm{M}$ & & [58] \\
\hline Bis-sclerotioramin (90) & Penicillium 303\# & Mangrove & Azaphilone alkaloid & MDA-MB-231 & $7.13 \mu \mathrm{M}$ & & [59] \\
\hline Sorbicillactone (91) & P. chrysogenum & Marine sponge & $\begin{array}{l}\text { Miscellaneous } \\
\text { Alkaloid }\end{array}$ & L5178Y & $2.2 \mu \mathrm{g} / \mathrm{mL}$ & $\begin{array}{c}\text { Selective } \\
\text { anti-leukemic }\end{array}$ & [60] \\
\hline
\end{tabular}


Table A1. Cont.

\begin{tabular}{|c|c|c|c|c|c|c|c|}
\hline Metabolites & Producing Stain & $\begin{array}{l}\text { Environment } \\
\text { Source }\end{array}$ & Type & $\begin{array}{l}\text { Cell Lines/Brine } \\
\text { Shrimp }\end{array}$ & $\mathrm{IC}_{50}, \mathrm{LD}_{50}$, or IR $(\%)$ & Target & References \\
\hline Brocaeloid B (92) & P. brocae & Mangrove & $\begin{array}{l}\text { Miscellaneous } \\
\text { Alkaloid }\end{array}$ & A. salina & $36.7 \mu \mathrm{M}$ & & [62] \\
\hline Varitatin (93) & Mutant $P$. varibile & Mangrove & Amide alkaloid & HCT-116 & $2.8 \mu \mathrm{M}$ & $\begin{array}{c}\mathrm{IR} \%(1 \mu \mathrm{M})=50 \% \\
\text { and } 40 \% \\
(\mathrm{PDGF}-\beta \text { and } \\
\text { ErbB4) }\end{array}$ & [63] \\
\hline 18-hydroxydecaturin B (94) & $\begin{array}{l}\text { P. oxalicum } \\
\text { EN-201 }\end{array}$ & Mangrove & $\begin{array}{l}\text { Pyridinyl- } \alpha \text {-pyrone } \\
\text { alkaloid }\end{array}$ & A. salina & $2.3 \mu \mathrm{M}$ & & [42] \\
\hline Xantocillin X (95) & $\begin{array}{l}\text { P. commune } \\
\text { SD-118 }\end{array}$ & Deep sea sediment & Isocyanide alkaloid & $\begin{array}{c}\text { MCF-7, HepG2, } \\
\text { NCI-H460, Hela, } \\
\text { DU145, MDA-MB-231 }\end{array}$ & $\begin{array}{l}(12,7,10,10,8 \\
8) \mu \mathrm{g} / \mathrm{mL}\end{array}$ & $\begin{array}{c}\text { Inhibit MEK/EPK } \\
\text { pathway and } \\
\text { activate class III } \\
\text { PI3K/Beclin } 1 \\
\text { pathway }\end{array}$ & {$[23,67]$} \\
\hline 96 & $\begin{array}{l}\text { Penicillium sp. } \\
\text { PR19 N-1 }\end{array}$ & Marine sludge & Sesquiterpene & HL-60, A549 & $(45.8,82.8) \mu \mathrm{M}$ & & [70] \\
\hline 97 & $\begin{array}{l}\text { Penicillium sp. } \\
\text { PR19 N-1 }\end{array}$ & Marine sludge & Sesquiterpene & HL-60, A549 & $(28.3,5.2) \mu \mathrm{M}$ & & {$[70]$} \\
\hline 98 & $\begin{array}{l}\text { Penicillium sp. } \\
\text { PR19 N-1 }\end{array}$ & Marine sludge & Sesquiterpene & HL-60, A549 & $(11.8,12.2) \mu \mathrm{M}$ & & [71] \\
\hline 99 & $\begin{array}{l}\text { Penicillium sp. } \\
\quad \text { BL 27-2 }\end{array}$ & Sea mud & Sesquiterpene & $\begin{array}{l}\text { P388, A549, HL-60, } \\
\text { BEL-7402 }\end{array}$ & $\begin{array}{c}(0.073,0.096,0.065 \\
4.59) \mu \mathrm{M}\end{array}$ & & [72] \\
\hline Sporogen-AO 1 (100) & $\begin{array}{l}\text { Penicillium sp. } \\
\text { BL 27-2 }\end{array}$ & Sea mud & Sesquiterpene & $\begin{array}{l}\text { P388, A549, HL-60, } \\
\text { BEL-7402 }\end{array}$ & $(10.1,8.81,10.4,5.7) \mu \mathrm{M}$ & & [72] \\
\hline 101 & $\begin{array}{l}\text { Penicillium sp. } \\
\text { BL 27-2 }\end{array}$ & Sea mud & Sesquiterpene & $\begin{array}{l}\text { P388, A549, HL-60, } \\
\text { BEL-7402 }\end{array}$ & $\begin{array}{c}(8.71,3.51,7.75 \\
11.8) \mu \mathrm{M}\end{array}$ & & [72] \\
\hline Adametacorenol B (102) & $\begin{array}{c}\text { P. adametzioides } \\
\text { AS-53 }\end{array}$ & Marine sponge & Diterpene & NCI-H446 & $5.0 \mu \mathrm{M}$ & & [17] \\
\hline Conidiogenone B (103) & Penicillium sp. & Deep sea sediment & Diterpene & A549, HL-60 & $(40.3,28.2) \mu \mathrm{M}$ & & [22] \\
\hline Conidiogenone C (104) & Penicillium sp. & Deep sea sediment & Diterpene & HL-60, BEL-7402 & $(0.038,0.97) \mu \mathrm{M}$ & & [22] \\
\hline Conidiogenone D (105) & Penicillium sp. & Deep sea sediment & Diterpene & $\begin{array}{c}\text { A549, HL-60, } \\
\text { BEL-7402, MOLT-4 }\end{array}$ & $(9.3,5.3,11.7,21.1) \mu \mathrm{M}$ & & [22] \\
\hline Conidiogenone E (106) & Penicillium sp. & Deep sea sediment & Diterpene & A549, HL-60, MOLT-4 & $(15.1,8.5,25.8) \mu \mathrm{M}$ & & [22] \\
\hline Conidiogenone $F(107)$ & Penicillium sp. & Deep sea sediment & Diterpene & $\begin{array}{l}\text { A549, HL-60, } \\
\text { BEL-7402 }\end{array}$ & $(42.2,17.8,17.1) \mu \mathrm{M}$ & & [22] \\
\hline
\end{tabular}


Table A1. Cont.

\begin{tabular}{|c|c|c|c|c|c|c|c|}
\hline Metabolites & Producing Stain & $\begin{array}{l}\text { Environment } \\
\text { Source }\end{array}$ & Type & $\begin{array}{l}\text { Cell Lines/Brine } \\
\text { Shrimp }\end{array}$ & $\mathrm{IC}_{50}, \mathrm{LD}_{50}$, or IR (\%) & Target & References \\
\hline Conidiogenone G (108) & Penicillium sp. & Deep sea sediment & Diterpene & $\begin{array}{c}\text { A549, HL-60, } \\
\text { BEL-7402, MOLT-4 }\end{array}$ & $(8.3,1.1,43.2,4.7) \mu \mathrm{M}$ & & [22] \\
\hline Brevione I (109) & Penicillium sp. & Deep sea sediment & Diterpene & MCF-7 & $7.44 \mu \mathrm{M}$ & & [73] \\
\hline Brevione A (110) & Penicillium sp. & Deep sea sediment & Diterpene & MCF-7 & $28.4 \mu \mathrm{M}$ & & [73] \\
\hline Penicisteroid A (111) & $\begin{array}{l}\text { P. chrysogenum } \\
\text { QEN-24S }\end{array}$ & Marine alga & Steroid & $\begin{array}{l}\text { Hela, SW1990, } \\
\text { NCI-H460 }\end{array}$ & $(15,31,40) \mu \mathrm{g} / \mathrm{mL}$ & & [74] \\
\hline 112 & Penicillium sp. & Marine moss & Steroid & HepG2 & $10.4 \mu \mathrm{g} / \mathrm{mL}$ & & [75] \\
\hline 114 & Penicillium sp. & Marine moss & Steroid & HepG2 & $20.7 \mu \mathrm{g} / \mathrm{mL}$ & & [75] \\
\hline 115 & Penicillium sp. & Marine moss & Steroid & HepG2 & $16.8 \mu \mathrm{g} / \mathrm{mL}$ & & [75] \\
\hline 116 & Penicillium sp. & Marine moss & Steroid & HepG2 & $21.3 \mu \mathrm{g} / \mathrm{mL}$ & & [75] \\
\hline 117 & $\begin{array}{l}\text { P. stoloniferum } \\
\text { QY2-10 }\end{array}$ & Sea squirt & Steroid & P388 & $4.07 \mu \mathrm{M}$ & & {$[76]$} \\
\hline 118 & Penicillium sp. & Marine sponge & Steroid & K562 & $5.54 \mu \mathrm{M}$ & & {$[77]$} \\
\hline 119 & Penicillium sp. & Marine sponge & Steroid & K562 & $4.38 \mu \mathrm{M}$ & & [77] \\
\hline Dankasterone B (121) & Penicillium sp. & Marine sponge & Steroid & HL-60, Hela, K562 & $(3.25,4.74,7.89) \mu \mathrm{M}$ & & [77] \\
\hline $\begin{array}{l}\text { 7-deacetoxyyanuthone } \\
\text { (122) }\end{array}$ & Penicillium sp. & Marine (not clear) & Meroterpene & $\begin{array}{c}\text { A549, SKOV-3, } \\
\text { SKMEL-2, XF498, } \\
\text { HCT-15 }\end{array}$ & $\begin{array}{c}(7.74,6.35,3.86,10.04, \\
10.07) \mu \mathrm{g} / \mathrm{mL}\end{array}$ & & [79] \\
\hline Farnesylbenzenediol (123) & Penicillium sp. & Marine (not clear) & Meroterpene & $\begin{array}{c}\text { A549, SKOV-3, } \\
\text { SKMEL-2, XF498, } \\
\text { HCT-15 }\end{array}$ & $\begin{array}{c}(4.73,5.31,4.80,5.94 \\
6.11) \mu \mathrm{g} / \mathrm{mL}\end{array}$ & & [79] \\
\hline Farnesylquinone (124) & Penicillium sp. & Marine (not clear) & Meroterpene & $\begin{array}{c}\text { A549, SKOV-3, } \\
\text { SKMEL-2, XF498, } \\
\text { HCT-15 }\end{array}$ & $\begin{array}{c}(25.44,37.29,18.41 \\
38.07,42.56) \mu \mathrm{g} / \mathrm{mL}\end{array}$ & & [79] \\
\hline Penicillone A (125) & $\begin{array}{l}\text { Penicillium sp. } \\
\text { F11 }\end{array}$ & Marine (not clear) & Meroterpene & HT1080, Cne2 & $(45.8,46.2) \mu \mathrm{M}$ & & [80] \\
\hline Phenylpyropene E (126) & $\begin{array}{l}\text { P. concentricum } \\
\text { ZLQ-69 }\end{array}$ & Sea water & Sesquiterpene & MGC-803 & $19.1 \mu \mathrm{M}$ & & [81] \\
\hline
\end{tabular}


Table A1. Cont.

\begin{tabular}{|c|c|c|c|c|c|c|c|}
\hline Metabolites & Producing Stain & $\begin{array}{l}\text { Environment } \\
\text { Source }\end{array}$ & Type & $\begin{array}{l}\text { Cell Lines/Brine } \\
\text { Shrimp }\end{array}$ & $\mathrm{IC}_{50}, \mathrm{LD}_{50}$, or IR $(\%)$ & Target & References \\
\hline Phenylpyropene F (127) & $\begin{array}{l}\text { P. concentricum } \\
\text { ZLQ-69 }\end{array}$ & Sea water & Sesquiterpene & MGC-803 & $13.6 \mu \mathrm{M}$ & & {$[81]$} \\
\hline Penicillipyrone A (128) & $\begin{array}{l}\text { Penicillium sp. } \\
\text { F446 }\end{array}$ & Marine sediment & Sesquiterpene & K562, A549 & $(28,15) \mu \mathrm{M}$ & & [82] \\
\hline Penicillipyrone B (129) & $\begin{array}{l}\text { Penicillium sp. } \\
\text { F446 }\end{array}$ & Marine sediment & Sesquiterpene & K562, A549 & $(50,17) \mu \mathrm{M}$ & & [82] \\
\hline 130 & Penicillium 303\# & Mangrove & Meroterpene & $\begin{array}{c}\text { MDA-MB-435, HepG2, } \\
\text { HCT-116, A549 }\end{array}$ & $\begin{array}{c}(34.25,24.56,33.72 \\
37.82) \mu \mathrm{g} / \mathrm{mL}\end{array}$ & & [59] \\
\hline 131 & Penicillium 303\# & Mangrove & Meroterpene & $\begin{array}{c}\text { MDA-MB-435, HepG2, } \\
\text { HCT-116, A549 }\end{array}$ & $\begin{array}{c}(31.32,23.87,29.19 \\
34.06) \mu \mathrm{g} / \mathrm{mL}\end{array}$ & & [59] \\
\hline Ligerin (132) & Penicillium sp. & Sea water & Merosesquiterpene & POS1, SaOS2, MG63 & $(117 / 78,137,1459) \mathrm{nM}$ & & {$[84,85]$} \\
\hline Oxalicumone D (133) & $\begin{array}{l}\text { P. oxalicum } \\
\text { SCSGAF } 0023\end{array}$ & Marine gorgonian & Chromone & BGC823, MOLT-4 & $(10.10,5.74) \mu \mathrm{M}$ & & [87] \\
\hline Oxalicumone E (134) & $\begin{array}{c}\text { P. oxalicum } \\
\text { SCSGAF } 0023\end{array}$ & Marine gorgonian & Chromone & $\begin{array}{c}\text { H1975, U937, K5652, } \\
\text { BGC823, MOLT-4, } \\
\text { MCF-7, HL-60, Huh-7 }\end{array}$ & $\begin{array}{c}(5.45,4.16,8.80,1.96 \\
1.36,4.32,2.96,6.33) \mu \mathrm{M}\end{array}$ & & [87] \\
\hline Oxalicumone A (135) & $\begin{array}{c}\text { P. oxalicum } \\
\text { SCSGAF } 0023\end{array}$ & Marine gorgonian & Chromone & $\begin{array}{c}\text { H1975, U937, K5652, } \\
\text { BGC823, MOLT-4, } \\
\text { MCF-7, HL-60, Huh-7, } \\
\text { A375, A549, Hela, } \\
\text { HepG2, SW-620, L-02 }\end{array}$ & $\begin{array}{c}(10.38,2.35,4.53,4.89 \\
0.30,11.30,2.55,9.49 \\
11.7,41.9,46.2,77.8,22.6 \\
99.0) \mu \mathrm{M}\end{array}$ & & {$[87,88]$} \\
\hline Oxalicumone B (136) & $\begin{array}{c}\text { P. oxalicum } \\
\text { SCSGAF } 0023\end{array}$ & Marine gorgonian & Chromone & $\begin{array}{l}\text { U937, MOLT-4, HL-60, } \\
\text { A375, Hela, SW-620 }\end{array}$ & $\begin{array}{c}(5.00,2.30,6.41,27.8, \\
60.9,40.6) \mu \mathrm{M}\end{array}$ & & {$[87,88]$} \\
\hline Chromosulfine (145) & $\begin{array}{c}\text { Mutant } P . \\
\text { purpurogenum } \\
\text { G59 }\end{array}$ & Marine (not clear) & Chromone & $\begin{array}{l}\text { K562, HL-60, BGC-823, } \\
\text { Hela, MCF-7 }\end{array}$ & $\begin{array}{c}(60.8,16.7,73.8,75.4 \\
59.2) \mu \mathrm{M}\end{array}$ & & [91] \\
\hline Secalonic acid F (146) & Penicillium sp. & Deep sea sediment & Xanthone & HL-60 & & $\begin{array}{l}\text { Modulate Rho GDP } \\
\text { dissociation } \\
\text { inhibitor } 2 \text { Activate } \\
\text { caspase } 3 \text { and } \\
\text { caspase } 9\end{array}$ & {$[92,93]$} \\
\hline Penimethavone A (147) & P. chrysogenum & Marine gorgonian & Flavone & $\begin{array}{c}\text { Hela, } \\
\text { rhabdomyosarcoma }\end{array}$ & $(8.41,8.18) \mu \mathrm{M}$ & & [94] \\
\hline Bipenicillisorin (148) & $\begin{array}{l}\text { P. chrysogenum } \\
\text { SCSIO } 41001\end{array}$ & Deep sea sediment & Coumarin & K562, A549, Huh-7 & $(6.78,6.94,2.59) \mu \mathrm{M}$ & & [95] \\
\hline
\end{tabular}


Table A1. Cont.

\begin{tabular}{|c|c|c|c|c|c|c|c|}
\hline Metabolites & Producing Stain & $\begin{array}{l}\text { Environment } \\
\text { Source }\end{array}$ & Type & $\begin{array}{l}\text { Cell Lines/Brine } \\
\text { Shrimp }\end{array}$ & $\mathrm{IC}_{50}, \mathrm{LD}_{50}$, or IR $(\%)$ & Target & References \\
\hline Monocerin (149) & Penicillium sp. & Marine sponge & Coumarin & L5178Y & $8.4 \mu \mathrm{M}$ & & [96] \\
\hline 150 & $\begin{array}{l}\text { Penicillium sp. } \\
\text { ZH16 }\end{array}$ & Mangrove & Coumarin & KB, KBv200 & $(5,10) \mu \mathrm{g} / \mathrm{mL}$ & & [97] \\
\hline Citrinin (151) & $\begin{array}{l}\text { Penicillium sp } \\
\text { FF001 }\end{array}$ & Marine sponge & $\begin{array}{l}\text { Azaphilone } \\
\text { polyketide }\end{array}$ & & & $\begin{array}{l}\text { Inhibit respiration } \\
\text { complex III }\end{array}$ & {$[99,101]$} \\
\hline Penicitrinol L (152) & P. citrinum & Marine sediment & $\begin{array}{l}\text { Azaphilone } \\
\text { polyketide }\end{array}$ & SW-620 & $25.6 \mu \mathrm{M}$ & & [48] \\
\hline Berkelic acid (154) & Penicillium sp. & Acid mine lake & $\begin{array}{l}\text { Azaphilone } \\
\text { polyketide }\end{array}$ & OVCAR-3 (in NCI60) & $91 \mathrm{nM}$ & $\begin{array}{c}\text { Inhibit MMP-3 }\left(\mathrm{GI}_{50}\right. \\
=1.87 \mu \mathrm{M}) \text { Inhibit } \\
\text { caspase } 1 \\
\left(\mathrm{GI}_{50}=98 \mu \mathrm{M}\right)\end{array}$ & [102] \\
\hline Sargassopenilline C (155) & P. thomii & Marine alga & $\begin{array}{l}\text { Azaphilone } \\
\text { polyketide }\end{array}$ & & & $\begin{array}{c}\text { Inhibit the } \\
\text { oncogenic nuclear } \\
\text { factor AP-1 } \\
\left(\mathrm{IC}_{50}=15 \mu \mathrm{M}\right)\end{array}$ & [104] \\
\hline Sculezonone B (157) & Penicillium sp. & Marine sponge & $\begin{array}{l}\text { Azaphilone } \\
\text { polyketide }\end{array}$ & & & $\begin{array}{l}\text { Inhibit both DNA } \\
\text { polymerases }(\alpha \\
\text { and } \beta)\end{array}$ & [105] \\
\hline Dicitrinone B (158) & P. citrinum & Marine sediment & $\begin{array}{l}\text { Azaphilone } \\
\text { polyketide }\end{array}$ & & & $\begin{array}{l}\text { Induce apoptosis } \\
\text { through ROS-related } \\
\text { caspase pathway }\end{array}$ & [106] \\
\hline Perinadine A (159) & P. citrinum & Marine fish & $\begin{array}{l}\text { Azaphilone } \\
\text { polyketide }\end{array}$ & L1210 & $20 \mu \mathrm{g} / \mathrm{mL}$ & & [107] \\
\hline Herqueiazole (160) & $\begin{array}{l}\text { Penicillium sp } \\
\text { F011 }\end{array}$ & Marine sediment & $\begin{array}{l}\text { Azaphilone } \\
\text { polyketide }\end{array}$ & A549 & $67.3 \mu \mathrm{M}$ & & {$[108]$} \\
\hline Comazaphilone D (161) & $\begin{array}{l}\text { P. commune } \\
\text { QSD-17 }\end{array}$ & Marine sediment & $\begin{array}{l}\text { Azaphilone } \\
\text { polyketide }\end{array}$ & SW1990 & $51 \mu \mathrm{M}$ & & [109] \\
\hline Comazaphilone E (162) & $\begin{array}{l}\text { P. commune } \\
\text { QSD-17 }\end{array}$ & Marine sediment & $\begin{array}{l}\text { Azaphilone } \\
\text { polyketide }\end{array}$ & SW1990 & $26 \mu \mathrm{M}$ & & [109] \\
\hline
\end{tabular}


Table A1. Cont.

\begin{tabular}{|c|c|c|c|c|c|c|c|}
\hline Metabolites & Producing Stain & $\begin{array}{l}\text { Environment } \\
\text { Source }\end{array}$ & Type & $\begin{array}{l}\text { Cell Lines/Brine } \\
\text { Shrimp }\end{array}$ & $\mathrm{IC}_{50}, \mathrm{LD}_{50}$, or IR (\%) & Target & References \\
\hline Comazaphilone F (163) & $\begin{array}{l}\text { P. commune } \\
\text { QSD-17 }\end{array}$ & Marine sediment & $\begin{array}{l}\text { Azaphilone } \\
\text { polyketide }\end{array}$ & SW1990 & $53 \mu \mathrm{M}$ & & [109] \\
\hline Pinophilin A (164) & $\begin{array}{l}\text { P. pinophilum } \\
\text { Hedgcok }\end{array}$ & Marine seaweed & $\begin{array}{l}\text { Azaphilone } \\
\text { polyketide }\end{array}$ & $\begin{array}{c}\text { A549, BALL-1, } \\
\text { HCT-116, Hela, } \\
\text { NUGC-3 }\end{array}$ & $\begin{array}{c}(52.5,50.2,51.3,55.6 \\
54.7) \mu \mathrm{M}\end{array}$ & $\begin{array}{c}\text { Inhibit the } \\
\text { mammalian DNA } \\
\text { polymerases A, B, Y } \\
\text { family }\end{array}$ & [110] \\
\hline Pinophilin B (165) & $\begin{array}{l}\text { P. pinophilum } \\
\text { Hedgcok }\end{array}$ & Marine seaweed & $\begin{array}{l}\text { Azaphilone } \\
\text { polyketide }\end{array}$ & $\begin{array}{l}\text { A549, BALL-1, } \\
\text { HCT-116, Hela, } \\
\text { NUGC-3 }\end{array}$ & $\begin{array}{c}(93.1,90.4,92.5,99.0 \\
96.8) \mu \mathrm{M}\end{array}$ & $\begin{array}{c}\text { Inhibit the } \\
\text { mammalian DNA } \\
\text { polymerases A, B, Y } \\
\text { family }\end{array}$ & [110] \\
\hline Sch $725680(166)$ & $\begin{array}{l}\text { P. pinophilum } \\
\text { Hedgcok }\end{array}$ & Marine seaweed & $\begin{array}{l}\text { Azaphilone } \\
\text { polyketide }\end{array}$ & $\begin{array}{c}\text { A549, BALL-1, } \\
\text { HCT-116, Hela, } \\
\text { NUGC-3 }\end{array}$ & $\begin{array}{c}(65.7,62.0,64.6,68.8 \\
66.4) \mu \mathrm{M}\end{array}$ & $\begin{array}{c}\text { Inhibit the } \\
\text { mammalian DNA } \\
\text { polymerases A, B, Y } \\
\text { family }\end{array}$ & [110] \\
\hline Penostatin A (167) & $\begin{array}{l}\text { Penicillium sp. } \\
\text { OUPS-79 }\end{array}$ & Marine alga & Polyketide & P388 & $0.8 \mu \mathrm{g} / \mathrm{mL}$ & $\begin{array}{l}\text { PTP1B inhibitor } \\
\left(\mathrm{IC}_{50}=15.87 \mu \mathrm{M}\right)\end{array}$ & {$[111,113]$} \\
\hline Penostatin B (168) & $\begin{array}{l}\text { Penicillium sp. } \\
\text { OUPS-79 }\end{array}$ & Marine alga & Polyketide & P388 & $1.2 \mu \mathrm{g} / \mathrm{mL}$ & $\begin{array}{l}\text { PTP1B inhibitor } \\
\left(\mathrm{IC}_{50}=33.65 \mu \mathrm{M}\right)\end{array}$ & {$[111,113]$} \\
\hline Penostatin D (170) & $\begin{array}{l}\text { Penicillium sp. } \\
\text { OUPS-79 }\end{array}$ & Marine alga & Polyketide & P388 & $11.0 \mu \mathrm{g} / \mathrm{mL}$ & & [111] \\
\hline Penostatin E (171) & $\begin{array}{l}\text { Penicillium sp. } \\
\text { OUPS-79 }\end{array}$ & Marine alga & Polyketide & P388 & $0.9 \mu \mathrm{g} / \mathrm{mL}$ & & [111] \\
\hline Penostatin F (172) & $\begin{array}{l}\text { Penicillium sp. } \\
\text { OUPS-79 }\end{array}$ & Marine alga & Polyketide & P388 & $1.4 \mu \mathrm{g} / \mathrm{mL}$ & & [112] \\
\hline Penostatin G (173) & $\begin{array}{l}\text { Penicillium sp. } \\
\text { OUPS-79 }\end{array}$ & Marine alga & Polyketide & P388 & $0.5 \mu \mathrm{g} / \mathrm{mL}$ & & [112] \\
\hline Penostatin H (174) & $\begin{array}{l}\text { Penicillium sp. } \\
\text { OUPS-79 }\end{array}$ & Marine alga & Polyketide & P388 & $0.8 \mu \mathrm{g} / \mathrm{mL}$ & & [112] \\
\hline Penostatin I (175) & $\begin{array}{l}\text { Penicillium sp. } \\
\text { OUPS-79 }\end{array}$ & Marine alga & Polyketide & P388 & $1.2 \mu \mathrm{g} / \mathrm{mL}$ & & [112] \\
\hline
\end{tabular}


Table A1. Cont.

\begin{tabular}{|c|c|c|c|c|c|c|c|}
\hline Metabolites & Producing Stain & $\begin{array}{l}\text { Environment } \\
\text { Source }\end{array}$ & Type & $\begin{array}{l}\text { Cell Lines/Brine } \\
\text { Shrimp }\end{array}$ & $\mathrm{IC}_{50}, \mathrm{LD}_{50}$, or IR (\%) & Target & References \\
\hline 176 & $\begin{array}{l}\text { P. oxalicum } \\
\text { HSY05 }\end{array}$ & Marine sediment & Phenolic polyketide & & & $\begin{array}{l}\text { DNA topoisomerase } \\
\text { I inhibitor }\end{array}$ & [117] \\
\hline 177 & $\begin{array}{l}\text { Co-cultured } \\
\text { Penicillium sp. } \\
\text { WC-29-5 }\end{array}$ & Mangrove & Phenolic polyketide & H1975 & $3.97 \mu \mathrm{M}$ & & [118] \\
\hline 178 & $\begin{array}{l}\text { Co-cultured } \\
\text { Penicillium sp. } \\
\text { WC-29-5 }\end{array}$ & Mangrove & Phenolic polyketide & H1975, HL-60 & $(5.73,3.73) \mu \mathrm{M}$ & & [118] \\
\hline $\begin{array}{c}\text { (+)-5-chlorogriseofulvin } \\
\text { (179) }\end{array}$ & $\begin{array}{l}\text { P. canescens } \\
\text { MMS460 }\end{array}$ & Sea water & Phenolic polyketide & $\mathrm{KB}$ & $\mathrm{IR} \%(0.6 \mu \mathrm{M})=49 \%$ & & [119] \\
\hline Griseophenone I (180) & $\begin{array}{l}\text { P. canescens } \\
\text { MMS460 }\end{array}$ & Sea water & Phenolic polyketide & КВ & $\mathrm{IR} \%(0.6 \mu \mathrm{M})=58 \%$ & & [119] \\
\hline Griseophenone G (181) & $\begin{array}{l}\text { P. canescens } \\
\text { MMS460 }\end{array}$ & Sea water & Phenolic polyketide & $\mathrm{KB}$ & $\mathrm{IR} \%(0.6 \mu \mathrm{M})=47 \%$ & & [119] \\
\hline $\begin{array}{l}\text { Iso-monodictyphenone } \\
\text { (182) }\end{array}$ & $\begin{array}{l}\text { Penicillium sp. } \\
\text { MA-37 }\end{array}$ & Mangrove & Phenolic polyketide & A. salina & $25.3 \mu \mathrm{M}$ & & [120] \\
\hline Penikellide A (183) & $\begin{array}{l}\text { Penicillium sp. } \\
\text { MA-37 }\end{array}$ & Mangrove & Phenolic polyketide & A. salina & $14.2 \mu \mathrm{M}$ & & [120] \\
\hline Penikellide B (184) & $\begin{array}{l}\text { Penicillium sp. } \\
\text { MA-37 }\end{array}$ & Mangrove & Phenolic polyketide & A. salina & $39.2 \mu \mathrm{M}$ & & [120] \\
\hline Penicillide (185) & $\begin{array}{l}\text { Penicillium sp. } \\
\text { ZLN29 }\end{array}$ & Marine sediment & Phenolic polyketide & HepG2 & $(6.7 / 9.7,7.8) \mu \mathrm{M}$ & $\begin{array}{c}\text { ACAT and } \\
\text { nonpeptide calpain } \\
\text { inhibitor }\end{array}$ & {$[121,122,124,125]$} \\
\hline Prepenicillide (186) & $\begin{array}{l}\text { Penicillium sp. } \\
\text { ZLN29 }\end{array}$ & Marine sediment & Phenolic polyketide & HepG2, RD & $9.9 \mu \mathrm{M}$ & & [124] \\
\hline Hydroxypenicillide (187) & P. pinophilum & Marine gorgonian & Phenolic polyketide & Hela & $6.1 \mu \mathrm{M}$ & & [125] \\
\hline Nidurufin (188) & $\begin{array}{l}\text { P. flavidorsum } \\
\text { SHK1-27 }\end{array}$ & Marine sediment & Anthraquinone & K562 & $12.6 \mu \mathrm{M}$ & $\begin{array}{l}\text { Induce cell cycle } \\
\text { arrest at } \mathrm{G}_{2} / \mathrm{M} \\
\text { transition }\end{array}$ & [126] \\
\hline Averantin (189) & $\begin{array}{l}\text { P. flavidorsum } \\
\text { SHK1-27 }\end{array}$ & Marine sediment & Anthraquinone & K562 & $12.6 \mu \mathrm{M}$ & & [126] \\
\hline 190 & P. terrestre & Marine sediment & Polyketide & A549, P388 & $(5.3,15.7) \mu \mathrm{M}$ & & [128] \\
\hline
\end{tabular}


Table A1. Cont.

\begin{tabular}{|c|c|c|c|c|c|c|c|}
\hline Metabolites & Producing Stain & $\begin{array}{l}\text { Environment } \\
\text { Source }\end{array}$ & Type & $\begin{array}{l}\text { Cell Lines/Brine } \\
\text { Shrimp }\end{array}$ & $\mathrm{IC}_{50}, \mathrm{LD}_{50}$, or IR (\%) & Target & References \\
\hline 191 & P. terrestre & Marine sediment & Polyketide & A549, P388 & $(7.6,10.5) \mu \mathrm{M}$ & & {$[128]$} \\
\hline Dihydrobisvertinolone (192) & P. terrestre & Marine sediment & Polyketide & A549, P388 & $(0.52,1.7) \mu \mathrm{M}$ & & [128] \\
\hline 193 & P. terrestre & Marine sediment & Polyketide & A549 & $1.4 \mu \mathrm{M}$ & & {$[128]$} \\
\hline 194 & P. terrestre & Marine sediment & Polyketide & A549, P388 & $(2.1,2.8) \mu \mathrm{M}$ & & [129] \\
\hline 195 & P. terrestre & Marine sediment & Polyketide & A549, P388 & $(4.3,8.8) \mu \mathrm{M}$ & & [129] \\
\hline Trichodimerol (196) & P. terrestre & Marine sediment & Polyketide & A549, P388 & $(4.7,0.33) \mu \mathrm{M}$ & & [129] \\
\hline Chloctanspirone A (197) & P. terrestre & Marine sediment & Polyketide & HL-60, A549 & $(9.2,39.7) \mu \mathrm{M}$ & & [131] \\
\hline Chloctanspirone B (198) & P. terrestre & Marine sediment & Polyketide & HL-60 & $37.8 \mu \mathrm{M}$ & & [131] \\
\hline $\begin{array}{l}(10 E, 15 S)-10,11-D e h y d r o c u r v u l a r i n \\
(199)\end{array}$ & $\begin{array}{l}\text { Penicillium } \mathrm{sp} . \\
\text { DRF2 }\end{array}$ & Marine sponge & Macrolide & 36 tumor cell lines & $0.28 \sim 6 \mu \mathrm{M}$ & & {$[133,134]$} \\
\hline Curvularin (200) & $\begin{array}{l}\text { Penicillium sp. } \\
\text { DRF2 }\end{array}$ & Marine sponge & Macrolide & & & HSP90 inhibitor & [132] \\
\hline Tanzawaic acid $P(201)$ & $\begin{array}{l}\text { Penicillium sp. } \\
\quad \text { CF07370 }\end{array}$ & Marine sediment & Polyketide & Jurkat, K562, Raji & $(28.6,30.2,20.3) \mu \mathrm{M}$ & $\begin{array}{c}\text { Active the } \\
\text { mitochondrial } \\
\text { apoptotic pathway }\end{array}$ & [137] \\
\hline Tanzawaic acid D (202) & P. steckii & Marine (not clear) & Polyketide & & & $\begin{array}{l}\text { Bind to the FOXO1 } \\
\text { which regulates } \\
\text { EFGR signaling and } \\
\text { stabilizes the } \\
\text { FOXO1-DNA } \\
\text { conformation }\end{array}$ & [138] \\
\hline Penicimutamide A (203) & $\begin{array}{c}\text { Mutant } P . \\
\text { purpurogenum } \\
\text { G59 }\end{array}$ & Marine soil & Lipopepetide & $\begin{array}{l}\text { K562, HL-60, Hela, } \\
\text { BGC-823, MCF-7 }\end{array}$ & $\begin{array}{c}\mathrm{IR} \%(100 \mu \mathrm{g} / \mathrm{mL})= \\
10 \sim 40 \%\end{array}$ & & [140] \\
\hline Penicimutamide B (204) & $\begin{array}{c}\text { Mutant } P . \\
\text { purpurogenum } \\
\text { G59 }\end{array}$ & Marine soil & Lipopepetide & $\begin{array}{l}\text { K562, HL-60, Hela, } \\
\text { BGC-823, MCF-7 }\end{array}$ & $\begin{array}{c}\mathrm{IR} \%(100 \mu \mathrm{g} / \mathrm{mL})= \\
25 \sim 40 \%\end{array}$ & & [140] \\
\hline Penicimutamide C (205) & $\begin{array}{c}\text { Mutant } P . \\
\text { purpurogenum } \\
\text { G59 }\end{array}$ & Marine soil & Lipopepetide & $\begin{array}{l}\text { K562, HL-60, Hela, } \\
\text { BGC-823, MCF-7 }\end{array}$ & $\begin{array}{c}\mathrm{IR} \%(100 \mu \mathrm{g} / \mathrm{mL})= \\
10 \sim 40 \%\end{array}$ & & [140] \\
\hline Penicimutamide D (206) & $\begin{array}{c}\text { Mutant } P . \\
\text { purpurogenum } \\
\text { G59 } \\
\end{array}$ & Marine soil & Lipopepetide & $\begin{array}{l}\text { K562, HL-60, Hela, } \\
\text { BGC-823, MCF-7 }\end{array}$ & $\begin{array}{c}\mathrm{IR} \%(100 \mu \mathrm{g} / \mathrm{mL})= \\
20 \sim 40 \%\end{array}$ & & [140] \\
\hline
\end{tabular}


Table A1. Cont.

\begin{tabular}{|c|c|c|c|c|c|c|c|}
\hline Metabolites & Producing Stain & $\begin{array}{l}\text { Environment } \\
\text { Source }\end{array}$ & Type & $\begin{array}{l}\text { Cell Lines/Brine } \\
\text { Shrimp }\end{array}$ & $\mathrm{IC}_{50}, \mathrm{LD}_{50}$, or IR (\%) & Target & References \\
\hline Penicimutamide E (207) & $\begin{array}{c}\text { Mutant } P . \\
\text { purpurogenum } \\
\text { G59 }\end{array}$ & Marine soil & Lipopepetide & $\begin{array}{l}\text { K562, HL-60, Hela, } \\
\text { BGC-823, MCF-7 }\end{array}$ & $\begin{array}{c}\mathrm{IR} \%(100 \mu \mathrm{g} / \mathrm{mL})= \\
10 \sim 45 \%\end{array}$ & & [140] \\
\hline Penicimutamide F (208) & $\begin{array}{c}\text { Mutant } P \text {. } \\
\text { purpurogenum } \\
\text { G59 }\end{array}$ & Marine soil & Lipopepetide & $\begin{array}{l}\text { K562, HL-60, Hela, } \\
\text { BGC-823, MCF-7 }\end{array}$ & $\begin{array}{c}\mathrm{IR} \%(100 \mu \mathrm{g} / \mathrm{mL})= \\
10 \sim 50 \%\end{array}$ & & [140] \\
\hline Penicimutamide G (209) & $\begin{array}{c}\text { Mutant } P . \\
\text { purpurogenum } \\
\text { G59 }\end{array}$ & Marine soil & Lipopepetide & $\begin{array}{l}\text { K562, HL-60, Hela, } \\
\text { BGC-823, MCF-7 }\end{array}$ & $\begin{array}{c}\mathrm{IR} \%(100 \mu \mathrm{g} / \mathrm{mL})= \\
10 \sim 20 \%\end{array}$ & & [140] \\
\hline Fellutamide A (210) & P. fellutanum & Marine fish & Lipopepetide & P388, L1210 & $(0.2,0.8) \mu \mathrm{g} / \mathrm{mL}$ & & [139] \\
\hline Fellutamide B (211) & P. fellutanum & Marine fish & Lipopepetide & P388, L1210 & $(0.1,0.7) \mu \mathrm{g} / \mathrm{mL}$ & & [139] \\
\hline Fellutamide C (212) & $\begin{array}{c}\text { Mutant } P . \\
\text { purpurogenum } \\
\text { G59 }\end{array}$ & Marine soil & Lipopepetide & $\begin{array}{l}\text { K562, HL-60, Hela, } \\
\text { BGC-823, MCF-7 }\end{array}$ & $\begin{array}{c}\mathrm{IR} \%(100 \mu \mathrm{g} / \mathrm{mL})= \\
30 \sim 50 \%\end{array}$ & & [140] \\
\hline Peniphenylane A (213) & $\begin{array}{l}\text { P. fellutanum } \\
\text { HDN14-323 }\end{array}$ & Deep sea sediment & Polyphenol & Hela & $14.5 \mu \mathrm{M}$ & & [141] \\
\hline Peniphenylane B (214) & $\begin{array}{l}\text { P. fellutanum } \\
\text { HDN14-323 }\end{array}$ & Deep sea sediment & Polyphenol & Hela, HCT-116 & $(11.4,15.8) \mu \mathrm{M}$ & & [141] \\
\hline Peniphenylane D (215) & $\begin{array}{l}\text { P. fellutanum } \\
\text { HDN14-323 }\end{array}$ & Deep sea sediment & Polyphenol & Hela, HL-60, HCT-116 & $(9.3,18.2,31.7) \mu \mathrm{M}$ & & [141] \\
\hline Peniphenylane F (216) & $\begin{array}{l}\text { P. fellutanum } \\
\text { HDN14-323 }\end{array}$ & Deep sea sediment & Polyphenol & Hela & $29.3 \mu \mathrm{M}$ & & [141] \\
\hline Peniphenylane G (217) & $\begin{array}{l}\text { P. fellutanum } \\
\text { HDN14-323 }\end{array}$ & Deep sea sediment & Polyphenol & Hela, HL-60, HCT-116 & $(16.6,23.2,24.7) \mu \mathrm{M}$ & & [141] \\
\hline Terrestol B (218) & P. terrestre & Marine sediment & Polyphenol & $\begin{array}{l}\text { HL-60, MOLT-4, A549, } \\
\text { BEL-7402 }\end{array}$ & $(6.1,5.8,18.3,62.3) \mu \mathrm{M}$ & & [142] \\
\hline Terrestol C (219) & P. terrestre & Marine sediment & Polyphenol & $\begin{array}{l}\text { HL-60, MOLT-4, A549, } \\
\text { BEL-7402 }\end{array}$ & $(5.5,5.6,18.2,57.3) \mu \mathrm{M}$ & & [142] \\
\hline Terrestol D (220) & P. terrestre & Marine sediment & Polyphenol & $\begin{array}{l}\text { HL-60, MOLT-4, A549, } \\
\text { BEL-7402 }\end{array}$ & $(5.3,5.5,14.3,38.5) \mu \mathrm{M}$ & & [142] \\
\hline Terrestol E (221) & P. terrestre & Marine sediment & Polyphenol & $\begin{array}{l}\text { HL-60, MOLT-4, A549, } \\
\text { BEL-7402 }\end{array}$ & $(54.7,6.4,9.6,59.0) \mu \mathrm{M}$ & & [142] \\
\hline
\end{tabular}


Table A1. Cont.

\begin{tabular}{|c|c|c|c|c|c|c|c|}
\hline Metabolites & Producing Stain & $\begin{array}{l}\text { Environment } \\
\text { Source }\end{array}$ & Type & $\begin{array}{l}\text { Cell Lines/Brine } \\
\text { Shrimp }\end{array}$ & $\mathrm{IC}_{50}, \mathrm{LD}_{50}$, or IR (\%) & Target & References \\
\hline Terrestol F (222) & P. terrestre & Marine sediment & Polyphenol & $\begin{array}{l}\text { HL-60, MOLT-4, A549, } \\
\text { BEL-7402 }\end{array}$ & $\begin{array}{c}(55.0,58.1,13.8 \\
63.2) \mu \mathrm{M}\end{array}$ & & [142] \\
\hline Terrestol G (223) & P. terrestre & Marine sediment & Polyphenol & $\begin{array}{l}\text { HL-60, MOLT-4, A549, } \\
\text { BEL-7402 }\end{array}$ & $(5.1,6.5,5.7,6.0) \mu \mathrm{M}$ & & [142] \\
\hline Terrestol H (224) & P. terrestre & Marine sediment & Polyphenol & $\begin{array}{l}\text { HL-60, MOLT-4, A549, } \\
\text { BEL-7402 }\end{array}$ & $(6.3,5.8,33.8,61.9) \mu \mathrm{M}$ & & {$[142]$} \\
\hline Terrestol A (225) & P. terrestre & Marine sediment & Polyphenol & $\begin{array}{l}\text { HL-60, MOLT-4, A549, } \\
\text { BEL-7402 }\end{array}$ & $(33.3,5.5,23.5,57.0) \mu \mathrm{M}$ & & [142] \\
\hline Expansol A (226) & $\begin{array}{l}\text { P. expansum } \\
091006\end{array}$ & Mangrove & Polyphenol & HL-60 & $15.7 \mu \mathrm{M}$ & & [143] \\
\hline Expansol B (227) & $\begin{array}{l}\text { P. expansum } \\
091006\end{array}$ & Mangrove & Polyphenol & HL-60, A549 & $(5.4,1.9) \mu \mathrm{M}$ & & {$[143,144]$} \\
\hline Expansol C (228) & $\begin{array}{l}\text { P. expansum } \\
091006\end{array}$ & Mangrove & Polyphenol & HL-60 & $18.2 \mu \mathrm{M}$ & & [143] \\
\hline Expansol E (229) & $\begin{array}{l}\text { P. expansum } \\
091006\end{array}$ & Mangrove & Polyphenol & HL-60 & $20.8 \mu \mathrm{M}$ & & [143] \\
\hline Patulin (230) & Penicillium sp. & Marine alga & Other & $\begin{array}{c}\text { P388, BSY-1, MCF-7, } \\
\text { HCC2998, NCI-H522, } \\
\text { DMS114, OVCAR-3, } \\
\text { MKN1 }\end{array}$ & $\begin{array}{c}(0.06,0.34,0.65,1.54 \\
0.30,0.57,0.37 \\
0.39) \mu \mathrm{g} / \mathrm{mL}\end{array}$ & $\begin{array}{l}\text { Potassium-uptake } \\
\text { inhibitor } \\
\text { Ion flux across cell } \\
\text { membranes inducer }\end{array}$ & [111] \\
\hline (+)-Epiepoxydon (231) & Penicillium sp. & Marine alga & Other & P388 & $0.2 \mu \mathrm{g} / \mathrm{mL}$ & & [111] \\
\hline 232 & Penicillium sp. & Mangrove & Other & KB, KBv200 & $(6,10) \mu \mathrm{g} / \mathrm{mL}$ & & [145] \\
\hline Penicillic acid (233) & Penicillium sp. & Sea water & Other & POS1, AT6-1, L299 & $(7.8,29.4,12.9) \mu \mathrm{M}$ & & [84] \\
\hline
\end{tabular}




\section{References}

1. Lindequist, U. Marine-Derived Pharmaceuticals-Challenges and Opportunities. Biomol. Ther. 2016, 24, 561-571. [CrossRef] [PubMed]

2. Wang, H.; Hong, J.; Yin, J.; Moon, H.R.; Liu, Y.; Wei, X.; Oh, D.-C.; Jung, J.H. Dimeric Octaketide Spiroketals from the Jellyfish-Derived Fungus Paecilomyces variotii J08NF-1. J. Nat. Prod. 2015, 78, 2832-2836. [CrossRef] [PubMed]

3. Lee, Y.M.; Kim, M.J.; Li, H.; Zhang, P.; Bao, B.; Lee, K.J.; Jung, J.H. Marine-derived Aspergillus species as a source of bioactive secondary metabolites. Mar. Biotechnol. 2013, 15, 499-519. [CrossRef] [PubMed]

4. Ding, G.; Song, Y.C.; Chen, J.R.; Xu, C.; Ge, H.M.; Wang, X.T.; Tan, R.X. Chaetoglobosin U, a cytochalasan alkaloid from endophytic Chaetomium globosum IFB-E019. J. Nat. Prod. 2006, 69, 302-304. [CrossRef] [PubMed]

5. Zhang, J.; Ge, H.M.; Jiao, R.H.; Li, J.; Peng, H.; Wang, Y.R.; Wu, J.H.; Song, Y.C.; Tan, R.X. Cytotoxic chaetoglobosins from the endophyte Chaetomium globosum. Planta Med. 2010, 76, 1910-1914. [CrossRef] [PubMed]

6. Huang, S.; Chen, H.; Li, W.; Zhu, X.; Ding, W.; Li, C. Bioactive Chaetoglobosins from the Mangrove Endophytic Fungus Penicillium chrysogenum. Mar. Drugs 2016, 14, 172. [CrossRef] [PubMed]

7. Iwamoto, C.; Yamada, T.; Ito, Y.; Minoura, K.; Numata, A. Cytotoxic cytochalasans from a Penicillium species separated from a marine alga. Tetrahedron 2001, 57, 2997-3004. [CrossRef]

8. Numata, A.; Takahashi, C.; Ito, Y.; Minoura, K.; Yamada, T.; Matsuda, C.; Nomoto, K. Penochalasins, a novel class of cytotoxic cytochalasans from a Penicillium species separated from a marine alga: Structure determination and solution conformation. J. Chem. Soc. Perkin 1 1996, 3, 239-245. [CrossRef]

9. Knudsen, P.B.; Hanna, B.; Ohl, S.; Sellner, L.; Zenz, T.; Dohner, H.; Stilgenbauer, S.; Larsen, T.O.; Lichter, P.; Seiffert, M. Chaetoglobosin A preferentially induces apoptosis in chronic lymphocytic leukemia cells by targeting the cytoskeleton. Leukemia 2014, 28, 1289-1298. [CrossRef] [PubMed]

10. Schümann, J.; Hertweck, C. Molecular basis of cytochalasan biosynthesis in fungi: Gene cluster analysis and evidence for the involvement of a PKS-NRPS hybrid synthase by RNA silencing. J. Am. Chem. Soc. 2007, 129, 9564-9565. [CrossRef] [PubMed]

11. McDougall, J. Antiviral action of gliotoxin. Arch. Virol. 1969, 27, 255-267. [CrossRef]

12. Sun, Y.; Takada, K.; Takemoto, Y.; Yoshida, M.; Nogi, Y.; Okada, S.; Matsunaga, S. Gliotoxin analogues from a marine-derived fungus, Penicillium sp., and their cytotoxic and histone methyltransferase inhibitory activities. J. Nat. Prod. 2011, 75, 111-114. [CrossRef] [PubMed]

13. Vigushin, D.; Mirsaidi, N.; Brooke, G.; Sun, C.; Pace, P.; Inman, L.; Moody, C.; Coombes, R. Gliotoxin is a dual inhibitor of farnesyltransferase and geranylgeranyltransferase I with antitumor activity against breast cancer in vivo. Med. Oncol. 2004, 21, 21-30. [CrossRef]

14. Meng, L.-H.; Wang, C.-Y.; Mándi, A.; Li, X.-M.; Hu, X.-Y.; Kassack, M.U.; Kurtán, T.; Wang, B.-G. Three Diketopiperazine Alkaloids with Spirocyclic Skeletons and One Bisthiodiketopiperazine Derivative from the Mangrove-Derived Endophytic Fungus Penicillium brocae MA-231. Org. Lett. 2016, 18, 5304-5307. [CrossRef] [PubMed]

15. Jiang, C.-S.; Guo, Y.-W. Epipolythiodioxopiperazines from fungi: Chemistry and bioactivities. Mini Rev. Med. Chem. 2011, 11, 728-745. [CrossRef] [PubMed]

16. Meng, L.-H.; Li, X.-M.; Lv, C.-T.; Huang, C.-G.; Wang, B.-G. Brocazines A-F, cytotoxic bisthiodiketopiperazine derivatives from Penicillium brocae MA-231, an endophytic fungus derived from the marine mangrove plant Avicennia marina. J. Nat. Prod. 2014, 77, 1921-1927. [CrossRef] [PubMed]

17. Liu, Y.; Li, X.-M.; Meng, L.-H.; Jiang, W.-L.; Xu, G.-M.; Huang, C.-G.; Wang, B.-G. Bisthiodiketopiperazines and acorane sesquiterpenes produced by the marine-derived fungus Penicillium adametzioides AS-53 on different culture media. J. Nat. Prod. 2015, 78, 1294-1299. [CrossRef] [PubMed]

18. Orfali, R.S.; Aly, A.H.; Ebrahim, W.; Abdel-Aziz, M.S.; Müller, W.E.; Lin, W.; Daletos, G.; Proksch, P. Pretrichodermamide $\mathrm{C}$ and $\mathrm{N}$-methylpretrichodermamide $\mathrm{B}$, two new cytotoxic epidithiodiketopiperazines from hyper saline lake derived Penicillium sp. Phytochem. Lett. 2015, 11, 168-172. [CrossRef]

19. Yurchenko, A.N.; Smetanina, O.F.; Ivanets, E.V.; Kalinovsky, A.I.; Khudyakova, Y.V.; Kirichuk, N.N.; Popov, R.S.; Bokemeyer, C.; Von Amsberg, G.; Chingizova, E.A. Pretrichodermamides D-F from a Marine Algicolous Fungus Penicillium sp. KMM 4672. Mar. Drugs 2016, 14, 122. [CrossRef] [PubMed] 
20. Aninat, C.; Andre, F.; Delaforge, M. Oxidative metabolism by P450 and function coupling to efflux systems: Modulation of mycotoxin toxicity. Food Addit. Contam. 2005, 22, 361-368. [CrossRef] [PubMed]

21. Du, L.; Feng, T.; Zhao, B.; Li, D.; Cai, S.; Zhu, T.; Wang, F.; Xiao, X.; Gu, Q. Alkaloids from a deep ocean sediment-derived fungus Penicillium sp. and their antitumor activities. J. Antibiot. 2010, 63, 165-170. [CrossRef] [PubMed]

22. Du, L.; Li, D.; Zhu, T.; Cai, S.; Wang, F.; Xiao, X.; Gu, Q. New alkaloids and diterpenes from a deep ocean sediment derived fungus Penicillium sp. Tetrahedron 2009, 65, 1033-1039. [CrossRef]

23. Shang, Z.; Li, X.; Meng, L.; Li, C.; Gao, S.; Huang, C.; Wang, B. Chemical profile of the secondary metabolites produced by a deep sea sediment-derived fungus Penicillium commune SD-118. Chin. J. Oceanol. Limnol. 2012, 30, 305-314. [CrossRef]

24. Fang, S.-M.; Wu, C.-J.; Li, C.-W.; Cui, C.-B. A practical strategy to discover new antitumor compounds by activating silent metabolite production in fungi by diethyl sulphate mutagenesis. Mar. Drugs 2014, 12, 1788-1814. [CrossRef] [PubMed]

25. Chai, Y.-J.; Cui, C.-B.; Li, C.-W.; Wu, C.-J.; Tian, C.-K.; Hua, W. Activation of the dormant secondary metabolite production by introducing gentamicin-resistance in a marine-derived Penicillium purpurogenum G59. Mar. Drugs 2012, 10, 559-582. [CrossRef] [PubMed]

26. Hauser, D.; Weber, H.; Sigg, H. Isolation and configuration of Chaetocin. Helv. Chim. Acta 1970, 53, $1061-1073$. [CrossRef] [PubMed]

27. Katagiri, K.; Sato, K.; Hayakawa, S.; Matsushima, T.; Minato, H. Verticillin A, a new anti-biotic from Verticillium sp. J. Antibiot. 1970, 23, 420-422. [CrossRef] [PubMed]

28. Son, B.; Jensen, P.; Kauffman, C.; Fenical, W. New Cytotoxic Epidithiodioxopiperazines Related to Verticillin A From A Marine Isolate of the Fungus Penicillium. Nat. Prod. Lett. 1999, 13, 213-222. [CrossRef]

29. Greiner, D.; Bonaldi, T.; Eskeland, R.; Roemer, E.; Imhof, A. Identification of a specific inhibitor of the histone methyltransferase SU (VAR) 3-9. Nat. Chem. Biol. 2005, 1, 143-145. [CrossRef] [PubMed]

30. Chen, Y.; Miao, Z.-H.; Zhao, W.-M.; Ding, J. The p53 pathway is synergized by p38 MAPK signaling to mediate 11,11'-dideoxyverticillin-induced G2/M arrest. FEBS Lett. 2005, 579, 3683-3690. [CrossRef] [PubMed]

31. Zhang, Y.-X.; Chen, Y.; Guo, X.-N.; Zhang, X.-W.; Zhao, W.-M.; Zhong, L.; Zhou, J.; Xi, Y.; Lin, L.-P.; Ding, J. 11,11'-dideoxy-verticillin: A natural compound possessing growth factor receptor tyrosine kinase-inhibitory effect with anti-tumor activity. Anti-Cancer Drugs 2005, 16, 515-524. [CrossRef] [PubMed]

32. Kim, J.; Ashenhurst, J.A.; Movassaghi, M. Total synthesis of (+)-11,11'-dideoxyverticillin A. Science 2009, 324, 238-241. [CrossRef] [PubMed]

33. Kim, J.; Movassaghi, M. Biogenetically-inspired total synthesis of epidithiodiketopiperazines and related alkaloids. Acc. Chem. Res. 2015, 48, 1159-1171. [CrossRef] [PubMed]

34. Sallam, A.A.; Houssen, W.E.; Gissendanner, C.R.; Orabi, K.Y.; Foudah, A.I.; El Sayed, K.A. Bioguided discovery and pharmacophore modeling of the mycotoxic indole diterpene alkaloids penitrems as breast cancer proliferation, migration, and invasion inhibitors. MedChem Comm 2013, 4, 1360-1369. [CrossRef] [PubMed]

35. Sings, H.; Singh, S. Tremorgenic and nontremorgenic 2,3-fused indole diterpenoids. Alkaloids Chem. Biol. 2003, 60, 51-163. [PubMed]

36. Saikia, S.; Nicholson, M.J.; Young, C.; Parker, E.J.; Scott, B. The genetic basis for indole-diterpene chemical diversity in filamentous fungi. Mycol. Res. 2008, 112, 184-199. [CrossRef] [PubMed]

37. Cavanagh, J.; Holton, J.; Nolan, C.; Ray, D.; Naik, J.; Mantle, P. The effects of the tremorgenic mycotoxin penitrem A on the rat cerebellum. Vet. Pathol. 1998, 35, 53-63. [CrossRef] [PubMed]

38. Numata, A.; Takahashi, C.; Ito, Y.; Takada, T.; Kawai, K.; Usami, Y.; Matsumura, E.; Imachi, M.; Ito, T.; Hasegawa, T. Communesins, cytotoxic metabolites of a fungus isolated from a marine alga. Tetrahedron Lett. 1993, 34, 2355-2358. [CrossRef]

39. Jadulco, R.; Edrada, R.A.; Ebel, R.; Berg, A.; Schaumann, K.; Wray, V.; Steube, K.; Proksch, P. New Communesin Derivatives from the Fungus Penicillium sp. Derived from the Mediterranean Sponge Axinella v errucosa. J. Nat. Prod. 2004, 67, 78-81. [CrossRef] [PubMed]

40. Vansteelandt, M.; Kerzaon, I.; Blanchet, E.; Tankoua, O.F.; Du Pont, T.R.; Joubert, Y.; Monteau, F.; Le Bizec, B.; Frisvad, J.C.; Pouchus, Y.F. Patulin and secondary metabolite production by marine-derived Penicillium strains. Fungal Biol. 2012, 116, 954-961. [CrossRef] [PubMed] 
41. Crawley, S.L.; Funk, R.L. A synthetic approach to nomofungin/communesin B. Org. Lett. 2003, 5, 3169-3171. [CrossRef] [PubMed]

42. Zhang, P.; Li, X.-M.; Liu, H.; Li, X.; Wang, B.-G. Two new alkaloids from Penicillium oxalicum EN-201, an endophytic fungus derived from the marine mangrove plant Rhizophora stylosa. Phytochem. Lett. 2015, 13, 160-164. [CrossRef]

43. An, C.-Y.; Li, X.-M.; Li, C.-S.; Xu, G.-M.; Wang, B.-G. Prenylated indolediketopiperazine peroxides and related homologues from the marine sediment-derived fungus Penicillium brefeldianum SD-273. Mar. Drugs 2014, 12, 746-756. [CrossRef] [PubMed]

44. Li, C.S.; Li, X.M.; An, C.Y.; Wang, B.G. Prenylated Indole Alkaloid Derivatives from Marine Sediment-Derived Fungus Penicillium paneum SD-44. Helv. Chim. Acta 2014, 97, 1440-1444. [CrossRef]

45. Asiri, I.A.; Badr, J.M.; Youssef, D.T. Penicillivinacine, antimigratory diketopiperazine alkaloid from the marine-derived fungus Penicillium vinaceum. Phytochem. Lett. 2015, 13, 53-58. [CrossRef]

46. Shaala, L.A.; Youssef, D.T. Identification and bioactivity of compounds from the fungus Penicillium sp. CYE-87 isolated from a marine tunicate. Mar. Drugs 2015, 13, 1698-1709. [CrossRef] [PubMed]

47. Chen, L.; Huang, K.; Zhong, P. Tumonoic Acids K and L, Novel Metabolites from the Marine-Derived Fungus Penicillium citrinum. Heterocycles 2012, 85, 413-419. [CrossRef]

48. Chen, L.; Zhou, T.; Zhao, Y.-Y. Four new penicitrinols and two new penicillenols from the marine-derived fungus Penicillium citrinum. Heterocycles 2015, 91, 1007-1016. [CrossRef]

49. Lin, Z.-J.; Lu, Z.-Y.; Zhu, T.-J.; Fang, Y.-C.; Gu, Q.-Q.; Zhu, W.-M. Penicillenols from Penicillium sp. GQ-7, an endophytic fungus associated with Aegiceras corniculatum. Chem. Pharm. Bull. 2008, 56, 217-221. [CrossRef] [PubMed]

50. Liu, Q.-Y.; Zhou, T.; Zhao, Y.-Y.; Chen, L.; Gong, M.-W.; Xia, Q.-W.; Ying, M.-G.; Zheng, Q.-H.; Zhang, Q.-Q. Antitumor effects and related mechanisms of penicitrinine A, a novel alkaloid with a unique spiro skeleton from the marine fungus Penicillium citrinum. Mar. Drugs 2015, 13, 4733-4753. [CrossRef] [PubMed]

51. Jafari, E.; Khajouei, M.R.; Hassanzadeh, F.; Hakimelahi, G.H.; Khodarahmi, G.A. Quinazolinone and quinazoline derivatives: Recent structures with potent antimicrobial and cytotoxic activities. Res. Pharm. Sci. 2016, 11, 1-14. [PubMed]

52. He, J.; Lion, U.; Sattler, I.; Gollmick, F.A.; Grabley, S.; Cai, J.; Meiners, M.; Schünke, H.; Schaumann, K.; Dechert, U. Diastereomeric Quinolinone Alkaloids from the Marine-Derived Fungus Penicillium j anczewskii. J. Nat. Prod. 2005, 68, 1397-1399. [CrossRef] [PubMed]

53. Gao, H.; Zhang, L.; Zhu, T.; Gu, Q.; Li, D. Unusual pyrrolyl 4-quinolinone alkaloids from the marine-derived fungus Penicillium sp. ghq208. Chem. Pharm. Bull. 2012, 60, 1458-1460. [CrossRef] [PubMed]

54. Shao, C.-L.; Wang, C.-Y.; Gu, Y.-C.; Wei, M.-Y.; Pan, J.-H.; Deng, D.-S.; She, Z.-G.; Lin, Y.-C. Penicinoline, a new pyrrolyl 4-quinolinone alkaloid with an unprecedented ring system from an endophytic fungus Penicillium sp. Bioorg. Med. Chem. Lett. 2010, 20, 3284-3286. [CrossRef] [PubMed]

55. Shen, S.; Li, W.; Wang, J. A novel and other bioactive secondary metabolites from a marine fungus Penicillium oxalicum 0312f1. Nat. Prod. Rep. 2013, 27, 2286-2291. [CrossRef] [PubMed]

56. An, C.Y.; Li, X.M.; Li, C.S.; Gao, S.S.; Shang, Z.; Wang, B.G. Triazoles and Other N-Containing Metabolites from the Marine-Derived Endophytic Fungus Penicillium chrysogenum EN-118. Helv. Chim. Acta 2013, 96, 682-687. [CrossRef]

57. Li, C.-S.; Li, X.-M.; Gao, S.-S.; Lu, Y.-H.; Wang, B.-G. Cytotoxic anthranilic acid derivatives from deep sea sediment-derived fungus Penicillium paneum SD-44. Mar. Drugs 2013, 11, 3068-3076. [CrossRef] [PubMed]

58. Li, C.-S.; An, C.-Y.; Li, X.-M.; Gao, S.-S.; Cui, C.-M.; Sun, H.-F.; Wang, B.-G. Triazole and dihydroimidazole alkaloids from the marine sediment-derived fungus Penicillium paneum SD-44. J. Nat. Prod. 2011, 74, 1331-1334. [CrossRef] [PubMed]

59. Li, J.; Yang, X.; Lin, Y.; Yuan, J.; Lu, Y.; Zhu, X.; Li, J.; Li, M.; Lin, Y.; He, J. Meroterpenes and azaphilones from marine mangrove endophytic fungus Penicillium 303. Fitoterapia 2014, 97, 241-246. [CrossRef] [PubMed]

60. Bringmann, G.; Lang, G.; Gulder, T.A.; Tsuruta, H.; Mühlbacher, J.; Maksimenka, K.; Steffens, S.; Schaumann, K.; Stöhr, R.; Wiese, J. The first sorbicillinoid alkaloids, the antileukemic sorbicillactones A and B, from a sponge-derived Penicillium chrysogenum strain. Tetrahedron 2005, 61, 7252-7265. [CrossRef]

61. Bringmann, G.; Gulder, T.A.; Lang, G.; Schmitt, S.; Stöhr, R.; Wiese, J.; Nagel, K.; Imhoff, J.F. Large-scale biotechnological production of the antileukemic marine natural product sorbicillactone A. Mar. Drugs 2007, 5, 23-30. [CrossRef] [PubMed] 
62. Zhang, P.; Meng, L.H.; Mándi, A.; Kurtán, T.; Li, X.M.; Liu, Y.; Li, X.; Li, C.S.; Wang, B.G. Brocaeloids A-C, 4-Oxoquinoline and Indole Alkaloids with C-2 Reversed Prenylation from the Mangrove-Derived Endophytic Fungus Penicillium brocae. Eur. J. Org. Chem. 2014, 2014, 4029-4036. [CrossRef]

63. He, X.; Zhang, Z.; Chen, Y.; Che, Q.; Zhu, T.; Gu, Q.; Li, D. Varitatin A, a Highly Modified Fatty Acid Amide from Penicillium variabile Cultured with a DNA Methyltransferase Inhibitor. J. Nat. Prod. 2015, 78, 2841-2845. [CrossRef] [PubMed]

64. Zhang, Y.; Li, C.; Swenson, D.C.; Gloer, J.B.; Wicklow, D.T.; Dowd, P.F. Novel antiinsectan oxalicine alkaloids from two undescribed fungicolous Penicillium spp. Org. Lett. 2003, 5, 773-776. [CrossRef] [PubMed]

65. Takatsuki, A.; Suzuki, S.; Ando, K.; Tamura, G.; Arima, K. New antiviral antibiotics; xanthocillin X mono-and dimethylether, and methoxy-xanthocillin X dimethylether. I: Isolation and characterization. J. Antibiot. 1968, 21, 671-675. [CrossRef] [PubMed]

66. Rothe, W. Vorläufige Mitteilung über eine neues Antibiotikum. Pharmazie 1950, 5, 190.

67. Zhao, Y.; Chen, H.; Shang, Z.; Jiao, B.; Yuan, B.; Sun, W.; Wang, B.; Miao, M.; Huang, C. SD118-xanthocillin $\mathrm{X}(1)$, a novel marine agent extracted from Penicillium commune, induces autophagy through the inhibition of the MEK/ERK pathway. Mar. Drugs 2012, 10, 1345-1359. [CrossRef] [PubMed]

68. Sugawara, F.; Hallock, Y.; Bunkers, G.; Kenfield, D.; Strobel, G.; Yoshida, S. Phytoactive eremophilanes produced by the weed pathogen Drechslera gigantea. Biosci. Biotechnol. Biochem. 1993, 57, 236-239. [CrossRef] [PubMed]

69. Tirilly, Y.; Kloosterman, J.; Sipma, G.; Kettenes-Van den Bosch, J.J. A fungitoxic sesquiterpene from Hansfordia pulvinata. Phytochemistry 1983, 22, 2082-2083. [CrossRef]

70. Lin, A.; Wu, G.; Gu, Q.; Zhu, T.; Li, D. New eremophilane-type sesquiterpenes from an Antarctic deep sea derived fungus, Penicillium sp. PR19 N-1. Arch. Pharm. Res. 2014, 37, 839-844. [CrossRef] [PubMed]

71. Wu, G.; Lin, A.; Gu, Q.; Zhu, T.; Li, D. Four new chloro-eremophilane sesquiterpenes from an Antarctic deep sea derived fungus, Penicillium sp. PR19N-1. Mar. Drugs 2013, 11, 1399-1408. [CrossRef] [PubMed]

72. Huang, Y.F.; Qiao, L.; Lv, A.L.; Pei, Y.H.; Tian, L. Eremophilane sesquiterenes from the marine fungus Penicillium sp. BL27-2. Chin. Chem. Lett. 2008, 19, 562-564. [CrossRef]

73. Li, Y.; Ye, D.; Shao, Z.; Cui, C.; Che, Y. A sterol and spiroditerpenoids from a Penicillium sp. isolated from a deep sea sediment sample. Mar. Drugs 2012, 10, 497-508. [CrossRef] [PubMed]

74. Gao, S.-S.; Li, X.-M.; Li, C.-S.; Proksch, P.; Wang, B.-G. Penicisteroids A and B, antifungal and cytotoxic polyoxygenated steroids from the marine alga-derived endophytic fungus Penicillium chrysogenum QEN-24S. Bioorg. Med. Chem. Lett. 2011, 21, 2894-2897. [CrossRef] [PubMed]

75. Sun, Y.; Tian, L.; Huang, J.; Li, W.; Pei, Y.-H. Cytotoxic sterols from marine-derived fungus Pennicillium sp. Nat. Prod. Rep. 2006, 20, 381-384. [CrossRef] [PubMed]

76. Xin, Z.-H.; Zhu, T.-J.; Wang, W.-L.; Du, L.; Fang, Y.-C.; Gu, Q.-Q.; Zhu, W.-M. Isocoumarin derivatives from the sea squirt-derived fungus Penicillium stoloniferum QY2-10 and the halotolerant fungus Penicillium notatum B-52. Arch. Pharm. Res. 2007, 30, 816-819. [CrossRef] [PubMed]

77. Qi, J.; Shao, C.-L.; Liu, M.; Qi, X.; Wang, C.-Y. Bioactive steroids from a marine-derived fungus Penicillium sp. from the South China Sea. Chem. Nat. Compd. 2014, 3, 568-570. [CrossRef]

78. Menna, M.; Imperatore, C.; D'Aniello, F.; Aiello, A. Meroterpenes from marine invertebrates: Structures, occurrence, and ecological implications. Mar. Drugs 2013, 11, 1602-1643. [CrossRef] [PubMed]

79. Li, X.; Choi, H.D.; Kang, J.S.; Lee, C.-O.; Son, B.W. New polyoxygenated farnesylcyclohexenones, deacetoxyyanuthone A and its hydro derivative from the marine-derived fungus Penicillium sp. J. Nat. Prod. 2003, 66, 1499-1500. [CrossRef] [PubMed]

80. Zhuang, P.; Tang, X.-X.; Yi, Z.-W.; Qiu, Y.-K.; Wu, Z. Two new compounds from marine-derived fungus Penicillium sp. F11. J. Asian Nat. Prod. Res. 2012, 14, 197-203. [CrossRef] [PubMed]

81. Ding, Z.; Zhang, L.; Fu, J.; Che, Q.; Li, D.; Gu, Q.; Zhu, T. Phenylpyropenes E and F: New meroterpenes from the marine-derived fungus Penicillium concentricum ZLQ-69. J. Antibiot. 2015, 68, 748-751. [CrossRef] [PubMed]

82. Liao, L.; Lee, J.-H.; You, M.; Choi, T.J.; Park, W.; Lee, S.K.; Oh, D.-C.; Oh, K.-B.; Shin, J. Penicillipyrones A and B, meroterpenoids from a marine-derived Penicillium sp. fungus. J. Nat. Prod. 2014, 77, 406-410. [CrossRef] [PubMed]

83. Hanson, F.; Eble, T. An antiphage agent isolated from Aspergillus sp. J. Bacteriol. 1949, 58, 527-529. [PubMed] 
84. Vansteelandt, M.; Blanchet, E.; Egorov, M.; Petit, F.; Toupet, L.; Bondon, A.; Monteau, F.; Le Bizec, B.; Thomas, O.P.; Pouchus, Y.F. Ligerin, an antiproliferative chlorinated sesquiterpenoid from a marine-derived Penicillium strain. J. Nat. Prod. 2013, 76, 297-301. [CrossRef] [PubMed]

85. Blanchet, E.; Vansteelandt, M.; Bot, R.L.; Egorov, M.; Guitton, Y.; Pouchus, Y.F.; Grovel, O. Synthesis and antiproliferative activity of ligerin and new fumagillin analogs against osteosarcoma. Eur. J. Med. Chem. 2014, 79, 244-250. [CrossRef] [PubMed]

86. Keri, R.S.; Budagumpi, S.; Pai, R.K.; Balakrishna, R.G. Chromones as a privileged scaffold in drug discovery: A review. Eur. J. Med. Chem. 2014, 78, 340-374. [CrossRef] [PubMed]

87. Bao, J.; Luo, J.-F.; Qin, X.-C.; Xu, X.-Y.; Zhang, X.-Y.; Tu, Z.-C.; Qi, S.-H. Dihydrothiophene-condensed chromones from a marine-derived fungus Penicillium oxalicum and their structure-bioactivity relationship. Bioorg. Med. Chem. Lett. 2014, 24, 2433-2436. [CrossRef] [PubMed]

88. Sun, Y.-L.; Bao, J.; Liu, K.-S.; Zhang, X.-Y.; He, F.; Wang, Y.-F.; Nong, X.-H.; Qi, S.-H. Cytotoxic dihydrothiophene-condensed chromones from the marine-derived fungus Penicillium oxalicum. Planta Med. 2013, 79, 1474-1479. [CrossRef] [PubMed]

89. Wang, J.; Wang, Q.-L.; Nong, X.-H.; Zhang, X.-Y.; Xu, X.-Y.; Qi, S.-H.; Wang, Y.-F. Oxalicumone A, a new dihydrothiophene-condensed sulfur chromone induces apoptosis in leukemia cells through endoplasmic reticulum stress pathway. Eur. J. Pharmacol. 2016, 783, 47-55. [CrossRef] [PubMed]

90. Shi, S.; Guo, K.; Wang, X.; Chen, H.; Min, J.; Qi, S.; Zhao, W.; Li, W. Toxicity study of oxalicumone A, derived from a marine-derived fungus Penicillium oxalicum, in cultured renal epithelial cells. Mol. Med. Rep. 2017, 15, 2611-2619. [CrossRef] [PubMed]

91. Yi, L.; Cui, C.-B.; Li, C.-W.; Peng, J.-X.; Gu, Q.-Q. Chromosulfine, a novel cyclopentachromone sulfide produced by a marine-derived fungus after introduction of neomycin resistance. RSC Adv. 2016, 6, 43975-43979. [CrossRef]

92. Li, N.; Yi, Z.; Wang, Y.; Zhang, Q.; Zhong, T.; Qiu, Y.; Wu, Z.; Tang, X. Differential proteomic analysis of HL60 cells treated with secalonic acid F reveals caspase 3-induced cleavage of Rho GDP dissociation inhibitor 2. Oncol. Rep. 2012, 28, 2016-2022. [CrossRef] [PubMed]

93. Gao, X.; Sun, H.; Liu, D.; Zhang, J.; Zhang, J.; Yan, M.; Pan, X. Secalonic acid-F inhibited cell growth more effectively than 5-fluorouracil on hepatocellular carcinoma in vitro and in vivo. Neoplasma 2017, 64, 344-350. [CrossRef] [PubMed]

94. Hou, X.-M.; Wang, C.-Y.; Gu, Y.-C.; Shao, C.-L. Penimethavone A, a flavone from a gorgonian-derived fungus Penicillium chrysogenum. Nat. Prod. Rep. 2016, 30, 2274-2277. [CrossRef] [PubMed]

95. Chen, S.; Wang, J.; Wang, Z.; Lin, X.; Zhao, B.; Kaliaperumal, K.; Liao, X.; Tu, Z.; Li, J.; Xu, S. Structurally diverse secondary metabolites from a deep sea-derived fungus Penicillium chrysogenum SCSIO 41001 and their biological evaluation. Fitoterapia 2017, 117, 71-78. [CrossRef] [PubMed]

96. Chen, H.; Aktas, N.; Konuklugil, B.; Mandi, A.; Daletos, G.; Lin, W.; Dai, H.; Kurtan, T.; Proksch, P. A new fusarielin analogue from Penicillium sp. isolated from the Mediterranean sponge Ircinia oros. Tetrahedron Lett. 2015, 56, 5317-5320. [CrossRef]

97. Huang, Z.; Yang, J.; Cai, X.; She, Z.; Lin, Y. A new furanocoumarin from the mangrove endophytic fungus Penicillium sp.(ZH16). Nat. Prod. Rep. 2012, 26, 1291-1295. [CrossRef] [PubMed]

98. Hetherington, A.C.; Raistrick, H. On the production and chemical constitution of a new yellow colouring matter, citrinin, produced from glucose by Penicillium citrinum Thom. Philos. Trans. R. Soc. Lond. 1931, 220, 269-295. [CrossRef]

99. Chagas, G.M.; Campello, A.P.; Klüppel, M.; Lúcia, W. Mechanism of citrinin-induced dysfunction of mitochondria. I. Effects on respiration, enzyme activities and membrane potential of renal cortical mitochondria. J. Appl. Toxicol. 1992, 12, 123-129. [CrossRef] [PubMed]

100. He, Y.; Cox, R.J. The molecular steps of citrinin biosynthesis in fungi. Chem. Sci. 2016, 7, $2119-2127$. [CrossRef]

101. Subramani, R.; Kumar, R.; Prasad, P.; Aalbersberg, W. Cytotoxic and antibacterial substances against multi-drug resistant pathogens from marine sponge symbiont: Citrinin, a secondary metabolite of Penicillium sp. Asian Pac. J. Trop. Biomed. 2013, 3, 291-296. [CrossRef]

102. Stierle, A.A.; Stierle, D.B.; Kelly, K. Berkelic acid, a novel spiroketal with selective anticancer activity from an acid mine waste fungal extremophile. J. Org. Chem. 2006, 71, 5357-5360. [CrossRef] [PubMed] 
103. Wu, X.; Zhou, J.; Snider, B.B. Synthesis of (-)-Berkelic Acid. Angew. Chem. Int. Ed. 2009, 48, 1283-1286. [CrossRef] [PubMed]

104. Zhuravleva, O.I.; Sobolevskaya, M.P.; Afiyatullov, S.S.; Kirichuk, N.N.; Denisenko, V.A.; Dmitrenok, P.S.; Yurchenko, E.A.; Dyshlovoy, S.A. Sargassopenillines A-G, 6,6-spiroketals from the alga-derived fungi Penicillium thomii and Penicillium lividum. Mar. Drugs 2014, 12, 5930-5943. [CrossRef] [PubMed]

105. Perpelescu, M.; Kobayashi, J.I.; Furuta, M.; Ito, Y.; Izuta, S.; Takemura, M.; Suzuki, M.; Yoshida, S. Novel phenalenone derivatives from a marine-derived fungus exhibit distinct inhibition spectra against eukaryotic DNA polymerases. Biochemistry 2002, 41, 7610-7616. [CrossRef] [PubMed]

106. Chen, L.; Gong, M.-W.; Peng, Z.-F.; Zhou, T.; Ying, M.-G.; Zheng, Q.-H.; Liu, Q.-Y.; Zhang, Q.-Q. The marine fungal metabolite, dicitrinone $\mathrm{B}$, induces A375 cell apoptosis through the ROS-related caspase pathway. Mar. Drugs 2014, 12, 1939-1958. [CrossRef] [PubMed]

107. Sasaki, M.; Tsuda, M.; Sekiguchi, M.; Mikami, Y.; Kobayashi, J.I. Perinadine A, a Novel Tetracyclic Alkaloid from Marine-Derived Fungus Penicillium citrinum. Org. Lett. 2005, 7, 4261-4264. [CrossRef] [PubMed]

108. Julianti, E.; Lee, J.-H.; Liao, L.; Park, W.; Park, S.; Oh, D.-C.; Oh, K.-B.; Shin, J. New polyaromatic metabolites from a marine-derived fungus Penicillium sp. Org. Lett. 2013, 15, 1286-1289. [CrossRef] [PubMed]

109. Gao, S.-S.; Li, X.-M.; Zhang, Y.; Li, C.-S.; Cui, C.-M.; Wang, B.-G. Comazaphilones A-F, azaphilone derivatives from the marine sediment-derived fungus Penicillium commune QSD-17. J. Nat. Prod. 2011, 74, 256-261. [CrossRef] [PubMed]

110. Myobatake, Y.; Takeuchi, T.; Kuramochi, K.; Kuriyama, I.; Ishido, T.; Hirano, K.; Sugawara, F.; Yoshida, H.; Mizushina, Y. Pinophilins A and B, inhibitors of mammalian A-, B-, and Y-family DNA polymerases and human cancer cell proliferation. J. Nat. Prod. 2012, 75, 135-141. [CrossRef] [PubMed]

111. Iwamoto, C.; Minoura, K.; Oka, T.; Ohta, T.; Hagishita, S.; Numata, A. Absolute stereostructures of novel cytotoxic metabolites, penostatins A-E, from a Penicillium species separated from an Enteromorpha alga. Tetrahedron 1999, 55, 14353-14368. [CrossRef]

112. Iwamoto, C.; Minoura, K.; Hagishita, S.; Nomoto, K.; Numata, A. Penostatins F-I, novel cytotoxic metabolites from a Penicillium species separated from an Enteromorpha marine alga. J. Chem. Soc. Perkin 1 1998, 3, 449-456. [CrossRef]

113. Chen, Y.-P.; Yang, C.-G.; Wei, P.-Y.; Li, L.; Luo, D.-Q.; Zheng, Z.-H.; Lu, X.-H. Penostatin derivatives, a novel kind of protein phosphatase $1 \mathrm{~B}$ inhibitors isolated from solid cultures of the entomogenous fungus Isaria tenuipes. Molecules 2014, 19, 1663-1671. [CrossRef] [PubMed]

114. Snider, B.B.; Liu, T. Total Synthesis of ( \pm )-Deoxypenostatin A. Approaches to the Syntheses of Penostatins A and B. J. Org. Chem. 2000, 65, 8490-8498. [CrossRef] [PubMed]

115. Barriault, L.; Ang, P.J.; Lavigne, R.M. Rapid Assembly of the Bicyclo [5.3.1] undecenone Core of Penostatin F: A Successive Diels-Alder/Claisen Reaction Strategy with an Efficient Stereochemical Relay. Org. Lett. 2004, 6, 1317-1319. [CrossRef] [PubMed]

116. Crawford, J.M.; Townsend, C.A. New insights into the formation of fungal aromatic polyketides. Nat. Rev. Microbiol. 2010, 8, 879-889. [CrossRef] [PubMed]

117. Liu, B.; Wang, H.-F.; Zhang, L.-H.; Liu, F.; He, F.-J.; Bai, J.; Hua, H.-M.; Chen, G.; Pei, Y.-H. New compound with DNA Topo I inhibitory activity purified from Penicillium oxalicum HSY05. Nat. Prod. Rep. 2015, 29, 2197-2202. [CrossRef] [PubMed]

118. Wang, Y.; Wang, L.; Zhuang, Y.; Kong, F.; Zhang, C.; Zhu, W. Phenolic polyketides from the co-cultivation of marine-derived Penicillium sp. WC-29-5 and Streptomyces fradiae 007. Mar. Drugs 2014, 12, 2079-2088. [CrossRef] [PubMed]

119. Roullier, C.; Guitton, Y.; Valery, M.; Amand, S.; Prado, S.; Robiou du Pont, T.; Grovel, O.; Pouchus, Y.F. Automated detection of natural halogenated compounds from LC-MS profiles-Application to the isolation of bioactive chlorinated compounds from marine-derived fungi. Anal. Chem. 2016, 88, 9143-9150. [CrossRef] [PubMed]

120. Luo, H.; Li, X.-M.; Li, C.-S.; Wang, B.-G. Diphenyl ether and benzophenone derivatives from the marine mangrove-derived fungus Penicillium sp. MA-37. Phytochem. Lett. 2014, 9, 22-25. [CrossRef]

121. Nishida, H.; Tomoda, H.; Cao, J.; Araki, S.; Okuda, S.; Omura, S. Purpactins, new inhibitors of Acyl-CoA: Cholesterol acyltransferase produced by Penicillium purpurogenum. J. Antibiot. 1991, 44, 152-159. [CrossRef] [PubMed] 
122. Chung, M.-C.; Lee, H.; Chun, H.; Kho, Y. Penicillide, a nonpeptide calpain inhibitor, produced by Penicillium sp. J. Microbiol. Biotechnol. 1998, 8, 188-190.

123. Salituro, G.M.; Pettibone, D.J.; Clineschmidt, B.V.; Williamson, J.M.; Zink, D.L. Potent, non-peptidic oxytocin receptor antagonists from a natural source. Bioorg. Med. Chem. Lett. 1993, 3, 337-340. [CrossRef]

124. Gao, H.; Zhou, L.; Li, D.; Gu, Q.; Zhu, T.J. New Cytotoxic Metabolites from the Marine-Derived Fungus Penicillium sp. ZLN29. Helv. Chim. Acta 2013, 96, 514-519. [CrossRef]

125. Zhao, D.-L.; Shao, C.-L.; Zhang, Q.; Wang, K.-L.; Guan, F.-F.; Shi, T.; Wang, C.-Y. Azaphilone and diphenyl ether derivatives from a gorgonian-derived strain of the fungus Penicillium pinophilum. J. Nat. Prod. 2015, 78, 2310-2314. [CrossRef] [PubMed]

126. Ren, H.; Liu, W.-W. Nidurufin as a new cell cycle inhibitor from marine-derived fungus Penicillium flavidorsum SHK1-27. Arch. Pharm. Res. 2011, 34, 901-905. [CrossRef] [PubMed]

127. O'Malley, G.J.; Murphy, R.A., Jr.; Cava, M.P. Aflatoxin precursors: Total synthesis of (.+-.)-averufin and (.+-.)-nidurufin. J. Org. Chem. 1985, 50, 5533-5537. [CrossRef]

128. Liu, W.; Gu, Q.; Zhu, W.; Cui, C.; Fan, G. Two new benzoquinone derivatives and two new bisorbicillinoids were isolated from a marine-derived fungus Penicillium terrestre. J. Antibiot. 2005, 58, 441-446. [CrossRef] [PubMed]

129. Liu, W.; Gu, Q.; Zhu, W.; Cui, C.; Fan, G. Dihydrotrichodimerol and tetrahydrotrichodimerol, two new bisorbicillinoids, from a marine-derived Penicillium terrestre. J. Antibiot. 2005, 58, 621-624. [CrossRef] [PubMed]

130. Harned, A.M.; Volp, K.A. The sorbicillinoid family of natural products: Isolation, biosynthesis, and synthetic studies. Nat. Prod. Rep. 2011, 28, 1790-1810. [CrossRef] [PubMed]

131. Li, D.; Chen, L.; Zhu, T.; Kurtán, T.; Mándi, A.; Zhao, Z.; Li, J.; Gu, Q. Chloctanspirones A and B, novel chlorinated polyketides with an unprecedented skeleton, from marine sediment derived fungus Penicillium terrestre. Tetrahedron 2011, 67, 7913-7918. [CrossRef]

132. Janin, Y.L. Heat shock protein 90 inhibitors. A text book example of medicinal chemistry? J. Med. Chem. 2005, 48, 7503-7512. [CrossRef] [PubMed]

133. De Castro, M.V.; Ióca, L.P.; Williams, D.E.; Costa, B.Z.; Mizuno, C.M.; Santos, M.F.; de Jesus, K.; Ferreira, E.V.L.; Seleghim, M.H.; Sette, L.D. Condensation of Macrocyclic Polyketides Produced by Penicillium sp. DRF2 with Mercaptopyruvate Represents a New Fungal Detoxification Pathway. J. Nat. Prod. 2016, 79, 1668-1678. [CrossRef] [PubMed]

134. Greve, H.; Schupp, P.J.; Eguereva, E.; Kehraus, S.; Kelter, G.; Maier, A.; Fiebig, H.H.; König, G.M. Apralactone A and a New Stereochemical Class of Curvularins from the Marine Fungus Curvularia sp. Eur. J. Org. Chem. 2008, 30, 5085-5092. [CrossRef] [PubMed]

135. Getino, M.; Fernández-López, R.; Palencia-Gándara, C.; Campos-Gómez, J.; Sánchez-López, J.M.; Martínez, M.; Fernández, A.; de la Cruz, F. Tanzawaic acids, a chemically novel set of bacterial conjugation inhibitors. PLoS ONE 2016, 11, e0148098. [CrossRef] [PubMed]

136. Shin, H.J.; Pil, G.B.; Heo, S.-J.; Lee, H.-S.; Lee, J.S.; Lee, Y.-J.; Lee, J.; Won, H.S. Anti-inflammatory activity of tanzawaic acid derivatives from a marine-derived fungus Penicillium steckii 108YD142. Mar. Drugs 2016, 14, 14. [CrossRef] [PubMed]

137. Cardoso-Martínez, F.; José, M.; Díaz-Marrero, A.R.; Darias, J.; Cerella, C.; Diederich, M.; Cueto, M. Tanzawaic acids isolated from a marine-derived fungus of the genus Penicillium with cytotoxic activities. Org. Biomol. Chem. 2015, 13, 7248-7256. [CrossRef] [PubMed]

138. Sun, Y.; Ai, X.; Hou, J.; Ye, X.; Liu, R.; Shen, S.; Li, Z.; Lu, S. Integrated discovery of FOXO1-DNA stabilizers from marine natural products to restore chemosensitivity to anti-EGFR-based therapy for metastatic lung cancer. Mol. Biosyst. 2017, 13, 330-337. [CrossRef] [PubMed]

139. Shigemori, H.; Wakuri, S.; Yazawa, K.; Nakamura, T.; Sasaki, T.; Kobayashi, J.I. Fellutamides A and B, cytotoxic peptides from a marine fish-possessing fungus Penicillium fellutanum. Tetrahedron 1991, 47, 8529-8534. [CrossRef]

140. Wu, C.-J.; Li, C.-W.; Cui, C.-B. Seven new and two known lipopeptides as well as five known polyketides: The activated production of silent metabolites in a marine-derived fungus by chemical mutagenesis strategy using diethyl sulphate. Mar. Drugs 2014, 12, 1815-1838. [CrossRef] [PubMed]

141. Zhang, Z.; Guo, W.; He, X.; Che, Q.; Zhu, T.; Gu, Q.; Li, D. Peniphenylanes A-G from the Deep sea-Derived Fungus Penicillium fellutanum HDN14-323. Planta Med. 2016, 82, 872-876. [CrossRef] [PubMed] 
142. Chen, L.; Fang, Y.; Zhu, T.; Gu, Q.; Zhu, W. Gentisyl alcohol derivatives from the marine-derived fungus Penicillium terrestre. J. Nat. Prod. 2007, 71, 66-70. [CrossRef] [PubMed]

143. Wang, J.; Lu, Z.; Liu, P.; Wang, Y.; Li, J.; Hong, K.; Zhu, W. Cytotoxic Polyphenols from the Fungus Penicillium expansum 091006 Endogenous with the Mangrove Plant Excoecaria agallocha. Planta Med. 2012, 78, 1861-1866. [CrossRef] [PubMed]

144. Lu, Z.; Zhu, H.; Fu, P.; Wang, Y.; Zhang, Z.; Lin, H.; Liu, P.; Zhuang, Y.; Hong, K.; Zhu, W. Cytotoxic polyphenols from the marine-derived fungus Penicillium expansum. J. Nat. Prod. 2010, 73, 911-914. [CrossRef] [PubMed]

145. Yang, J.; Huang, R.; Qiu, S.X.; She, Z.; Lin, Y. A new isobenzofuranone from the mangrove endophytic fungus Penicillium sp.(ZH58). Nat. Prod. Rep. 2013, 27, 1902-1905. [CrossRef] [PubMed]

146. Kornprobst, J.-M.; La Barre, S. New Trends in Marine Natural Products. J. Oceanogr. Mar. Res. 2014, 2, 1000e109.

147. Zeng, Y.; Wang, H.; Kamdem, R.S.; Orfali, R.S.; Dai, H.; Makhloufi, G.; Janiak, C.; Liu, Z.; Proksch, P. A new cyclohexapeptide, penitropeptide and a new polyketide, penitropone from the endophytic fungus Penicillium tropicum. Tetrahedron Lett. 2016, 57, 2998-3001. [CrossRef]

148. Koul, M.; Singh, S. Penicillium spp.: Prolific producer for harnessing cytotoxic secondary metabolites. Anti-Cancer Drugs 2017, 28, 11-30. [CrossRef] [PubMed]

149. Brady, S.F.; Simmons, L.; Kim, J.H.; Schmidt, E.W. Metagenomic approaches to natural products from free-living and symbiotic organisms. Nat. Prod. Rep. 2009, 26, 1488-1503. [CrossRef] [PubMed]

(C) 2017 by the authors. Licensee MDPI, Basel, Switzerland. This article is an open access article distributed under the terms and conditions of the Creative Commons Attribution (CC BY) license (http:/ / creativecommons.org/licenses/by/4.0/). 